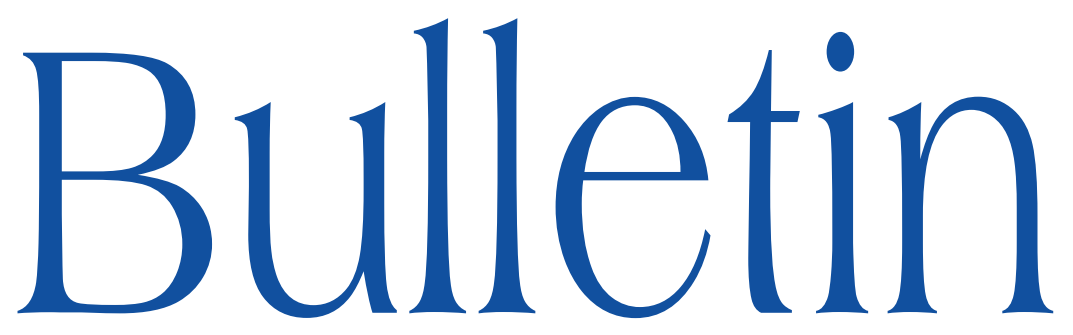

de la SOCIÉTÉ MATHÉMATIQUE DE FRANCE

\title{
LES THÉORÈMES DE LERAY ET DE FUJITA-KATO POUR LE SYSTÈME DE BOUSSINESQ PARTIELLEMENT VISQUEUX
}

Raphaël Danchin \& Marius Paicu

Tome 136

Fascicule 2

2008 


\title{
LES THÉORÈMES DE LERAY ET DE FUJITA-KATO POUR LE SYSTÈME DE BOUSSINESQ PARTIELLEMENT VISQUEUX
}

\author{
PAR RAPHAËL DANChIN \& MARIUS PAICU
}

\begin{abstract}
RÉSumÉ. - Dans cet article, on étudie le système de Boussinesq décrivant le phénomène de convection dans un fluide incompressible et visqueux. Ce système est composé des équations de Navier-Stokes incompressibles avec un terme de force verticale dont l'amplitude est transportée sans dissipation par le flot du champ de vitesses.

On montre que les résultats classiques pour le système de Navier-Stokes standard demeurent vrais pour le système de Boussinesq bien qu'il n'y ait pas d'amortissement sur le terme de force.

Plus précisément, on établit l'existence de solutions faibles globales d'énergie finie en n'importe quelle dimension et l'existence de solutions fortes uniques globales en dimension $N \geq 3$ pour de petites données initiales. Dans le cas particulier de la dimension deux, les solutions d'énergie finie sont uniques pour n'importe quelle donnée initiale dans $L^{2}\left(\mathbb{R}^{2}\right)$.
\end{abstract}

Texte reçu le 31 mai 2007, accepté le 17 juillet 2007

RAPhaËL Danchin, LAMA, UMR 8050, Université Paris-Est, 61 avenue du Général de Gaulle, 94010 Créteil Cedex, France • E-mail : danchin@univ-paris12.fr Marius Paicu, Laboratoire de Mathématiques, Université Paris 11, bât. 425, 91405 Orsay Cedex, France - E-mail : marius.paicu@math.u-psud.fr

Classification mathématique par sujets (2000). - 35Q35, 76N10, 35B65, 76D99.

Mots clefs. - Système de Boussinesq, solutions faibles, estimations à pertes, régularité critique. 
Abstract (The Leray and Fujita-Kato theorems for the Boussinesq system with partial viscosity)

We are concerned with the so-called Boussinesq equations with partial viscosity. These equations consist of the ordinary incompressible Navier-Stokes equations with a forcing term which is transported with no dissipation by the velocity field. Such equations are simplified models for geophysics (in which case the forcing term is proportional either to the temperature, or to the salinity or to the density).

In the present paper, we show that the standard theorems for incompressible NavierStokes equations may be extended to Boussinesq system despite the fact that there is no dissipation or decay at large time for the forcing term.

More precisely, we state the global existence of finite energy weak solutions in any dimension, and global well-posedness in dimension $N \geq 3$ for small data. In the twodimensional case, the finite energy global solutions are shown to be unique for any data in $L^{2}\left(\mathbb{R}^{2}\right)$.

\section{Introduction}

Dans cet article, on étudie l'évolution d'un fluide incompressible visqueux soumis à une force verticale. On suppose que l'amplitude $\theta$ de cette force est transportée par le flot du champ de vitesses $u$ du fluide. Le système de Boussinesq régissant l'évolution de $(\theta, u)$ s'écrit donc :

$$
\left\{\begin{array}{l}
\partial_{t} \theta+u \cdot \nabla \theta=0 \\
\partial_{t} u+u \cdot \nabla u-\nu \Delta u+\nabla \Pi=\theta e_{N} \\
\operatorname{div} u=0 .
\end{array}\right.
$$

Les inconnues $\theta, u$ et $\Pi$ dépendent du temps $t \geq 0$ et de la variable d'espace $x$. Pour s'abstraire des difficultés dues aux conditions aux limites, on suppose que le fluide remplit tout l'espace (donc $x$ décrit $\mathbb{R}^{N}$ tout entier).

Le système (1) est un modèle simplifié courant pour l'évolution de fluides géophysiques (voir par exemple [27] ou [29]). Le champ de vecteurs $u$ et le scalaire $\Pi$ désignent alors respectivement la vitesse et la pression du fluide considéré et $\theta$, une quantité scalaire ${ }^{(1)}$ transportée par le fluide.

Pour avoir un modèle plus réaliste, il conviendrait de rajouter des termes de forces extérieures aux deux équations. Nous les supposerons nuls pour simplifier la présentation. Notons au passage que dans le cas particulier où $\theta \equiv 0$, on retrouve le système de Navier-Stokes «classique ».

Les prémices de l'étude mathématique du système de Boussinesq sont relativement récentes et ne traduisent pas uniquement une préoccupation d'ordre

(1) Nous appellerons cette quantité « température » pour fixer les idées mais qui physiquement pourrait parfaitement être proportionnelle à la densité ou à la salinité.

TOME $136-2008-\mathrm{N}^{\mathrm{O}} 2$ 
physique. En réalité, l'engouement des mathématiciens pour ce modèle se limite essentiellement au cas de la dimension deux du fait d'une ressemblance formelle (dans le cas $\nu=0$ ) avec le système d'Euler incompressible axisymétrique avec swirl. On peut montrer que l'apparition de singularités au temps $T$ est liée à l'explosion simultanée de $\nabla \theta$ et du tourbillon dans $L^{1}\left(0, T ; L^{\infty}\right)$ (voir [16]). Malheureusement, déterminer si ces quantités explosent effectivement semble au moins aussi difficile que répondre au problème similaire pour le système d'Euler incompressible en dimension 3.

Dans le cas $\nu>0$ en revanche, les résultats obtenus sont nettement plus complets. Divers auteurs ont établi l'existence globale dans le cas $N=2$ lorsque l'équation sur $\theta$ comporte en plus un terme de diffusion (voir [30] et les références qui s'y trouvent).

L'existence globale en dimension deux sans condition de petitesse demeure valable dans la situation qui nous intéresse où $\theta$ est transporté sans diffusion. Le cas de données régulières dans des espaces de Sobolev a été résolu (indépendamment) par T. Hou et C. Li dans [20], et par D. Chae dans [5]. Très récemment, $\mathrm{H}$. Abidi et T. Hmidi ont établi dans [1] l'existence globale et unicité sans condition de petitesse pour des données initiales $\left(\theta_{0}, u_{0}\right)$ appartenant à un très gros sous-espace de $\left(L^{2}\left(\mathbb{R}^{2}\right)\right)^{3}$. Comme on peut par ailleurs construire des solutions faibles globales à la Leray pour des données initiales $\theta_{0}$ et $u_{0}$ dans $L^{2}\left(\mathbb{R}^{2}\right)$ (voir par exemple [19]), il est raisonnable de penser que ces solutions « faibles » sont uniques.

Dans cet article, nous souhaitons montrer que trois des résultats mathématiques les plus célèbres pour le système de Navier-Stokes incompressible sont encore valables pour le système de Boussinesq (1), à savoir :

- l'existence de solutions faibles globales d'énergie finie en n'importe quelle dimension (théorème de Leray, voir [24]),

- l'unicité des solutions d'énergie finie en dimension deux (résultat figurant implicitement dans un autre article de J. Leray, voir [23], et démontré par J.-L. Lions et G. Prodi dans [25]),

- l'existence de solutions globales uniques pour des données petites en dimension $N \geq 3$ (théorème de Fujita-Kato [17]).

Nous verrons plus loin que la démonstration de l'existence globale de solutions faibles se fait en suivant la démarche originale de J. Leray : la présence de la température nécessite un peu d'attention mais n'introduit pas de difficulté majeure.

En revanche, pour démontrer l'unicité en dimension deux, l'approche naïve consistant à écrire le système vérifié par la différence de deux solutions semble vouée à l'échec. En effet, du fait de la présence d'une équation de transport dans le système, les estimations de stabilité ne peuvent être établies qu'avec perte 
d'au moins une dérivée donc dans un espace à régularité strictement négative. Or, pour contrôler ce type de régularité, l'hypothèse que le champ de vitesses soit au moins lipschitzien semble inévitable. Mais le champ de vitesses construit n'est a priori que dans $L_{l o c}^{2}\left(\mathbb{R}_{+} ; H^{1}\right)$.

Enfin, pour établir l'existence de solutions fortes globales en dimension $N \geq$ 3, une application directe de l'approche de Fujita-Kato associant point fixe contractant et utilisation du semi-groupe de la chaleur ne saurait convenir. En effet, la méthode de Fujita-Kato exige non seulement une donnée initiale petite mais aussi un terme de force $f$ qui soit intégrable en temps sur $\mathbb{R}_{+}$entier, et d'intégrale petite. À moins que $\theta$ ne soit identiquement nulle, cette dernière condition n'est pas vérifiée par le terme de force $\theta e_{N}$ puisque $\theta$ est transportée sans amortissement donc est constante le long des caractéristiques.

Nous avons adopté le plan suivant. La première section est consacrée à la présentation de nos résultats principaux. Dans la partie suivante, nous démontrons l'analogue du théorème de Leray pour le système de Boussinesq à l'aide d'arguments élémentaires d'analyse fonctionnelle. Dans la troisième section, nous présentons l'artillerie technique nécessaire pour venir à bout du reste de notre programme : espaces de Lorentz et de Besov, décomposition de LittlewoodPaley et calcul paradifférentiel. La quatrième partie regroupe diverses estimations a priori - dont certaines avec perte de régularité - pour l'équation de la chaleur ou de Stokes, l'équation de transport et le système de Navier-Stokes. Les résultats liés aux solutions fortes sont alors démontrés dans la cinquième partie, et l'unicité des solutions faibles d'énergie finie en dimension deux, dans la sixième partie. Nous avons regroupé en dernière section quelques résultats supplémentaires. Un lemme technique figure en appendice.

\section{Résultats principaux}

Avant d'énoncer notre résultat d'existence globale de solutions faibles, présentons brièvement les égalités d'énergie formelles associées au système.

Soit donc $(\theta, u, \nabla \Pi)$ une solution régulière et décroissante à l'infini du système de Boussinesq avec données $\left(\theta_{0}, u_{0}\right)$. Tout d'abord, sachant que le champ de vitesses est à divergence nulle, on a

$$
\|\theta(t)\|_{L^{p}}=\left\|\theta_{0}\right\|_{L^{p}} \text { pour tout } t \geq 0 \text { et } 1 \leq p \leq \infty .
$$

Par ailleurs, en prenant le produit scalaire $L^{2}\left(\mathbb{R}^{N}\right)^{N}$ de l'équation de la vitesse avec $u$ puis en intégrant en temps, on obtient

$$
\|u(t)\|_{L^{2}}^{2}+2 \nu \int_{0}^{t}\|\nabla u\|_{L^{2}}^{2} d \tau=\left\|u_{0}\right\|_{L^{2}}^{2}+2 \int_{0}^{t} \int_{\mathbb{R}^{N}} \theta u_{N} d x d \tau .
$$

TOME $136-2008-\mathrm{N}^{\mathrm{O}} 2$ 
Il est clair que l'intégrale du terme de droite peut être absorbée par le membre de gauche pourvu que l'on dispose d'un contrôle sur la norme $H^{-1}$ de $\theta$. Ce contrôle est donné par (2) dès que $L^{p}\left(\mathbb{R}^{N}\right)$ s'injecte continûment dans $H^{-1}\left(\mathbb{R}^{N}\right)$. Si $N \geq 3$ (resp. $N=2$ ), cette condition est vérifiée si et seulement si $p \geq \frac{2 N}{N+2}$ (resp. $\left.p>1\right)$.

Comme de coutume, l'obtention des solutions faibles globales se fera en passant à la limite dans une suite de solutions approchées vérifiant (2) et (3). Le passage à la limite dans le terme $u \cdot \nabla \theta=\operatorname{div}(u \theta)$ exige que l'injection de $L^{p}\left(\mathbb{R}^{N}\right)$ dans $H^{-1}\left(\mathbb{R}^{N}\right)$ soit localement compacte, donc que $p>\frac{2 N}{N+2}$.

Ces considérations sommaires mènent à l'énoncé suivant :

THÉORÈme 1.1. - Soit $\theta_{0} \in L^{p}\left(\mathbb{R}^{N}\right)$ avec $\frac{2 N}{N+2}<p \leq 2$, et $u_{0}$ un champ de vitesses à divergence nulle et coefficients dans $L^{2}\left(\mathbb{R}^{N}\right)$. Alors le système de Boussinesq avec donnée initiale $\left(\theta_{0}, u_{0}\right)$ admet une solution faible globale

$$
(\theta, u) \in L^{\infty}\left(\mathbb{R}_{+} ; L^{p}\left(\mathbb{R}^{N}\right)\right) \times\left(L_{l o c}^{\infty}\left(\mathbb{R}_{+} ; L^{2}\left(\mathbb{R}^{N}\right)\right) \cap L_{l o c}^{2}\left(\mathbb{R}_{+} ; H^{1}\left(\mathbb{R}^{N}\right)\right)\right)^{N},
$$

telle que pour tout $t \geq 0$, on ait

$$
\|\theta(t)\|_{L^{p}} \leq\left\|\theta_{0}\right\|_{L^{p}}
$$

et

$$
\|u(t)\|_{L^{2}}^{2}+\nu \int_{0}^{t}\|\nabla u\|_{L^{2}}^{2} d \tau \leq C\left(\left\|u_{0}\right\|_{L^{2}}^{2}+\nu^{\alpha-1} t^{\alpha+1}\left\|\theta_{0}\right\|_{L^{p}}^{2}\right)
$$

pour une constante $C$ ne dépendant que de $N$ et de $p$, et $\alpha=1-N\left(\frac{1}{p}-\frac{1}{2}\right)$.

Dans le cas $\theta \equiv 0$, il est bien connu que les solutions faibles globales en dimension deux sont uniques (voir par exemple [25]). Il est donc légitime de chercher à démontrer que cette propriété persiste pour le système de Boussinesq avec des données quelconques dans $L^{2}\left(\mathbb{R}^{2}\right)$. À notre connaissance, le résultat le plus proche allant dans ce sens a été établi par H. Abidi et T. Hmidi dans [1] (voir aussi [19]) : si $\theta_{0}$ appartient à l'espace de Besov homogène ${ }^{(2)} \dot{B}_{2,1}^{0}\left(\mathbb{R}^{2}\right)$ et $u_{0}$ est dans $\dot{B}_{\infty, 1}^{-1} \cap L^{2}\left(\mathbb{R}^{2}\right)$ alors il y a unicité de la solution globale construite.

Nous démontrons ici qu'il y a encore unicité des solutions faibles d'énergie finie en dimension deux sous la seule hypothèse que les données sont dans $L^{2}\left(\mathbb{R}^{2}\right)$.

Cette propriété remarquable découle du fait que le champ de vitesses construit appartient aussi à l'espace $\widetilde{L}_{\text {loc }}^{1}\left(\mathbb{R}_{+} ; H^{2}\left(\mathbb{R}^{2}\right)\right.$ ) (défini plus loin en (18)) qui est à peine plus gros que $L_{l o c}^{1}\left(\mathbb{R}_{+} ; H^{2}\left(\mathbb{R}^{2}\right)\right)$. De ce fait, le champ de vitesses est presque localement intégrable en temps à valeurs quasi-lipschitziennes et l'on peut faire appel à des estimations avec perte de régularité dans l'esprit de celles qui ont été établies dans [2] ou, plus récemment, dans [13].

(2) Voir la définition 3.3. 
ThÉORÈme 1.2. - Supposons $N=2$. Soit $\left(\theta_{0}, u_{0}\right) \in L^{2}\left(\mathbb{R}^{2}\right)^{3}$ avec $u_{0} \grave{a}$ divergence nulle. Alors le système (1) a une unique solution globale $(\theta, u, \nabla \Pi)$ telle que

$$
\theta \in \mathcal{C}\left(\mathbb{R}_{+} ; L^{2}\right) \quad \text { et } \quad u \in \mathcal{C}\left(\mathbb{R}_{+} ; L^{2}\right) \cap L_{\text {loc }}^{2}\left(\mathbb{R}_{+} ; H^{1}\right) .
$$

De plus la norme $L^{2}$ de $\theta$ est conservée au cours de l'évolution, l'égalité (3) est satisfaite et $u$ appartient à l'espace $\widetilde{L}_{\text {loc }}^{1}\left(\mathbb{R}_{+} ; H^{2}\right)$ défini au-dessus de la remarque 3.12 .

La dernière partie de notre programme consiste à établir un résultat d'existence globale de solutions fortes à données petites en dimension $N \geq 3$, dans l'esprit de celui de Fujita-Kato pour le système de Navier-Stokes incompressible (voir [17]).

Rappelons que le théorème de Fujita-Kato se démontre en récrivant le système de Navier-Stokes en termes de problème de point fixe pour une fonctionnelle construite à l'aide du semi-groupe de Stokes. Sous des hypothèses de petitesse adéquates, le théorème du point fixe de Picard permet alors d'obtenir une solution globale unique. Cette approche s'avère particulièrement performante si l'espace fonctionnel $F$ utilisé est critique, i.e. respecte l'invariance par changement d'échelle (ou scaling en anglais) du système de Navier-Stokes. Cela amène à choisir pour $F$ un espace de distributions sur $\mathbb{R}^{+} \times \mathbb{R}^{N}$ à norme invariante pour tout $\lambda>0$ par la transformation

$$
u(t, x) \longmapsto \lambda u\left(\lambda^{2} t, \lambda x\right)
$$

et donc à choisir une vitesse initiale $u_{0}$ dans un espace fonctionnel $E$ à norme invariante par la transformation $u_{0} \mapsto \lambda u_{0}(\lambda \cdot)$. H. Fujita et T. Kato démontrent ainsi que le système de Navier-Stokes est globalement bien posé pour des données initiales petites par rapport à la viscosité dans l'espace de Sobolev homogène $\dot{H}^{\frac{1}{2}}\left(\mathbb{R}^{3}\right)$ (voir [17] et [7]). Le résultat de Fujita-Kato se généralise à un grand nombre d'espaces critiques (voir e.g. [22] ou [26]).

Un calcul facile montre que le système de Boussinesq est invariant par la transformation

$$
u(t, x) \longmapsto \lambda u\left(\lambda^{2} t, \lambda x\right) \text { et } \theta(t, x) \longmapsto \lambda^{3} \theta\left(\lambda^{2} t, \lambda x\right) .
$$

Autrement dit, les espaces critiques pour la vitesse sont les mêmes que pour le système de Navier-Stokes et il faut en quelque sorte exiger deux dérivées de moins sur la température.

En l'absence de dissipation sur la température, il ne semble pas possible de démontrer que le système de Boussinesq est bien posé même localement pour des données aussi peu régulières. On peut cependant établir l'existence locale pour des données initiales dans l'espace de Besov homogène $\dot{B}_{N, 1}^{0}\left(\mathbb{R}^{N}\right)$ (gros sous-espace de $L^{N}\left(\mathbb{R}^{N}\right)$ ) qui est critique pour la vitesse seulement : 
ThÉORÈme 1.3. - Supposons $N \geq 2$. Soit $\theta_{0} \in \dot{B}_{N, 1}^{0}$ et $u_{0}$ un champ de vecteurs à divergence nulle et coefficients dans $\dot{B}_{N, 1}^{0}$. Alors le système de Boussinesq admet une unique solution locale $(\theta, u, \nabla \Pi)$ dans l'espace

$E_{T}:=\mathcal{C}\left([0, T] ; \dot{B}_{N, 1}^{0}\right) \times\left(\mathcal{C}\left([0, T] ; \dot{B}_{N, 1}^{0}\right) \cap L^{1}\left(0, T ; \dot{B}_{N, 1}^{2}\right)\right)^{N} \times\left(L^{1}\left(0, T ; \dot{B}_{N, 1}^{0}\right)\right)^{N}$.

Si de plus $\theta \in L^{\infty}\left(0, T^{*} ; \dot{B}_{N, 1}^{0}\right)$ et $u \in L^{\infty}\left(0, T^{*} ; \dot{B}_{N, 1}^{0}\right) \cap L^{1}\left(0, T^{*} ; \dot{B}_{N, 1}^{2}\right)$ alors la solution peut être prolongée au-delà de $T^{*}$.

Si la construction de solutions à temps petit peut se faire par des arguments standard, montrer l'existence de solutions fortes globales est nettement plus délicat. En effet, comme expliqué dans l'introduction, l'absence d'amortissement pour la température interdit l'approche classique associant estimations pour le semi-groupe de la chaleur et point fixe de Picard, et le scaling de la température semble bien trop bas pour pouvoir travailler directement dans des espaces invariants par changement d'échelle.

Notre stratégie est la suivante : trouver deux espaces fonctionnels, $X$ pour la vitesse initiale $u_{0}$ et $Y$ pour la température initiale $\theta_{0}$ tels que, en notant $\mathcal{P}$ le projecteur orthogonal sur les champs à divergence nulle et $\left(e^{\tau \Delta}\right)_{\tau>0}$ le semi-groupe de la chaleur, on ait les estimations suivantes :

$$
\begin{gathered}
\|\theta\|_{L^{\infty}(0, T ; Y)} \leq\left\|\theta_{0}\right\|_{Y}, \quad\left\|e^{t \Delta} u_{0}\right\|_{L^{\infty}(0, T ; X)} \leq\left\|u_{0}\right\|_{X}, \\
\left\|\int_{0}^{t} e^{(t-s) \Delta} \mathcal{P}\left(\theta e_{N}\right) d s\right\|_{L^{\infty}(0, T ; X)} \leq C_{1}\|\theta\|_{L^{\infty}(0, T ; Y)}, \\
\left\|\int_{0}^{t} e^{(t-s) \Delta} \mathcal{P} \operatorname{div}(u \otimes u)(s) d s\right\|_{L^{\infty}(0, T ; X)} \leq C_{2}\|u\|_{L^{\infty}(0, T ; X)}^{2}
\end{gathered}
$$

avec des constantes $C_{1}$ et $C_{2}$ indépendantes du temps $T$. (Nous avons supposé que $\nu=1$ pour simplifier l'heuristique.)

Pour de tels espaces, on obtiendra alors

$$
\|u\|_{L^{\infty}(0, T ; X)} \leq\left\|u_{0}\right\|_{X}+C_{1}\left\|\theta_{0}\right\|_{Y}+C_{2}\|u\|_{L^{\infty}(0, T ; X)}^{2} .
$$

Supposons que (1) admette une solution locale $(\theta, u)$ dans $L^{\infty}([0, T] ; Y) \times$ $L^{\infty}([0, T] ; X)$ et que de plus $C_{1}\left\|\theta_{0}\right\|_{Y}+\left\|u_{0}\right\|_{X} \leq c$ avec $c$ suffisamment petit. Soit $T^{*}$ le temps maximal pour lequel $\|u\|_{L^{\infty}\left(0, T^{*} ; X\right)} \leq 2 c$. Si l'on a choisi $c$ de telle sorte que $4 c C_{2}<1$, l'inégalité ci-dessus implique que $\|u\|_{L^{\infty}\left(0, T^{*} ; X\right)}<2 c$. Par des arguments classiques de bootstrap, on conclut alors que $T^{*}=+\infty$.

Nous verrons plus loin que l'on peut choisir pour $X$ l'espace de Lorentz $L^{N, \infty}\left(\mathbb{R}^{N}\right)$, et pour $Y$ l'espace de Lebesque $L^{\frac{N}{3}}\left(\mathbb{R}^{N}\right)$ (voire $L^{\frac{N}{3}, \infty}\left(\mathbb{R}^{N}\right)$ si $N \geq$ 4). Notons au passage que ces espaces sont critiques. 
À moins que $\theta \equiv 0$, il n'est pas clair que l'on puisse démontrer l'existence et l'unicité de solutions dans ces espaces sans hypothèse supplémentaire de régularité. Pour y remédier, nous allons devoir exiger plus de régularité sur les données initiales. Cette régularité doit de plus pouvoir être transportée globalement si les données sont petites dans $X \times Y$. Le caractère global demande un peu d'attention car les estimations habituelles de régularité sur les solutions d'une équation de transport font apparaître un terme qui dépend exponentiellement de la norme lipschitzienne du champ de vitesse. On sait cependant depuis un article de M. Vishik que ces estimations sont «meilleures» dans les espaces de Besov d'indice nul (voir [31] et la proposition 4.8 pour plus de détails).

Ces considérations mènent au résultat suivant d'existence globale à données petites :

ThÉorème 1.4. - Supposons que $N \geq 3$. Soit $\theta_{0} \in \dot{B}_{N, 1}^{0}\left(\mathbb{R}^{N}\right)$ et $u_{0} \in$ $\dot{B}_{p, 1}^{-1+\frac{N}{p}}\left(\mathbb{R}^{N}\right)$ pour un $p \in[N, \infty]$. Il existe une constante $c>0$ ne dépendant que de $N$ telle que si de plus $u_{0} \in L^{N, \infty}, \theta_{0} \in L^{\frac{N}{3}}$ et

$$
\left\|u_{0}\right\|_{L^{N, \infty}}+\nu^{-1}\left\|\theta_{0}\right\|_{L^{\frac{N}{3}}} \leq c \nu
$$

alors le système de Boussinesq admet une unique solution globale

$$
\begin{aligned}
(\theta, u, \nabla \Pi) \in \mathcal{C}\left(\mathbb{R}_{+} ; \dot{B}_{N, 1}^{0}\right) & \times\left(\mathcal{C}\left(\mathbb{R}_{+} ; \dot{B}_{p, 1}^{\frac{N}{p}-1}\right) \cap L_{l o c}^{1}\left(\mathbb{R}_{+} ; \dot{B}_{p, 1}^{\frac{N}{p}+1}\right)\right)^{N} \\
& \times\left(L_{l o c}^{1}\left(\mathbb{R}_{+} ; \dot{B}_{p, 1}^{\frac{N}{p}-1}\right)\right)^{N} .
\end{aligned}
$$

De plus, il existe une constante $C$ ne dépendant que de $N$ telle que pour tout temps $t \geq 0$, on ait

$$
\begin{gathered}
\|\theta(t)\|_{L^{\frac{N}{3}}}=\left\|\theta_{0}\right\|_{L^{\frac{N}{3}}}, \quad\|u(t)\|_{L^{N, \infty}} \leq C\left(\left\|u_{0}\right\|_{L^{N, \infty}}+\nu^{-1}\left\|\theta_{0}\right\|_{L^{\frac{N}{3}}}\right) \\
\|u\|_{L_{t}^{\infty}\left(\dot{B}_{p, 1}^{\frac{N}{p}-1}\right)}+\nu\|u\|_{L_{t}^{1}\left(\dot{B}_{p, 1}^{\frac{N}{p}+1}\right)} \leq C\left\|u_{0}\right\|_{\dot{B}_{p, 1}^{\frac{N}{p}-1}} e^{C t \nu^{-1}\left\|\theta_{0}\right\|_{\dot{B}_{N, 1}^{0}}}+\nu\left(e^{C t \nu^{-1}\left\|\theta_{0}\right\|_{\dot{B}_{N, 1}^{0}}^{0}-1}\right), \\
\|\theta(t)\|_{\dot{B}_{N, 1}^{0}} \leq C\left\|\theta_{0}\right\|_{\dot{B}_{N, 1}^{0}} e^{C t \nu^{-1}\left\|\theta_{0}\right\|_{\dot{B}_{N, 1}^{0}}}\left(1+C \nu^{-1}\left\|u_{0}\right\|_{\dot{B}_{N, 1}^{0}}\right) .
\end{gathered}
$$

Remarque 1.5. - À partir de la dimension quatre, l'espace $L^{\frac{N}{3}}\left(\mathbb{R}^{N}\right)$ peut être remplacé par $L^{\frac{N}{3}, \infty}\left(\mathbb{R}^{N}\right)$.

Remarque 1.6. - Notons que les hypothèses supplémentaires sur la vitesse sont critiques en terme de scaling. Par ailleurs, l'appartenance de $\theta_{0}$ à l'espace de Besov homogène $\dot{B}_{N, 1}^{0}$ (qui est un sous-espace strict de l'espace de Besov non homogène $\left.B_{N, 1}^{0}\right)$ est assurée par $\theta_{0} \in L^{\frac{N}{3}, \infty} \cap B_{N, 1}^{0}$. De même, si $p>N$,

TOME $136-2008-\mathrm{N}^{\mathrm{O}} 2$ 
l'hypothèse $u_{0} \in \dot{B}_{p, 1}^{-1+\frac{N}{p}}$ est garantie par $u_{0} \in L^{N, \infty} \cap B_{p, 1}^{-1+\frac{N}{p}}$ (voir le lemme $3.9)$.

Notation. - Dans tout l'article, $C$ désigne une « constante » susceptible de changer de ligne en ligne et dont la valeur exacte n'influe pas sur l'exactitude des calculs. On utilise parfois la notation abrégée $A \lesssim B$ au lieu de $A \leq C B$.

\section{Solutions faibles globales}

Cette section est consacrée à la démonstration du théorème 1.1.

Fixons une fonction $\chi \in \mathcal{C}_{c}^{\infty}\left(\mathbb{R}^{N}\right)$ positive et d'intégrale 1 , et notons $I_{r}$ $(r>0)$ l'opérateur de convolution par la fonction $r^{-N} \chi\left(r^{-1} \cdot\right)$. Dans un premier temps, nous allons résoudre le système de Boussinesq régularisé suivant :

$$
\left\{\begin{array}{l}
\partial_{t} \theta+I_{r} u \cdot \nabla \theta=0, \\
\partial_{t} u+\mathcal{P}\left(I_{r} u \cdot \nabla u\right)-\nu \Delta u=\mathcal{P}\left(I_{r} \theta e_{N}\right),
\end{array}\right.
$$

avec données initiales $\theta_{0}$ et $u_{0}$ dans $L^{2}\left(\mathbb{R}^{N}\right)$.

Proposition 2.1. - Pour tout couple de données initiales $\left(\theta_{0}, u_{0}\right)$ dans $L^{2}\left(\mathbb{R}^{N}\right)$ tel que div $u_{0}=0$, le système (5) admet une solution faible globale $(\theta, u)$ telle que

$$
\theta \in \mathcal{C}\left(\mathbb{R}_{+} ; L^{2}\right), \quad u \in L_{l o c}^{\infty}\left(\mathbb{R}_{+} ; L^{2}\right) \cap L_{l o c}^{2}\left(\mathbb{R}_{+} ; H^{1}\right)
$$

et vérifiant l'inégalité d'énergie suivante pour tout $t \in \mathbb{R}^{+}$:

$$
\|u(t)\|_{L^{2}}^{2}+2 \nu \int_{0}^{t}\|\nabla u\|_{L^{2}}^{2} d \tau \leq\left\|u_{0}\right\|_{L^{2}}^{2}+2 \int_{0}^{t} \int_{\mathbb{R}^{N}} I_{r} \theta u_{N} d x d \tau .
$$

Preuve. - Nous allons résoudre le système (5) à l'aide de la méthode de Friedrichs. Pour cela, on définit l'opérateur de troncature spectrale $J_{n}$ par

$$
\widehat{J_{n} f}(\xi)=1_{\left[\frac{1}{n}, n\right]}(|\xi|) \widehat{f}(\xi)
$$

et l'on cherche à résoudre le système suivant pour $n \geq 1$ :

$$
\left\{\begin{array}{l}
\partial_{t} \theta^{n}+J_{n}\left(I_{r} \mathcal{P} J_{n} u^{n} \cdot \nabla J_{n} \theta^{n}\right)=0 \\
\partial_{t} u^{n}+\mathcal{P} J_{n}\left(I_{r} \mathcal{P} J_{n} u^{n} \cdot \nabla \mathcal{P} J_{n} u^{n}\right)-\nu \Delta \mathcal{P} J_{n} u^{n}=\mathcal{P}\left(I_{r} J_{n} \theta^{n} e_{N}\right) \\
\left(\theta^{n}, u^{n}\right)_{\mid t=0}=\left(J_{n} \theta_{0}, J_{n} u_{0}\right)
\end{array}\right.
$$

Ce système est une équation différentielle ordinaire sur $L^{2}$ vérifiant les hypothèses du théorème de Cauchy-Lipschitz. Il admet donc une unique solution maximale $\left(\theta^{n}, u^{n}\right) \in \mathcal{C}^{1}\left(\left[0, T_{n}\left[;\left(L^{2}\left(\mathbb{R}^{N}\right)\right)^{N+1}\right)\right.\right.$ pour un $T_{n}>0$. 
Comme de plus $\left(\mathcal{P} J_{n}\right)^{2}=\mathcal{P} J_{n}$ et $J_{n}^{2}=J_{n}$, le couple $\left(J_{n} \theta^{n}, \mathcal{P} J_{n} u^{n}\right)$ est aussi solution de (7). Par unicité, on a donc $J_{n} \theta^{n}=\theta^{n}$ et $\mathcal{P} J_{n} u^{n}=u^{n}$. Cela entraîne que $\theta^{n}$ et $u^{n}$ sont dans $\mathcal{C}^{1}\left(\left[0, T^{n}\left[; H^{\infty}\right)\right.\right.$ et vérifient

$$
\left\{\begin{array}{l}
\partial_{t} \theta^{n}+J_{n}\left(I_{r} u^{n} \cdot \nabla \theta^{n}\right)=0, \\
\partial_{t} u^{n}+\mathcal{P} J_{n}\left(I_{r} u^{n} \cdot \nabla u^{n}\right)-\nu \Delta u^{n}=\mathcal{P}\left(I_{r} \theta^{n} e_{N}\right) .
\end{array}\right.
$$

Une méthode d'énergie élémentaire assure alors que $\left\|\theta^{n}(t)\right\|_{L^{2}}=\left\|J_{n} \theta_{0}\right\|_{L^{2}}$ pour tout $t \in \mathbb{R}_{+}$, et

$$
\left\|u^{n}(t)\right\|_{L^{2}}^{2}+2 \nu \int_{0}^{t}\left\|\nabla u^{n}\right\|_{L^{2}}^{2} d \tau=\left\|J_{n} u_{0}\right\|_{L^{2}}^{2}+2 \int_{0}^{t} \int_{\mathbb{R}^{N}} I_{r} \theta^{n} u_{N}^{n} d x d \tau .
$$

Notons que ces deux égalités impliquent que $T_{n}=+\infty$. En effet, si $T_{n}$ est fini, on montre facilement en majorant le terme de droite de (9) à l'aide de l'inégalité de Cauchy-Schwarz, que $\left(\theta^{n}, u^{n}\right)$ est dans $L^{\infty}\left(0, T^{n} ; L^{2}\right)$ et peut donc être prolongée en vertu des théorèmes classiques sur les équations différentielles ordinaires.

Il s'agit maintenant de passer à la limite dans (8). Tout d'abord, il est clair que la suite $\left(\theta^{n}\right)_{n \in \mathbb{N}}$ est bornée dans $L^{\infty}\left(\mathbb{R}_{+} ; L^{2}\right)$ et que (9) assure que $\left(u^{n}\right)_{n \in \mathbb{N}}$ est bornée dans $L_{\text {loc }}^{\infty}\left(\mathbb{R}_{+} ; L^{2}\right) \cap L_{l o c}^{2}\left(\mathbb{R}_{+} ; H^{1}\right)$. En utilisant le système (8) et en remarquant que la suite $\left(I_{r} u^{n}\right)_{n \in \mathbb{N}}$ est bornée dans tous les espaces $L_{l o c}^{\infty}\left(\mathbb{R}_{+} ; H^{s}\right)$, on en déduit alors que $\left(\partial_{t} \theta^{n}\right)_{n \in \mathbb{N}}$ et $\left(\partial_{t} u^{n}\right)_{n \in \mathbb{N}}$ sont bornées dans $L_{l o c}^{2}\left(\mathbb{R}_{+} ; H^{-1}\right)$. Par des arguments de convergence faible et de compacité classiques, on conclut qu'il existe $\theta \in L^{\infty}\left(\mathbb{R}_{+} ; L^{2}\right)$ et $u \in L_{\text {loc }}^{\infty}\left(\mathbb{R}_{+} ; L^{2}\right) \cap L_{\text {loc }}^{2}\left(\mathbb{R}_{+} ; H^{1}\right)$ à divergence nulle, tels que, à extraction près,

- $\theta^{n}$ tend vers $\theta$ dans $L_{\text {loc }}^{\infty}\left(\mathbb{R}_{+} ; H_{\text {loc }}^{-\eta}\right)$ pour tout $\eta>0$,

- $\theta^{n}(t)$ tend faiblement dans $L^{2}$ vers $\theta(t)$ pour tout $t \in \mathbb{R}_{+}$(et donc $I_{r} \theta^{n}(t)$ tend fortement vers $I_{r} \theta(t)$ dans $\left.L^{2}\right)$,

- $u^{n}$ tend vers $u$ dans $L_{\text {loc }}^{2}\left(\mathbb{R}_{+} ; H_{\text {loc }}^{1-\varepsilon}\right)$ pour tout $\varepsilon>0$,

- $u^{n}(t)$ tend faiblement dans $L^{2}$ vers $u(t)$ pour tout $t \in \mathbb{R}_{+}$.

Ces propriétés de convergence permettent de passer à la limite dans le système (8) et de montrer que

$$
\lim _{n \rightarrow \infty} \int_{0}^{t} \int_{\mathbb{R}^{N}} I_{r} \theta^{n} u_{N}^{n} d x d \tau=\int_{0}^{t} \int_{\mathbb{R}^{N}} I_{r} \theta u_{N} d x d \tau .
$$

Le couple $(\theta, u)$ est donc une solution globale de (5) vérifiant (6).

Nous pouvons maintenant passer à la démonstration du théorème 1.1. Nous supposons donc que la température initiale $\theta_{0}$ appartient à $L^{p}\left(\mathbb{R}^{N}\right)$ avec $\frac{2 N}{N+2}<$ $p \leq 2$ et que $u_{0} \in L^{2}\left(\mathbb{R}^{N}\right)$ avec $\operatorname{div} u_{0}=0$. Il est clair que $I_{r} \theta_{0} \in L^{2}$ pour tout 
$r>0$. La proposition précédente appliquée avec $r=2^{-k}$ assure donc l'existence d'une solution globale $\left(\theta_{k}, u_{k}\right)$ vérifiant (6) pour le système approché

$$
\left\{\begin{array}{l}
\partial_{t} \theta_{k}+\operatorname{div}\left(I_{2^{-k}} u_{k} \theta_{k}\right)=0, \\
\partial_{t} u_{k}+\mathcal{P} \operatorname{div}\left(I_{2^{-k}} u_{k} \otimes u_{k}\right)-\nu \Delta u_{k}=\mathcal{P}\left(I_{2^{-k}} \theta_{k} e_{N}\right), \\
\left(\theta_{k}, u_{k}\right)_{\mid t=0}=\left(I_{2^{-k}} \theta_{0}, u_{0}\right) .
\end{array}\right.
$$

On constate que $\theta_{k}$ est solution d'une équation de transport par le champ de vecteurs régulier à divergence nulle $I_{2^{-k}} u_{k}$. Cela assure que $\theta_{k} \in \mathcal{C}\left(\mathbb{R}_{+} ; L^{2} \cap L^{p}\right)$ et que

$$
\left\|\theta_{k}(t)\right\|_{L^{p}}=\left\|I_{2^{-k}} \theta_{0}\right\|_{L^{p}} \leq\left\|\theta_{0}\right\|_{L^{p}} \text { pour tout } t \geq 0 .
$$

Ensuite, en vertu de (6) et de l'inégalité de Hölder, on a

$$
\left\|u_{k}(t)\right\|_{L^{2}}^{2}+2 \nu \int_{0}^{t}\left\|\nabla u_{k}\right\|_{L^{2}}^{2} d t \leq\left\|u_{0}\right\|_{L^{2}}^{2}+2 \int_{0}^{t}\left\|\theta_{k}\right\|_{L^{p}}\left\|u_{k}\right\|_{L^{p^{\prime}}} d t .
$$

Comme $\frac{2 N}{N+2}<p \leq 2$, on dispose de l'inégalité de Gagliardo-Nirenberg suivante :

$$
\left\|u_{k}\right\|_{L^{p^{\prime}}} \leq C\left\|u_{k}\right\|_{L^{2}}^{\alpha}\left\|\nabla u_{k}\right\|_{L^{2}}^{1-\alpha} \quad \text { avec } \quad \alpha=1-N\left(\frac{1}{p}-\frac{1}{2}\right) .
$$

En injectant cette inégalité dans (12) et en utilisant (11) et l'inégalité de Young, on trouve

$$
\left\|u_{k}(t)\right\|_{L^{2}}^{2}+\nu \int_{0}^{t}\left\|\nabla u_{k}\right\|_{L^{2}}^{2} d t \leq\left\|u_{0}\right\|_{L^{2}}^{2}+C \nu^{\frac{\alpha-1}{\alpha+1}}\left\|\theta_{0}\right\|_{L^{p}}^{\frac{2}{1+\alpha}} \int_{0}^{t}\left\|u_{k}\right\|_{L^{2}}^{\frac{2 \alpha}{1+\alpha}} d \tau,
$$

d'où, après une nouvelle utilisation de l'inégalité de Young,

$$
\left\|u_{k}(t)\right\|_{L^{2}}^{2}+\nu \int_{0}^{t}\left\|\nabla u_{k}\right\|_{L^{2}}^{2} d t \leq C\left(\left\|u_{0}\right\|_{L^{2}}^{2}+t^{\alpha+1} \nu^{\alpha-1}\left\|\theta_{0}\right\|_{L^{p}}^{2}\right) .
$$

Il reste à justifier le passage à la limite dans (10). Tout d'abord (11) et (13) assurent que $\left(\theta_{k}\right)_{k \in \mathbb{N}}$ est bornée dans $L^{\infty}\left(\mathbb{R}_{+} ; L^{p}\right)$ et que $\left(u_{k}\right)_{k \in \mathbb{N}}$ est bornée dans $L^{\infty}\left(\mathbb{R}_{+} ; L^{2}\right) \cap L^{2}\left(\mathbb{R}_{+} ; H^{1}\right)$. En utilisant $(7)$, on peut alors montrer que $\left(\partial_{t} \theta_{k}\right)_{k \in \mathbb{N}}$ et $\left(\partial_{t} u_{k}\right)_{k \in \mathbb{N}}$ sont bornées dans $L_{l o c}^{2}\left(\mathbb{R}_{+} ; H^{-\frac{N}{2}-1}\right)$. Par des arguments de compacité classiques et en notant que $L^{p}\left(\mathbb{R}^{N}\right) \hookrightarrow H^{-N\left(\frac{1}{p}-\frac{1}{2}\right)}\left(\mathbb{R}^{N}\right)$ pour $1<p \leq 2$, on en déduit, quitte à extraire, que :

- $\theta_{k}$ tend vers une fonction $\theta$ dans $L_{l o c}^{\infty}\left(\mathbb{R}_{+} ; H_{l o c}^{-N\left(\frac{1}{p}-\frac{1}{2}\right)-\eta}\right)$ pour tout $\eta>0$,

- $u_{k}$ tend vers un champ $u$ à divergence nulle dans $L_{l o c}^{2}\left(\mathbb{R}_{+} ; H_{l o c}^{1-\varepsilon}\right)$ pour tout $\varepsilon>0$.

Ces propriétés de convergence permettent de justifier que $\operatorname{div}\left(I_{2^{-k}} u_{k} \theta_{k}\right)$ tend vers $\operatorname{div}(u \theta)$ au sens des distributions pourvu que $1-\frac{N}{p}+\frac{N}{2}>0$, condition qui est équivalente à $p>2 N /(N+2)$. Le passage à la limite dans le terme 
$\operatorname{div}\left(I_{2^{-k}} u_{k} \otimes u_{k}\right)$ se fait exactement comme pour le système de Navier-Stokes incompressible « classique ».

\section{Espaces fonctionnels et outils d'analyse harmonique}

Commençons par rappeler la définition des espaces de type $L^{p}$ faible.

DÉfinition 3.1. - Pour $1 \leq p<\infty$, on définit l'espace $L^{p}$ faible (noté $L^{p, \infty}$ par la suite) comme l'ensemble des fonctions mesurables de $\mathbb{R}^{N}$ dans $\mathbb{R}$ telles que

$$
\|f\|_{L^{p, \infty}}:=\sup _{\lambda>0} \lambda\left|\left\{x \in \mathbb{R}^{N} /|f(x)|>\lambda\right\}\right|^{\frac{1}{p}}<\infty .
$$

Remarque 3.2. - Dans le cas $1<p<\infty$, l'espace $L^{p, \infty}$ coïncide avec l'espace de Lorentz défini par l'interpolation réelle $\left(L^{\infty}, L^{1}\right)_{\left(\frac{1}{p}, \infty\right)}$. En d'autres termes, $f$ est dans $L^{p, \infty}$ si et seulement si, pour tout $A>0$, on peut écrire $f=f^{A}+f_{A}$ avec $\left\|f_{A}\right\|_{L^{1}} \leq C A^{1-1 / p}$ et $\left\|f^{A}\right\|_{L^{\infty}} \leq C A^{-1 / p}$, et la « meilleure constante » $C$ est une norme équivalente à la quantité de la définition ci-dessus (voir par exemple [22]).

Plus généralement, pour $1 \leq q \leq \infty$, l'espace de Lorentz $L^{p, q}$ peut être défini par interpolation réelle en posant :

$$
L^{p, q}:=\left(L^{\infty}, L^{1}\right)_{\left(\frac{1}{p}, q\right)} .
$$

Dans la suite de cette partie, nous rappelons brièvement la définition de la décomposition de Littlewood-Paley (selon [6]) ainsi que celle des espaces de Besov.

La décomposition de Littlewood-Paley se définit à l'aide d'une décomposition dyadique de l'unité : soit $\chi$ une fonction positive radiale de classe $\mathcal{C}^{\infty}$, supportée dans la boule $\left\{|\xi| \leq \frac{4}{3}\right\}$, valant 1 sur $\left\{|\xi| \leq \frac{3}{4}\right\}$, et telle que $r \mapsto \chi\left(r e_{r}\right)$ soit décroissante. On pose $\varphi(\xi)=\chi(\xi / 2)-\chi(\xi)$ de telle sorte que

$$
\forall \xi \in \mathbb{R}^{N} \backslash\{0\}, \sum_{q \in \mathbb{Z}} \varphi\left(2^{-q} \xi\right)=1 \quad \text { et } \quad \forall \xi \in \mathbb{R}^{N}, \chi(\xi)=1-\sum_{q \in \mathbb{N}} \varphi\left(2^{-q} \xi\right) \text {. }
$$

On définit ensuite les opérateurs de localisation en fréquences $\dot{\Delta}_{q}$ et $\dot{S}_{q}$ de $\mathcal{L}\left(\mathcal{S}^{\prime} ; \mathcal{S}\right)$ par :

$$
\dot{\Delta}_{q} u:=\varphi\left(2^{-q} D\right) u \quad \text { et } \quad \dot{S}_{q} u:=\chi\left(2^{-q} D\right) u \text { pour tout } q \in \mathbb{Z} .
$$

Notons que pour une distribution tempérée $u \in \mathcal{S}^{\prime}\left(\mathbb{R}^{N}\right)$, les fonctions $\dot{\Delta}_{q} u$ et $\dot{S}_{q} u$ sont analytiques à croissance lente. Si de plus il existe un réel $s$ tel que $u \in H^{s}\left(\mathbb{R}^{N}\right)$ alors $\dot{\Delta}_{q} u$ et $\dot{S}_{q} u$ appartiennent à l'espace $H^{\infty}:=\bigcap_{\sigma \in \mathbb{R}} H^{\sigma}$. 
Par ailleurs, on établit à l'aide de (14) que $u=\dot{S}_{0} u+\sum_{q \in \mathbb{N}} \dot{\Delta}_{q} u$ dans $\mathcal{S}^{\prime}\left(\mathbb{R}^{N}\right)$ et $u=\sum_{q \in \mathbb{Z}} \dot{\Delta}_{q} u$ dans $\mathcal{S}^{\prime}\left(\mathbb{R}^{N}\right)$ modulo un polynôme (voir par exemple $[22])$.

Enfin, nous utiliserons souvent la propriété de presque orthogonalité suivante :

(15) $\quad \dot{\Delta}_{k} \dot{\Delta}_{q} u \equiv 0 \quad$ si $\quad|k-q| \geq 2 \quad$ et $\quad \dot{\Delta}_{k}\left(\dot{S}_{q-1} u \dot{\Delta}_{q} v\right) \equiv 0 \quad$ si $\quad|k-q| \geq 5$.

Nous pouvons maintenant définir les espaces de Besov homogènes comme suit :

DÉfinition 3.3. - Soient $s \in \mathbb{R},(p, r) \in[1, \infty]^{2}$ et $u \in \mathcal{S}^{\prime}\left(\mathbb{R}^{N}\right)$. On note $\|u\|_{\dot{B}_{p, r}^{s}} \stackrel{\text { déf }}{=}\left(\sum_{q \in \mathbb{Z}} 2^{r q s}\left\|\dot{\Delta}_{q} u\right\|_{L^{p}}^{r}\right)^{\frac{1}{r}} \quad$ si $\quad r<\infty, \quad$ et $\|u\|_{\dot{B}_{p, \infty}^{s}} \stackrel{\text { déf }}{=} \sup _{q \in \mathbb{Z}} 2^{q s}\left\|\dot{\Delta}_{q} u\right\|_{L^{p}}$.

On définit l'espace de Besov homogène $\dot{B}_{p, r}^{s}:=\dot{B}_{p, r}^{s}\left(\mathbb{R}^{N}\right)$ par

- $\dot{B}_{p, r}^{s}=\left\{u \in \mathcal{S}^{\prime}\left(\mathbb{R}^{N}\right) \mid\|u\|_{\dot{B}_{p, r}^{s}}<+\infty\right\}$, lorsque $s<\frac{N}{p}$ ou $s=\frac{N}{p}$ et $r=1$.

- $\dot{B}_{p, r}^{s}=\left\{u \in \mathcal{S}^{\prime}\left(\mathbb{R}^{N}\right)|\forall| \alpha \mid=k+1, \quad \partial^{\alpha} u \in \dot{B}_{p, r}^{s-k-1}\right\}$ si $\frac{N}{p}+k \leq s<$ $\frac{N}{p}+k+1$, ou $s=\frac{N}{p}+k+1$ et $r=1$, pour un $k \in \mathbb{N}$.

REMARQUE 3.4. - On prendra garde au fait que $\dot{B}_{p, r}^{s}\left(\mathbb{R}^{N}\right)$ est un espace de Banach si et seulement si $s<N / p$ ou $s \leq N / p$ et $r=1$.

Rappelons également la définition des espaces de Besov non homogènes :

DÉfinition 3.5. - Soient $s \in \mathbb{R},(p, r) \in[1, \infty]^{2}$ et $u \in \mathcal{S}^{\prime}\left(\mathbb{R}^{N}\right)$. On note

$$
\begin{aligned}
\|u\|_{B_{p, r}^{s}} & :=\left(\left\|\dot{S}_{0} u\right\|_{L^{p}}^{r}+\sum_{q \in \mathbb{N}} 2^{r q s}\left\|\dot{\Delta}_{q} u\right\|_{L^{p}}^{r}\right)^{\frac{1}{r}} \quad \text { si } r<\infty \\
\|u\|_{B_{p, \infty}^{s}} & :=\max \left(\left\|\dot{S}_{0} u\right\|_{L^{p}}, \sup _{q \in \mathbb{N}} 2^{q s}\left\|\dot{\Delta}_{q} u\right\|_{L^{p}}\right) .
\end{aligned}
$$

L'espace de Besov non homogène $B_{p, r}^{s}:=B_{p, r}^{s}\left(\mathbb{R}^{N}\right)$ est l'ensemble des distributions tempérées $u$ telles que $\|u\|_{B_{p, r}^{s}}$ soit fini.

Remarque 3.6. - Les espaces de Besov $B_{2,2}^{s}$ et $\dot{B}_{2,2}^{s}$ coïncident respectivement avec les espaces de Sobolev $H^{s}$ et $\dot{H}^{s}$. Si $s \in \mathbb{R}^{+} \backslash \mathbb{N}$, les espaces de Besov $B_{\infty, \infty}^{s}$ et $\dot{B}_{\infty, \infty}^{s}$ coïncident respectivement avec les espaces de Hölder $C^{s}$ et $\dot{C}^{s}$. Par abus, nous noterons $C^{s}:=B_{\infty, \infty}^{s}$ et $\dot{C}^{s}:=\dot{B}_{\infty, \infty}^{s}$ pour tout $s \in \mathbb{R}$.

Les inégalités suivantes, dites de Bernstein et démontrées par exemple dans [6] seront d'un usage constant.

Lemme 3.7. - Soit $1 \leq p_{1} \leq p_{2} \leq \infty$ et $\psi \in \mathcal{C}_{c}^{\infty}\left(\mathbb{R}^{N}\right)$. Alors on a

$$
\left\|\psi\left(2^{-q} D\right) u\right\|_{L^{p_{2}}} \leq C 2^{q N\left(\frac{1}{p_{1}}-\frac{1}{p_{2}}\right)}\left\|\psi\left(2^{-q} D\right) u\right\|_{L^{p_{1}}} .
$$


Comme conséquence de l'inégalité de Bernstein et de la définition de $\dot{B}_{p, r}^{s}$, on a la proposition suivante :

Proposition 3.8. - (i) Il existe une constante $c$ strictement positive telle que

$$
c^{-1}\|u\|_{\dot{B}_{p, r}^{s}} \leq\|\nabla u\|_{\dot{B}_{p, r}^{s-1}} \leq c\|u\|_{\dot{B}_{p, r}^{s}}
$$

(ii) Pour $1 \leq p_{1} \leq p_{2} \leq \infty$ et $1 \leq r_{1} \leq r_{2} \leq \infty$, on a $\dot{B}_{p_{1}, r_{1}}^{s} \hookrightarrow \dot{B}_{p_{2}, r_{2}}^{s-N\left(\frac{1}{p_{1}}-\frac{1}{p_{2}}\right)}$. (iii) Si $p \in[1, \infty]$, alors $\dot{B}_{p, 1}^{\frac{N}{p}} \hookrightarrow \dot{B}_{p, \infty}^{\frac{N}{p}} \cap L^{\infty}$. Si $p$ est fini, l'espace $\dot{B}_{p, 1}^{\frac{N}{p}}$ est une algèbre.

(iv) Interpolation réelle : $\left(\dot{B}_{p, r}^{s_{1}}, \dot{B}_{p, r}^{s_{2}}\right)_{\theta, r^{\prime}}=\dot{B}_{p, r^{\prime}}^{\theta s_{2}+(1-\theta) s_{1}}$ pour $0<\theta<1$ et $1 \leq p, r, r^{\prime} \leq \infty$.

Signalons aussi le résultat d'inclusion suivant qui nous sera fort utile :

Lemme 3.9. - Pour $1<p<q \leq \infty$, on a

$$
L^{p, \infty}\left(\mathbb{R}^{N}\right) \hookrightarrow \dot{B}_{q, \infty}^{\frac{N}{q}-\frac{N}{p}}\left(\mathbb{R}^{N}\right) .
$$

Démonstration. - Notons $h_{\jmath}=2^{{ }^{N}} h\left(2^{\jmath} \cdot\right)$ avec $h=\mathcal{F}^{-1} \varphi$. On a $\dot{\Delta}_{\jmath} u=h_{\jmath} \star u$, donc par les inégalités de convolution dans les espaces de Lorentz (voir par exemple [22]),

$$
\left\|\dot{\Delta}_{\jmath} u\right\|_{L^{q}} \leq\left\|h_{\jmath}\right\|_{L^{r, 1}}\|u\|_{L^{p, \infty}} \quad \text { avec } \quad \frac{1}{r}=1+\frac{1}{q}-\frac{1}{p} .
$$

En faisant un changement de variable, on constate que

$$
\left\|h_{\jmath}\right\|_{L^{r, 1}}=2^{\jmath N\left(1-\frac{1}{r}\right)}\|h\|_{L^{r, 1}}
$$

d'où le résultat.

Nous souhaitons maintenant donner quelques estimations a priori dans les espaces de Besov pour l'équation de la chaleur. Ces estimations découlent du lemme suivant démontré dans [8] :

Lemme 3.10. - Il existe deux constantes $c$ et $C$ telles que pour tout $\tau \geq 0$, $q \in \mathbb{Z}$, et $p \in[1, \infty]$, on ait

$$
\left\|e^{\tau \Delta} \dot{\Delta}_{q} u\right\|_{L^{p}} \leq C e^{-c \tau 2^{2 q}}\left\|\dot{\Delta}_{q} u\right\|_{L^{p}} .
$$

De ce lemme, on déduit facilement (voir encore [8]) le résultat suivant : 
Proposition 3.11. - Soit $s \in \mathbb{R}, 1 \leq p, r, \rho_{1} \leq \infty$. Soit $u_{0} \in \dot{B}_{p, r}^{s}$ et $f \in$ $\widetilde{L}_{T}^{\rho_{1}}\left(\dot{B}_{p, r}^{s-2+\frac{2}{\rho_{1}}}\right)$. Alors l'équation de la chaleur

$$
\partial_{t} u-\nu \Delta u=f, \quad u_{\mid t=0}=u_{0}
$$

admet une unique solution $u$ dans $\widetilde{L}_{T}^{\infty}\left(\dot{B}_{p, r}^{s}\right) \cap \widetilde{L}_{T}^{\rho_{1}}\left(\dot{B}_{p, r}^{s+\frac{2}{\rho_{1}}}\right)$ et il existe une constante $C$ ne dépendant que de la dimension $N$ telle que l'on ait l'estimation a priori suivante pour tout $t \in[0, T]$ et $\rho \geq \rho_{1}$ :

$$
\nu^{\frac{1}{\rho}}\|u\|_{\widetilde{L}_{t}^{\rho}\left(\dot{B}_{p, r}^{s+\frac{2}{\rho}}\right)} \leq C\left(\left\|u_{0}\right\|_{\dot{B}_{p, r}^{s}}+\nu^{\frac{1}{\rho_{1}}-1}\|f\|_{\widetilde{L}_{t}^{\rho_{1}}\left(\dot{B}_{p, r}^{\left.s-2+\frac{2}{\rho_{1}}\right)}\right)}\right) .
$$

Si $r<\infty$, la solution u appartient de plus à $\mathcal{C}\left([0, T] ; \dot{B}_{p, r}^{s}\right)$.

Dans la proposition ci-dessus, on a utilisé les espaces $\widetilde{L}_{T}^{\rho}\left(\dot{B}_{p, r}^{s}\right)$ qui se définissent comme à la définition 3.3 après avoir posé

$$
\|u\|_{\widetilde{L}_{T}^{\rho}\left(\dot{B}_{p, r}^{s}\right)}:=\left\|2^{q s}\right\| \dot{\Delta}_{q} u\left\|_{L_{T}^{\rho}\left(L^{p}\right)}\right\|_{\ell^{r}(\mathbb{Z})} .
$$

Notons qu'en vertu de l'inégalité de Minkowski, on a

$$
\|u\|_{L_{T}^{\rho}\left(\dot{B}_{p, r}^{s}\right)} \leq\|u\|_{\widetilde{L}_{T}^{\rho}\left(\dot{B}_{p, r}^{s}\right)} \quad \text { pour } \quad \rho \geq r
$$

et l'inégalité opposée si $\rho \leq r$.

On posera $\widetilde{\mathcal{C}}_{T}\left(\dot{B}_{p, r}^{s}\right):=\widetilde{L}_{T}^{\infty}\left(\dot{B}_{p, r}^{s}\right) \cap \mathcal{C}\left([0, T] ; \dot{B}_{p, r}^{s}\right)$ et $\widetilde{L}_{l o c}^{\rho}\left(\mathbb{R}_{+} ; \dot{B}_{p, r}^{s}\right)=$ $\bigcap_{T>0} \widetilde{L}_{T}^{\rho}\left(\dot{B}_{p, r}^{s}\right)$.

Sur le même modèle, on peut définir des espaces non homogènes $\widetilde{L}_{T}^{\rho}\left(B_{p, r}^{s}\right)$. Dans le cas particulier où $p=r=2$, on utilisera plutôt la notation $\widetilde{L}_{T}^{\rho}\left(\dot{H}^{s}\right)$ ou $\widetilde{L}_{T}^{\rho}\left(H^{s}\right)$.

Remarque 3.12. - Grâce à la proposition 3.11, et en utilisant le fait que le projecteur $\mathcal{P}$ sur les champs à divergence nulle est un multiplicateur de Fourier homogène de degré 0 et est donc continu de $\dot{B}_{p, r}^{s}$ dans lui-même (voir e.g. [22]), il est facile de résoudre le système de Stokes non stationnaire

$$
\left\{\begin{array}{l}
\partial_{t} u-\nu \Delta u+\nabla \Pi=f, \quad \operatorname{div} u=0, \\
u_{\mid t=0}=u_{0},
\end{array}\right.
$$

avec donnée initiale $u_{0} \in \dot{B}_{p, r}^{s}$ à divergence nulle, et force $f \in \widetilde{L}_{T}^{1}\left(\dot{B}_{p, r}^{s}\right)$.

On obtient encore une unique solution $(u, \nabla \Pi)$ avec $u \in \widetilde{L}_{T}^{\infty}\left(\dot{B}_{p, r}^{s}\right) \cap$ $\widetilde{L}_{T}^{1}\left(\dot{B}_{p, r}^{s+2}\right)$ et $\nabla \Pi \in \widetilde{L}_{T}^{1}\left(\dot{B}_{p, r}^{s}\right)$, et $u$ vérifie pour tout $1 \leq \rho \leq \infty$ l'estimation

$$
\nu^{\frac{1}{\rho}}\|u\|_{\widetilde{L}_{T}^{\rho}\left(\dot{B}_{p, r}^{s+\frac{2}{\rho}}\right)} \leq C\left(\left\|u_{0}\right\|_{\dot{B}_{p, r}^{s}}+\|\mathcal{P} f\|_{\widetilde{L}_{T}^{1}\left(\dot{B}_{p, r}^{s}\right)}\right) .
$$

Si $r<\infty$, la solution $u$ appartient de plus à $\mathcal{C}\left([0, T] ; \dot{B}_{p, r}^{s}\right)$. 
REmarque 3.13. - On peut énoncer une version non homogène de la proposition 3.11 pour des données $u_{0} \in B_{p, r}^{s}$ et $f \in \widetilde{L}_{T}^{\rho_{1}}\left(B_{p, r}^{s-2+\frac{2}{\rho_{1}}}\right)$. Les résultats d'existence, d'unicité et de continuité en temps demeurent, mais la constante $C$ de (17) se met à dépendre (au pire linéairement) de $T$.

La preuve de certaines estimations a priori pour des termes de type quadratique sera grandement facilitée par l'usage du calcul paradifférentiel et notamment de la décomposition suivante introduite par J.-M. Bony dans [3] :

$$
f g=\dot{T}_{f} g+\dot{T}_{g} f+\dot{R}(f, g),
$$

où le terme de paraproduit $\dot{T}$ est défini par $\dot{T}_{f} g:=\sum_{q} \dot{S}_{q-1} f \dot{\Delta}_{q} g$, et le terme de reste $\dot{R}$, par $\dot{R}(f, g):=\sum_{q} \dot{\Delta}_{q} f \widetilde{\Delta}_{q} g$ avec $\widetilde{\Delta}_{q}:=\dot{\Delta}_{q-1}+\dot{\Delta}_{q}+\dot{\Delta}_{q+1}$.

Nous énonçons ci-dessous quelques résultats de continuité d'usage constant pour les opérateurs de paraproduit et de reste (consulter par exemple [28] pour une étude exhaustive de ces opérateurs).

Proposition 3.14. - Soit $1 \leq p, p_{1}, p_{2}, r, r_{1}, r_{2} \leq \infty$ vérifiant $\frac{1}{p}=\frac{1}{p_{1}}+\frac{1}{p_{2}}$ et $\frac{1}{r}=\frac{1}{r_{1}}+\frac{1}{r_{2}}$. L'opérateur de paraproduit $\dot{T}$ est continu :

- de $L^{\infty} \times \dot{B}_{p, r}^{t}$ dans $\dot{B}_{p, r}^{t}$ pour tout $t \in \mathbb{R}$,

- de $\dot{B}_{p_{1}, r_{1}}^{-s} \times \dot{B}_{p_{2}, r_{2}}^{t}$ dans $\dot{B}_{p, r}^{t-s}$ pour tout $t \in \mathbb{R}$ et $s>0$.

$L$ 'opérateur de reste $\dot{R}$ est continu :

- de $\dot{B}_{p_{1}, r_{1}}^{s} \times \dot{B}_{p_{2}, r_{2}}^{t}$ dans $\dot{B}_{p, r}^{s+t}$ pour tout $(s, t) \in \mathbb{R}^{2}$ tel que $s+t>0$,

- de $\dot{B}_{p_{1}, r_{1}}^{s} \times \dot{B}_{p_{2}, r_{2}}^{-s}$ dans $\dot{B}_{p, \infty}^{0}$ si $s \in \mathbb{R}$ et $\frac{1}{r_{1}}+\frac{1}{r_{2}} \geq 1$.

La proposition ci-dessus permet de montrer la plupart des résultats de continuité pour le produit de deux distributions appartenant à des espaces de Besov. Nous utiliserons fréquemment par exemple que l'application $(u, v) \mapsto u v$ est continue de

- $\dot{B}_{N, 1}^{s} \times \dot{B}_{N, 1}^{t}$ dans $\dot{B}_{N, 1}^{s+t-1}$ si $-1<s, t \leq 1$ et $s+t>0$,

$-\dot{H}^{s} \times \dot{H}^{t}$ dans $\dot{B}_{2,1}^{s+t-\frac{N}{2}}$ si $|s|<\frac{N}{2},|t|<\frac{N}{2}$ et $s+t>0$.

\section{Quelques estimations a priori}

4.1. Espaces de Lorentz et système de Stokes non stationnaire. - Dans cette section, on démontre quelques estimations a priori dans les espaces de Lorentz pour le système de Stokes non stationnaire (20). Le lemme suivant nous permettra de contrôler le terme de couplage avec l'équation de transport dans le système de Boussinesq.

Lemme 4.1. - On a les estimations a priori suivantes pour tout $t \geq 0$ :

TOME $136-2008-\mathrm{N}^{\mathrm{O}} 2$ 


$$
\begin{aligned}
& \text { - Si } N=3 \text { alors } \nu\left\|\int_{0}^{t} e^{\nu(t-s) \Delta} \mathcal{P}\left(\theta e_{3}\right)(s) d s\right\|_{L^{3, \infty}} \leq C\|\theta\|_{L_{t}^{\infty}\left(L^{1}\right)} . \\
& \text { - Si } N \geq 4 \text { alors } \nu\left\|\int_{0}^{t} e^{\nu(t-s) \Delta} \mathcal{P}\left(\theta e_{N}\right)(s) d s\right\|_{L^{N, \infty}} \leq C\|\theta\|_{L_{t}^{\infty}\left(L^{\frac{N}{3}, \infty}\right)}
\end{aligned}
$$

Démonstration. - Par un changement de variable temporel, on peut se ramener au cas $\nu=1$. Notons par ailleurs que $\mathcal{P} \in \mathcal{L}\left(L^{p}\left(\mathbb{R}^{N}\right) ; L^{p}\left(\mathbb{R}^{N}\right)\right)$ pour tout $1<p<\infty$. Par interpolation réelle, on a donc en particulier

$$
\mathcal{P} \in \mathcal{L}\left(L^{N, \infty}\left(\mathbb{R}^{N}\right), L^{N, \infty}\left(\mathbb{R}^{N}\right)\right) .
$$

Par conséquent, on peut « oublier » le projecteur $\mathcal{P}$ dans les inégalités à démontrer et se limiter à la preuve d'inégalités similaires pour l'opérateur de la chaleur. Pour ce faire, on écrit la décomposition suivante avec $A$ paramètre positif :

$$
\int_{0}^{t} e^{(t-s) \Delta} \theta(s) d s=\underbrace{\int_{0}^{A} 1_{[0, t]}(s) e^{(t-s) \Delta} \theta(s) d s}_{I_{A}}+\underbrace{\int_{A}^{\infty} 1_{[0, t]}(s) e^{(t-s) \Delta} \theta(s) d s}_{I^{A}} .
$$

Par calcul direct on a pour $\tau>0$ et $1 \leq p \leq q \leq \infty$,

$$
(22) \mid e^{\tau \Delta}\left\|_{\mathcal{L}\left(L^{p}, L^{q}\right)}=\right\| \mathcal{F}^{-1}\left(e^{-\tau|\xi|^{2}}\right)\left\|_{L^{r}}=\frac{1}{\tau^{N / 2}}\right\| G\left(\frac{x}{\sqrt{\tau}}\right)\left\|_{L^{r}}=\frac{1}{\tau^{N / 2 r^{\prime}}}\right\| G \|_{L^{r}}
$$

où l'on a noté $G=\mathcal{F}^{-1}\left(e^{-|\xi|^{2}}\right), 1 / r=1+1 / q-1 / p$ et $1 / r^{\prime}=1 / p-1 / q$.

Supposons d'abord que $N=3$. On a

$$
\begin{aligned}
\left\|I_{A}\right\|_{L^{1}} & \leq \int_{0}^{A}\left\|e^{(t-s) \Delta} \theta(s)\right\|_{L^{1} 1_{[0, t]}}(s) d s, \\
& \leq \int_{0}^{A}\left\|e^{(t-s) \Delta}\right\|_{\mathcal{L}\left(L^{1}, L^{1}\right)}\|\theta(s)\|_{L^{1}} 1_{[0, t]}(s) d s, \\
& \leq\|G\|_{L^{1}} \int_{0}^{A}\|\theta(s)\|_{L^{1}} 1_{[0, t]}(s) d s \leq A\|G\|_{L^{1}}\|\theta\|_{L_{t}^{\infty}\left(L^{1}\right)} .
\end{aligned}
$$

D'autre part, pour $I^{A}$, on peut écrire

$$
\begin{aligned}
\left\|I^{A}\right\|_{L^{\infty}} & \leq \int_{A}^{\infty}\left\|e^{(t-s) \Delta} \theta(s)\right\|_{L^{\infty}} 1_{[0, t]}(s) d s \\
& \leq \int_{A}^{\infty}\left\|e^{(t-s) \Delta}\right\|_{\mathcal{L}_{\left(L^{1}, L^{\infty}\right)}}\|\theta(s)\|_{L^{1}} 1_{[0, t]}(s) d s \\
& \leq\|G\|_{L^{\infty}} \int_{A}^{\infty} \frac{1}{(t-s)^{3 / 2}}\|\theta(s)\|_{L^{1}} 1_{[0, t]}(s) d s \\
& \leq 2 A^{-1 / 2}\|G\|_{L^{\infty}}\|\theta\|_{L_{t}^{\infty}\left(L^{1}\right)} .
\end{aligned}
$$

En tenant compte de ces deux estimations, de la remarque 3.2 et de (21), on obtient le résultat souhaité dans le cas $N=3$. 
Le cas $N \geq 4$ se traite de manière analogue. À partir de (22), on montre par interpolation que l'opérateur $e^{\tau \Delta}$ est continu de $L^{\frac{N}{3}, \infty}$ dans $L^{\frac{N}{3}, \infty}$ avec norme bornée par $C\|G\|_{L^{1}}$. Cela assure que pour tout $A \geq 0$, on a

$$
\begin{aligned}
\left\|I_{A}\right\|_{L^{\frac{N}{3}, \infty}} & \leq \int_{0}^{A}\left\|e^{(t-s) \Delta}\right\|_{\mathcal{L}\left(L^{\frac{N}{3}, \infty}, L^{\frac{N}{3}, \infty}\right)}\|\theta(s)\|_{L^{\frac{N}{3}, \infty}} 1_{[0, t]}(s) d s, \\
& \leq C A\|G\|_{L^{1}\|\theta\|_{L_{t}^{\infty}\left(L^{\frac{N}{3}, \infty}\right)} .}
\end{aligned}
$$

Pour majorer $I^{A}$, on utilise le fait que l'opérateur $e^{\tau \Delta}$ est continu de $L^{\frac{N}{3}, \infty}$ dans $L^{\infty}$ avec norme $C \tau^{-\frac{3}{2}}\|G\|_{L^{\frac{N}{N-3}, 1}}$ (combiner (22) avec un argument d'interpolation). On peut donc écrire

$$
\begin{aligned}
\left\|I^{A}\right\|_{L^{\infty}} & \leq \int_{A}^{\infty}\left\|e^{(t-s) \Delta}\right\|_{\mathcal{L}\left(L^{\frac{N}{3}, \infty}, L^{\infty}\right)}\|\theta(s)\|_{L^{\frac{N}{3}, \infty}} 1_{[0, t]}(s) d s \\
& \leq C\|G\|_{L^{\frac{N}{N-3}, 1}} \int_{A}^{\infty} \frac{1}{(t-s)^{3 / 2}}\|\theta(s)\|_{L^{\frac{N}{3}, \infty}} 1_{[0, t]}(s) d s, \\
& \leq C A^{-1 / 2}\|G\|_{L^{\frac{N}{N-3}, 1}\|\theta\|_{L_{t}^{\infty}\left(L^{\frac{N}{3}, \infty}\right)} .}
\end{aligned}
$$

En combinant les majorations pour $I_{A}$ et $I^{A}$, le fait que

$$
\left(L^{\frac{N}{3}, \infty}, L^{\infty}\right)_{\frac{2}{3}, \infty}=L^{N, \infty},
$$

et (21), on peut maintenant conclure à l'inégalité souhaitée dans le cas $N \geq 4$.

Le lemme suivant (qui semble être dû à Y. Meyer, voir [26]) nous permettra de contrôler le terme de convection dans l'équation sur $u$.

Lemme 4.2. - Pour tout $t \geq 0$ et $\nu \geq 0$, on a les estimations a priori

$$
\nu\left\|\int_{0}^{t} e^{\nu(t-s) \Delta} \mathcal{P} \nabla g(s) d s\right\|_{L^{N, \infty}} \leq \begin{cases}C\|g\|_{L_{t}^{\infty}\left(L^{1}\right)} & \text { si } N=2, \\ C\|g\|_{L_{t}^{\infty}\left(L^{\frac{N}{2}, \infty}\right)} & \text { si } N \geq 3 .\end{cases}
$$

Démonstration. - Pour la commodité du lecteur, nous donnons ci-dessous les grandes lignes de la démonstration. On procède comme dans le lemme précédent : après s'être ramené au cas $\nu=1$, on découpe l'intégrale à majorer en deux parties $J_{A}$ et $J^{A}$. En vertu de (21), on peut «oublier» le projecteur $\mathcal{P}$. De plus, il est clair que pour tout $\tau>0$ et $1 \leq p \leq q \leq \infty$, on a

$$
\left\|e^{\tau \Delta} \nabla\right\|_{\mathcal{L}\left(L^{p}, L^{q}\right)}=\frac{1}{\tau^{\frac{1}{2}+\frac{N}{2 r^{\prime}}}}\|H\|_{L^{r}} \quad \text { avec } \quad H:=\mathcal{F}^{-1}\left(e^{-|\eta|^{2}} \eta\right) \quad \text { et } \quad \frac{1}{r^{\prime}}=\frac{1}{p}-\frac{1}{q} \text {. }
$$

En reprenant les calculs du lemme précédent, on obtient donc si $N \geq 3$,

$$
\left\|J_{A}\right\|_{L^{\frac{N}{2}, \infty}} \leq C A\|H\|_{L^{1}}\|g\|_{L^{\frac{N}{2}, \infty}} \text { et }\left\|J^{A}\right\|_{L^{\infty}} \leq C A^{-1}\|H\|_{L^{\frac{N}{N-2}, 1}}\|g\|_{L^{\frac{N}{2}, \infty}} \text {. }
$$

On conclut alors en remarquant que $\left(L^{\frac{N}{2}, \infty}, L^{\infty}\right)_{\frac{1}{2}, \infty}=L^{N, \infty}$. 
Le cas $N=2$ est formellement identique. Il suffit de remplacer $L^{\frac{N}{2}, \infty}$ par $L^{1}$.

4.2. Estimations a priori pour le système de Navier-Stokes. - Dans cette partie, on démontre des estimations a priori pour le système de Navier-Stokes incompressible :

$$
\left\{\begin{array}{l}
\partial_{t} u+u \cdot \nabla u-\nu \Delta u+\nabla \Pi=f \\
\operatorname{div} u=0 \\
u(0, x)=u_{0}(x)
\end{array}\right.
$$

sous diverses conditions de petitesse.

Énonçons tout d'abord un résultat global de propagation de la régularité $L_{t}^{\infty}\left(\dot{B}_{p, 1}^{-1+\frac{N}{p}}\right) \cap L_{t}^{1}\left(\dot{B}_{p, 1}^{1+\frac{N}{p}}\right)$ sous l'hypothèse que $u$ est petite dans $\dot{C}^{-1}$.

LEMmE 4.3. - Soit $p \in[1, \infty[$ et $u$ une solution de (24) appartenant $\grave{a}$ $L_{T}^{\infty}\left(\dot{B}_{p, 1}^{-1+\frac{N}{p}}\right) \cap L_{T}^{1}\left(\dot{B}_{p, 1}^{1+\frac{N}{p}}\right)$. Il existe deux constantes $c$ et $C$ telles que si $u_{0}$ appartient $\grave{a} \dot{B}_{p, 1}^{-1+\frac{N}{p}}$ et vérifie div $u_{0}=0$, si $f$ est dans $L^{1}\left([0, T] ; \dot{B}_{p, 1}^{-1+\frac{N}{p}}\right)$ et de plus

$$
\sup _{t \in[0, T]}\|u(t)\|_{\dot{C}^{-1}} \leq c \nu
$$

alors, on a pour tout $t \in[0, T]$ l'estimation

$$
\|u(t)\|_{\dot{B}_{p, 1}^{\frac{N}{p}-1}}+\nu \int_{0}^{t}\|u(\tau)\|_{\dot{B}_{p, 1}^{\frac{N}{p}+1}} d \tau \leq C\left(\left\|u_{0}\right\|_{\dot{B}_{p, 1}^{\frac{N}{p}-1}}+\int_{0}^{t}\|f(\tau)\|_{\dot{B}_{p, 1}^{\frac{N}{p}-1}} d \tau\right) .
$$

Démonstration. - On applique l'opérateur $\mathcal{P} \dot{\Delta}_{q}$ à l'équation de Navier-Stokes. Sachant que $\operatorname{div} u=0$, on obtient

$$
\partial_{t} \dot{\Delta}_{q} u-\nu \Delta \dot{\Delta}_{q} u=\mathcal{P} \dot{\Delta}_{q} f-\mathcal{P} \dot{\Delta}_{q}(u \cdot \nabla u) .
$$

Admettons un instant que

$$
\left\|\mathcal{P} \dot{\Delta}_{q}(u \cdot \nabla u)\right\|_{L^{p}} \lesssim a_{q} 2^{-q\left(-1+\frac{N}{p}\right)}\|u\|_{\dot{C}^{-1}}\|u\|_{\dot{B}_{p, 1}^{1+\frac{N}{p}}} \quad \text { avec } \quad \sum_{q \in \mathbb{Z}} a_{q}=1 .
$$

Alors en utilisant le lemme 3.10 , on a

$$
\begin{aligned}
2^{q\left(-1+\frac{N}{p}\right)}\left\|\dot{\Delta}_{q} u(t)\right\|_{L^{p}} \leq 2^{q\left(-1+\frac{N}{p}\right)} e^{-c \nu t 2^{2 q}}\left\|\dot{\Delta}_{q} u_{0}\right\|_{L^{p}} & \\
& +\int_{0}^{t} e^{-c \nu(t-\tau) 2^{2 q}}\left(2^{q\left(-1+\frac{N}{p}\right)}\left\|\dot{\Delta}_{q} f\right\|_{L^{p}}+a_{q}\|u\|_{\dot{C}^{-1}}\|u\|_{\dot{B}_{p, 1}^{-1+\frac{N}{p}}}\right) d \tau .
\end{aligned}
$$


Après sommation sur $q$, on trouve donc

$$
\begin{aligned}
\|u\|_{L_{t}^{\infty}\left(\dot{B}_{p, 1}^{\frac{N}{p}-1}\right)}+\nu\|u\|_{L_{t}^{1}\left(\dot{B}_{p, 1}^{\frac{N}{p}+1}\right)} \leq & C\left(\left\|u_{0}\right\|_{\dot{B}_{p, 1}^{-1+\frac{N}{p}}}\right. \\
& \left.+\|f\|_{L_{t}^{1}\left(\dot{B}_{p, 1}^{-1+\frac{N}{p}}\right)}+\|u\|_{L_{t}^{\infty}\left(\dot{C}^{-1}\right)}\|u\|_{L_{t}^{1}\left(\dot{B}_{p, 1}^{1+\frac{N}{p}}\right)}\right) .
\end{aligned}
$$

Si l'on suppose que

$$
C\|u\|_{L_{t}^{\infty}\left(\dot{C}^{-1}\right)} \leq \frac{\nu}{2}
$$

alors on obtient l'estimation souhaitée.

L'inégalité (25) que nous avons utilisée plus haut repose sur le lemme suivant :

Lemme 4.4. - Soient $s>1, r \in[1,+\infty]$ et $u$ un champ à divergence nulle. Alors il existe une constante $C$ et une suite $a_{q} \in \ell^{r}(\mathbb{Z})$ telles que

$\left\|\dot{\Delta}_{q}(u \cdot \nabla v)\right\|_{L^{p}} \leq C 2^{-q(s-2)} a_{q}\left(\|u\|_{\dot{C}^{-1}}\|v\|_{\dot{B}_{p, r}^{s}}+\|v\|_{\dot{C}^{-1}}\|u\|_{\dot{B}_{p, r}^{s}}\right) \quad$ et $\quad\left\|\left(a_{q}\right)\right\|_{\ell^{r}} \leq 1$.

Démonstration. - En vertu de (15) et de $\operatorname{div} u=0$, on a

$$
\dot{\Delta}_{q}(u \cdot \nabla v)=\sum_{q^{\prime} \geq q-4} \dot{\Delta}_{q} \operatorname{div}\left(\dot{S}_{q^{\prime}+2} u \otimes \dot{\Delta}_{q^{\prime}} v\right)+\sum_{\left|q-q^{\prime}\right| \leq 4} \dot{\Delta}_{q}\left(\dot{\Delta}_{q^{\prime}} u \cdot \nabla \dot{S}_{q^{\prime}-1} v\right) .
$$

Sachant que $s>1$, on a

$$
\begin{aligned}
\sum_{q^{\prime} \geq q-4}\left\|\dot{\Delta}_{q} \operatorname{div}\left(\dot{S}_{q^{\prime}+2} u \otimes \dot{\Delta}_{q^{\prime}} v\right)\right\|_{L^{p}} & \leq C 2^{q} \sum_{q^{\prime} \geq q-4}\left\|\dot{S}_{q^{\prime}+2} u\right\|_{L^{\infty}}\left\|\dot{\Delta}_{q^{\prime}} v\right\|_{L^{p}} \\
& \leq C 2^{q} \sum_{q^{\prime} \geq q-4} 2^{-q^{\prime}(s-1)}\|u\|_{\dot{C}^{-1}} 2^{q^{\prime} s}\left\|\dot{\Delta}_{q^{\prime}} v\right\|_{L^{p}} \\
& \leq C 2^{-q(s-2)} a_{q}\|u\|_{\dot{C}^{-1}}\|v\|_{\dot{B}_{p, r}^{s}}
\end{aligned}
$$

avec $a_{q} \in \ell^{r}(\mathbb{Z})$ vérifiant $\left\|\left(a_{q}\right)\right\|_{\ell^{r}} \leq 1$,

D'autre part, on dispose de la majoration suivante :

$$
\begin{aligned}
\sum_{\left|q^{\prime}-q\right| \leq 4}\left\|\dot{\Delta}_{q}\left(\dot{\Delta}_{q^{\prime}} u \cdot \nabla \dot{S}_{q^{\prime}-1} v\right)\right\|_{L^{p}} & \leq C \sum_{\left|q^{\prime}-q\right| \leq 4}\left\|\dot{\Delta}_{q^{\prime}} u\right\|_{L^{p}}\left\|\dot{S}_{q^{\prime}-1} \nabla v\right\|_{L^{\infty}} \\
& \leq C 2^{-q(s-2)} a_{q}\|v\|_{\dot{C}^{-1}}\|u\|_{\dot{B}_{p, r}^{s}}
\end{aligned}
$$

d'où le lemme.

Il n'est pas clair que le lemme 4.3 demeure valable dans le cas limite $p=+\infty$. Mais on dispose toutefois du substitut suivant : 
LEMME 4.5. - Soit $u$ une solution de (24) avec donnée initiale $u_{0} \in \dot{B}_{\infty, 1}^{-1}$ $\grave{a}$ divergence nulle, et terme de force $f \in L^{1}\left([0, T], \dot{B}_{\infty, 1}^{-1}\right)$. Il existe deux constantes $c$ et $C$ telles que si

$$
\sup _{t \in[0, T]}\|u(t)\|_{L^{N, \infty}} \leq c \nu
$$

alors on a pour tout $t \in[0, T]$ l'estimation

$$
\|u(t)\|_{\dot{B}_{\infty, 1}^{-1}}+\nu\|u\|_{L_{t}^{1}\left(\dot{B}_{\infty, 1}^{1}\right)} \leq C\left(\left\|u_{0}\right\|_{\dot{B}_{\infty, 1}^{-1}}+\|f\|_{L_{t}^{1}\left(\dot{B}_{\infty, 1}^{-1}\right)}\right) .
$$

Démonstration. - La démarche générale est la même que dans la preuve du lemme 4.3 , mais on ne peut plus utiliser le lemme 4.4 qui est faux dans le cas limite $p=\infty, r=1$ et $s=1$. Le bon substitut est l'inégalité suivante :

$$
\left\|\dot{\Delta}_{q}(u \cdot \nabla u)\right\|_{L^{\infty}} \leq C a_{q} 2^{q}\|u\|_{L^{N, \infty}}\|u\|_{\dot{B}_{\infty, 1}^{1}} \quad \text { avec } \quad \sum_{q \in \mathbb{Z}} a_{q} \leq 1 .
$$

La justification de (26) repose sur la décomposition de Bony. En tenant compte de $\operatorname{div} u=0$, on a pour tout $j \in\{1, \cdots, N\}$,

$$
[\operatorname{div}(u \otimes u)]^{j}=\partial_{i}\left(\dot{T}_{u^{i}} u^{j}+\dot{T}_{u^{j}} u^{i}+\dot{R}\left(u^{i}, u^{j}\right)\right) .
$$

Les résultats de continuité pour le paraproduit énoncés dans la proposition 3.14 assurent que

$$
\left\|\operatorname{div} \dot{\Delta}_{q}\left(\dot{T}_{u} u\right)\right\|_{L^{\infty}} \leq C 2^{q} a_{q}\|u\|_{\dot{C}^{-1}}\|u\|_{\dot{B}_{\infty, 1}^{1}} \quad \text { avec } \quad \sum_{q \in \mathbb{Z}} a_{q} \leq 1
$$

et l'on a vu que $L^{N, \infty} \hookrightarrow \dot{C}^{-1}$ (cf lemme 3.9).

La continuité de l'injection de $L^{N, \infty}$ dans $\dot{C}^{-1}$ permet aussi d'écrire que $\left\|\dot{\Delta}_{q} \operatorname{div} \dot{R}(u, u)\right\|_{L^{\infty}} \leq C 2^{q}\left\|\dot{\Delta}_{q} \operatorname{div} \dot{R}(u, u)\right\|_{L^{N, \infty}} \quad$ pour tout $\quad q \in \mathbb{Z}$.

Par conséquent, on a

$$
\begin{aligned}
\left\|\dot{\Delta}_{q} \operatorname{div} \dot{R}(u, u)\right\|_{L^{\infty}} & \leq C 2^{2 q}\left\|\sum_{q^{\prime} \geq q-3} \dot{\Delta}_{q}\left(\dot{\Delta}_{q^{\prime}} u \widetilde{\Delta}_{q^{\prime}} u\right)\right\|_{L^{N, \infty}} \\
& \leq C 2^{2 q} \sum_{q^{\prime} \geq q-3}\left\|\dot{\Delta}_{q^{\prime}} u\right\|_{L^{N, \infty}}\left\|\widetilde{\Delta}_{q^{\prime}} u\right\|_{L^{\infty}} \\
& \leq C 2^{q} \sum_{q^{\prime} \geq q-3} 2^{q-q^{\prime}} a_{q^{\prime}}\|u\|_{L^{N, \infty}}\|u\|_{\dot{B}_{\infty, 1}^{1}}
\end{aligned}
$$

avec $\sum_{q} a_{q} \leq 1$, ce qui achève la démonstration de (26). 
4.3. Estimations pour l'équation de transport. - Dans cette section nous établissons diverses estimations a priori pour l'équation de transport

$$
\left\{\begin{array}{l}
\partial_{t} \theta+\operatorname{div}(\theta u)=f \\
\theta(0, x)=\theta_{0}(x)
\end{array}\right.
$$

avec $u$ champ de vecteur lipschitizien à divergence nulle. Pour commencer, rappelons un résultat classique relatif aux espaces $L^{p}$ ou $L^{p, \infty}$ :

Proposition 4.6. - Pour tout $p \in[1, \infty]$, on a les estimations a priori suivantes :

$$
\forall t \in \mathbb{R}^{+},\|\theta(t)\|_{L^{p}} \leq\left\|\theta_{0}\right\|_{L^{p}}+\int_{0}^{t}\|f(s)\|_{L^{p}} d s .
$$

Plus généralement, pour $1 \leq p<\infty$, on a

$$
\forall t \in \mathbb{R}^{+},\|\theta(t)\|_{L^{p, \infty}} \leq\left\|\theta_{0}\right\|_{L^{p, \infty}}+\int_{0}^{t}\|f(s)\|_{L^{p, \infty}} d s .
$$

Dans tous les cas, l'inégalité peut être remplacée par une égalité si $f \equiv 0$.

Démonstration. - En introduisant le flot $\psi$ du champ $u$ défini par

$$
\left\{\begin{array}{l}
\partial_{t} \psi(t, x)=u(t, \psi(t, x)), \\
\psi(0, x)=x
\end{array}\right.
$$

on obtient

$$
\theta(t, x)=\theta_{0}\left(\psi(t, x)^{-1}\right)+\int_{0}^{t} f\left(s, \psi\left(s, \psi(t, x)^{-1}\right)\right) d s .
$$

Sachant que $\operatorname{div} u=0$, la fonction $\psi_{t}:=\psi(t, \cdot)$ est un difféomorphisme qui préserve le volume. En conséquence,

$$
\|\theta(t)\|_{L^{p}} \leq\left\|\theta_{0}\right\|_{L^{p}}+\int_{0}^{t}\|f(s)\|_{L^{p}}
$$

avec égalité si $f \equiv 0$.

La preuve de l'inégalité dans les espaces $L^{p, \infty}$ se traite en comparant les ensembles de niveau de $\theta$ et de $\theta_{0}$. En effet : dans le cas $f \equiv 0$, on a pour tout $\lambda \geq 0$

$$
\left\{y \in \mathbb{R}^{N} /|\theta(t, y)|>\lambda\right\}=\psi_{t}\left(\left\{x \in \mathbb{R}^{N} /\left|\theta_{0}(x)\right|>\lambda\right\}\right),
$$

et les deux ensembles ont même mesure puisque $\psi$ préserve la mesure.

Le cas général $f \not \equiv 0$ s'en déduit facilement à partir de la formule (29).

Dans la proposition ci-dessous, nous énonçons un résultat de propagation de régularité de type Besov pour les équations de transport.

TOME $136-2008-\mathrm{N}^{\mathrm{O}} 2$ 
Proposition 4.7. - Soient $1 \leq p \leq p_{1} \leq+\infty, r \in[1, \infty]$ et $s \in \mathbb{R}$ tels que

$$
-1-\min \left(\frac{N}{p_{1}}, \frac{N}{p^{\prime}}\right)<s<1+\frac{N}{p_{1}} \text {. }
$$

Supposons que le champ de vecteurs $u$ soit à divergence nulle et à coefficients dans $L^{1}\left([0, T] ; \dot{B}_{p_{1}, 1}^{1+\frac{N}{p_{1}}}\left(\mathbb{R}^{N}\right)\right)$, que le terme de force $f$ appartienne $\grave{a} L^{1}\left([0, T] ; \dot{B}_{p, r}^{s}\left(\mathbb{R}^{N}\right)\right)$ et que la donnée initiale $\theta_{0}$ soit dans $\dot{B}_{p, r}^{s}\left(\mathbb{R}^{N}\right)$. Alors l'équation (27) admet une unique solution $\theta \in L^{\infty}\left([0, T] ; \dot{B}_{p, r}^{s}\right)$ $\left(\theta \in \mathcal{C}\left([0, T] ; \dot{B}_{p, r}^{s}\right)\right.$ si $\left.r<\infty\right)$ qui vérifie l'estimation

$$
\|\theta\|_{\widetilde{L}_{t}^{\infty}\left(\dot{B}_{p, r}^{s}\right)} \leq\left(\left\|\theta_{0}\right\|_{\dot{B}_{p, r}^{s}}+\|f\|_{\widetilde{L}_{t}^{1}\left(\dot{B}_{p, r}^{s}\right)}\right) \exp \left(C \int_{0}^{t}\|\nabla u\|_{\dot{B}_{p_{1}, 1}^{N}} d \tau\right) .
$$

L'inégalité ci-dessus reste valable dans le cas limite $r=\infty$ et $s=-1-$ $\min \left(\frac{N}{p_{1}}, \frac{N}{p^{\prime}}\right)$.

Démonstration. - Un résultat analogue a été établi dans [13] (mais dans le cadre des espaces de Besov non homogènes et sans le cas limite). Nous nous limitons donc à une preuve succincte des estimations a priori.

Appliquons $\dot{\Delta}_{q}$ à l'équation de transport (27). En utilisant la décomposition de Bony, la propriété de divergence nulle et (15), on obtient

$$
\partial_{t} \dot{\Delta}_{q} \theta+\dot{S}_{q-1} u \cdot \nabla \dot{\Delta}_{q} \theta=\dot{\Delta}_{q} f-F_{q}
$$

avec $F_{q}=\dot{\Delta}_{q}\left(\dot{T}_{\partial_{i} \theta} u^{i}\right)+\partial_{i} \dot{\Delta}_{q} \dot{R}\left(u^{i}, \theta\right)$

$$
+\sum_{\left|q-q^{\prime}\right| \leq 1}\left(\dot{S}_{q^{\prime}-1}-\dot{S}_{q-1}\right) u \cdot \nabla \dot{\Delta}_{q} \dot{\Delta}_{q^{\prime}} \theta+\sum_{\left|q-q^{\prime}\right| \leq 4}\left[\dot{\Delta}_{q}, \dot{S}_{q^{\prime}-1} u\right] \cdot \nabla \dot{\Delta}_{q^{\prime}} \theta .
$$

D'après la proposition 4.6 , on a

$$
\left\|\dot{\Delta}_{q} \theta(t)\right\|_{L^{p}} \leq\left\|\dot{\Delta}_{q} \theta_{0}\right\|_{L^{p}}+\int_{0}^{t}\left\|\dot{\Delta}_{q} f(\tau)\right\|_{L^{p}} d \tau+\int_{0}^{t}\left\|F_{q}(\tau)\right\|_{L^{p}} d \tau .
$$

Il s'agit maintenant de majorer $\left\|F_{q}\right\|_{L^{p}}$ de façon adéquate. Le premier terme se traite en faisant appel à la proposition 3.14. Comme $s<1+\frac{N}{p_{1}}$, on obtient

$$
\left\|\dot{\Delta}_{q} \dot{T}_{\partial_{i} \theta} u^{i}\right\|_{L^{p}} \leq C 2^{-q s} a_{q}\|\nabla u\|_{\dot{B}_{p_{1}, 1}^{\frac{N}{p_{1}}}}\|\theta\|_{\dot{B}_{p, r}^{s}} \quad \text { avec } \quad\left\|a_{q}\right\|_{\ell^{r}} \leq 1 .
$$

De même, sous la condition $s+1+\min \left(\frac{N}{p_{1}}, \frac{N}{p^{\prime}}\right)>0$, la proposition 3.14 donne

$$
\left\|\partial_{i} \dot{\Delta}_{q} \dot{R}\left(u^{i}, \theta\right)\right\|_{L^{p}} \leq C a_{q} 2^{-q s}\|\nabla u\|_{\dot{B}_{p_{1}, 1}^{\frac{N}{p_{1}}}}\|\theta\|_{\dot{B}_{p, r}^{s}} \quad \text { avec } \quad\left\|a_{q}\right\|_{\ell^{r}} \leq 1,
$$


et dans le cas limite $s+1+\left(\frac{N}{p_{1}}, \frac{N}{p^{\prime}}\right)=0$,

$$
\sup _{q \in \mathbb{Z}}\left\|\partial_{i} \dot{\Delta}_{q} \dot{R}\left(u^{i}, \theta\right)\right\|_{L^{p}} \leq C 2^{-q s}\|\nabla u\|_{\dot{B}_{p_{1}, 1}^{\frac{N}{p_{1}}}}\|\theta\|_{\dot{B}_{p, \infty}^{s}} .
$$

Le troisième terme de $F_{q}$ est une fonction localisée spectralement dans une couronne dyadique $2^{q} \mathcal{C}$. Ainsi, sans aucune restriction sur $s$, on a

$$
\sum_{\left|q-q^{\prime}\right| \leq 1}\left\|\left(\dot{S}_{q-1}-\dot{S}_{q^{\prime}-1}\right) u \cdot \nabla \dot{\Delta}_{q} \dot{\Delta}_{q^{\prime}} \theta\right\|_{L^{p}} \leq C a_{q} 2^{-q s}\|\nabla u\|_{\dot{B}_{p_{1}, 1}^{\frac{N}{p_{1}}}}\|\theta\|_{\dot{B}_{p, r}^{s}} .
$$

Enfin, par une inégalité classique sur les commutateurs (voir par exemple [6]), on a

$$
\left\|\left[\dot{\Delta}_{q}, \dot{S}_{q^{\prime}-1} u\right] \cdot \nabla \dot{\Delta}_{q^{\prime}} \theta\right\|_{L^{p}} \lesssim 2^{-q}\left\|\nabla \dot{S}_{q^{\prime}-1} u\right\|_{L^{\infty}}\left\|\dot{\Delta}_{q^{\prime}} \nabla \theta\right\|_{L^{p}}
$$

En conséquence,

$$
\left\|\left[\dot{\Delta}_{q}, \dot{S}_{q^{\prime}-1} u\right] \cdot \nabla \dot{\Delta}_{q^{\prime}} \theta\right\|_{L^{p}} \lesssim 2^{q^{\prime}-q}\|\nabla u\|_{L^{\infty}}\left\|\dot{\Delta}_{q^{\prime}} \theta\right\|_{L^{p}},
$$

d'où

$\sum_{\left|q-q^{\prime}\right| \leq 4}\left\|\left[\dot{\Delta}_{q}, \dot{S}_{q^{\prime}-1} u\right] \cdot \nabla \dot{\Delta}_{q^{\prime}} \theta\right\|_{L^{p}} \leq C 2^{-q s} a_{q}\|\nabla u\|_{L^{\infty}}\|\theta\|_{\dot{B}_{p, r}^{s}} \quad$ avec $\quad\left\|\left(a_{q}\right)\right\|_{\ell^{r}} \leq 1$.

En sommant toutes ces estimations et en utilisant le fait que $\dot{B}_{p_{1}, 1}^{\frac{N}{p_{1}}} \hookrightarrow L^{\infty}$, on trouve (en supposant $r=\infty$ dans le cas limite) :

$$
\left\|F_{q}\right\|_{L^{p}} \lesssim 2^{-q s} a_{q}\|\nabla u\|_{\dot{B}_{p_{1}, 1}^{\frac{N}{p_{1}}}}\|\theta\|_{\dot{B}_{p, r}^{s}} \quad \text { avec } \quad\left\|a_{q}\right\|_{\ell^{r}} \leq 1
$$

Il ne reste plus qu'à injecter cette inégalité dans (31), à multiplier par $2^{q s}$ puis à prendre la norme $\ell^{r}(\mathbb{Z})$ en $q$ de chaque membre. On obtient

$$
\|\theta\|_{\widetilde{L}_{t}^{\infty}\left(\dot{B}_{p, r}^{s}\right)} \leq\left\|\theta_{0}\right\|_{\dot{B}_{p, r}^{s}}+\|f\|_{\widetilde{L}_{t}^{1}\left(\dot{B}_{p, r}^{s}\right)}+C \int_{0}^{t}\|\nabla u(\tau)\|_{\dot{B}_{p_{1}, 1}^{\frac{N}{p_{1}}}}\|\theta(\tau)\|_{\dot{B}_{p, r}^{s}} d \tau
$$

et le lemme de Gronwall permet alors d'obtenir l'estimation souhaitée.

La présence du terme exponentiel dans l'estimation a priori de la proposition 4.7 semble ôter tout espoir de démontrer des résultats globaux pour le système de Boussinesq.

Dans [31] cependant, M. Vishik a remarqué que cette croissance exponentielle n'a pas lieu (dans le cas $f \equiv 0$ ) si l'on travaille dans des espaces de Besov à indice de régularité nul. Le résultat de $\mathrm{M}$. Vishik a été redémontré récemment par T. Hmidi et S. Keraani par une méthode plus robuste qui permet entre autres d'ajouter un terme de diffusion dans l'équation de transport (voir [18]). Nous utiliserons la version suivante du résultat de Vishik :

TOME $136-2008-\mathrm{N}^{\mathrm{O}} 2$ 
Proposition 4.8. - Soit $\theta \in \widetilde{L}_{T}^{\infty}\left(\dot{B}_{p, r}^{0}\right)$ une solution de (27) avec $f \in$ $\widetilde{L}_{T}^{1}\left(\dot{B}_{p, r}^{0}\right)$ et $\nabla u \in L^{1}\left([0, T], \dot{B}_{\infty, 1}^{0}\right)$ à divergence nulle. Alors on a la majoration suivante pour tout $t \in[0, T]$ :

$$
\|\theta\|_{\widetilde{L}_{t}^{\infty}\left(\dot{B}_{p, r}^{0}\right)} \leq C\left(\left\|\theta_{0}\right\|_{\dot{B}_{p, r}^{0}}+\|f\|_{\widetilde{L}_{t}^{1}\left(\dot{B}_{p, r}^{0}\right)}\right)\left(1+\int_{0}^{t}\|\nabla u(s)\|_{\dot{B}_{\infty, 1}^{0}} d s\right) .
$$

Démonstration. - Nous adoptons l'approche utilisée dans [18]. Pour simplifier la présentation, on se limite au cas $r=1$ (qui est le seul utilisé dans l'article).

Tout d'abord, remarquons que par unicité de la solution de l'équation de transport, on a $\theta=\sum_{q \in \mathbb{Z}} \theta_{q}$ où $\theta_{q}$ est la solution de l'équation

$$
\left\{\begin{array}{l}
\partial_{t} \theta_{q}+u \cdot \nabla \theta_{q}=\dot{\Delta}_{q} f \\
\theta_{q}(0, x)=\dot{\Delta}_{q} \theta_{0}(x)
\end{array}\right.
$$

On a clairement

$$
\|\theta\|_{\dot{B}_{p, 1}^{0}} \leq \sum_{j, q \in \mathbb{Z}}\left\|\dot{\Delta}_{j} \theta_{q}\right\|_{L^{p}}=\sum_{|j-q| \leq Q}\left\|\dot{\Delta}_{j} \theta_{q}\right\|_{L^{p}}+\sum_{|j-q| \geq Q}\left\|\dot{\Delta}_{j} \theta_{q}\right\|_{L^{p}},
$$

où $Q$ est un paramètre entier que l'on ajustera plus loin.

En faisant appel à la proposition 4.6, on constate que

$$
\begin{aligned}
\sum_{|j-q| \leq Q}\left\|\dot{\Delta}_{j} \theta_{q}(t)\right\|_{L^{p}} & \lesssim \sum_{|j-q| \leq Q}\left\|\theta_{q}(t)\right\|_{L^{p}}, \\
& \lesssim Q \sum_{q}\left(\left\|\dot{\Delta}_{q} \theta_{0}\right\|_{L^{p}}+\left\|\dot{\Delta}_{q} f\right\|_{L_{t}^{1}\left(L^{p}\right)}\right) \\
& \lesssim Q\left(\left\|\theta_{0}\right\|_{\dot{B}_{p, 1}^{0}}+\|f\|_{L_{t}^{1}\left(\dot{B}_{p, 1}^{0}\right)}\right) .
\end{aligned}
$$

Pour traiter la deuxième somme de (35), on utilise la proposition 4.7 avec donnée initiale $\dot{\Delta}_{q} \theta_{0}$ dans $\dot{B}_{p, 1}^{ \pm \varepsilon}$ et terme de force dans $L^{1}\left(0, T ; \dot{B}_{p, 1}^{ \pm \varepsilon}\right)$ (avec $\varepsilon$ choisi dans $(0,1))$. On peut donc écrire

$$
\left\|\theta_{q}(t)\right\|_{\dot{B}_{p, 1}^{ \pm \varepsilon}} \leq\left(\left\|\dot{\Delta}_{q} \theta_{0}\right\|_{\dot{B}_{p, 1}^{ \pm \varepsilon}}+\left\|\dot{\Delta}_{q} f\right\|_{L_{t}^{1}\left(\dot{B}_{p, 1}^{ \pm \varepsilon}\right)}\right) \exp \left(C \int_{0}^{t}\|\nabla u\|_{\dot{B}_{\infty, 1}^{0}} d \tau\right),
$$

ce qui implique

$$
\left\|\dot{\Delta}_{j} \theta_{q}(t)\right\|_{L^{p}} \leq 2^{-\varepsilon|q-j|} a_{j}\left(\left\|\dot{\Delta}_{q} \theta_{0}\right\|_{L^{p}}+\left\|\dot{\Delta}_{q} f\right\|_{L_{t}^{1}\left(L^{p}\right)}\right) \exp \left(C \int_{0}^{t}\|\nabla u\|_{\dot{B}_{\infty, 1}^{0}} d \tau\right),
$$

avec $\left\|\left(a_{j}\right)\right\|_{\ell^{1}(\mathbb{Z})} \leq 1$. Par sommation sur les indices $(j, q)$ tels que $|j-q| \geq Q$, on trouve

$$
\sum_{|j-q| \geq Q}\left\|\dot{\Delta}_{j} \theta_{q}(t)\right\|_{L^{p}} \leq 2^{-Q \varepsilon} C\left(\left\|\theta_{0}\right\|_{\dot{B}_{p, 1}^{0}}+\|f\|_{L_{t}^{1}\left(\dot{B}_{p, 1}^{0}\right)}\right) \exp \left(C \int_{0}^{t}\|\nabla u\|_{\dot{B}_{\infty, 1}^{0}} d \tau\right) .
$$

Il ne reste plus qu'à choisir $Q$ tel que $Q \varepsilon \log 2 \approx\left(1+C \int_{0}^{t}\|\nabla u\|_{\dot{B}_{\infty, 1}^{0}} d \tau\right)$. 
4.4. Estimations à pertes. - La démonstration de l'unicité pour le système de Boussinesq en dimension deux repose sur des estimations a priori avec perte de régularité pour les équations de transport-diffusion du type

$$
\left\{\begin{array}{l}
\partial_{t} \rho+\operatorname{div}(\rho u)-\nu \Delta \rho=f \\
\left.\rho\right|_{t=0}=\rho_{0}
\end{array}\right.
$$

avec un champ de vecteurs $u$ à divergence nulle qui n'est pas lipschitzien en espace.

Dans le cas sans diffusion, H. Bahouri et J.-Y. Chemin ont remarqué (voir [2]) que si le champ de vitesses était localement intégrable en temps à valeurs log-lipschitz, i.e.

$$
|u(t, x)-u(t, y)| \leq \gamma(t)|x-y|(1-\log |x-y|)
$$

pour tout $|x-y|<1$ avec $\gamma \in L_{\text {loc }}^{1}(0, T)$, alors la régularité de la donnée initiale était susceptible de se dégrader au cours du temps. Par exemple, si $\rho_{0}$ appartient à l'espace de Sobolev $H^{s}$ alors la solution à l'instant $t$ est seulement dans $H^{s-V(t)}$ avec $V(t)=C \int_{0}^{t} \gamma(\tau) d \tau$.

Nous souhaitons démontrer des estimations analogues valables pour tout $\nu \geq 0$, sous des hypothèses légèrement plus générales que celles de [2] et avec en sus un gain de régularité de type parabolique si $\nu>0$.

Nous avons opté pour un cadre fonctionnel non homogène. De ce fait, nous ferons appel à des blocs dyadiques non homogènes définis comme suit :

$$
\Delta_{q}:=\dot{\Delta}_{q} \quad \text { si } \quad q \geq 0, \quad \Delta_{-1}:=\dot{S}_{0} \quad \text { et } \quad \Delta_{q}:=0 \quad \text { si } \quad q \leq-2,
$$

et à la troncature basses fréquences

$$
S_{q}:=\sum_{p \leq q-1} \Delta_{p}
$$

Proposition 4.9. - Soit $s \in]-1-\frac{N}{2}, 1+\frac{N}{2}$ [ et $\rho$ une solution de l'équation de transport-diffusion (36). Il existe $N_{0} \in \mathbb{N}$ ne dépendant que du support de la fonction $\varphi$ intervenant dans la décomposition de Littlewood-Paley, une constante universelle $C_{0}$ et deux constantes $c$ et $C$ ne dépendant que de $s$ et de $N$ tels que si

$$
\|u\|_{\widetilde{L}_{T}^{1}\left(B_{2, \infty}^{1+\frac{N}{2}}\right)} \leq c
$$

alors on a l'estimation a priori suivante pour tout $t \in[0, T]$ :

$$
\begin{aligned}
\sup _{\substack{q \geq-1 \\
\tau \in[0, t]}} 2^{q s-\varepsilon_{q}(\tau)}\left\|\Delta_{q} \rho(\tau)\right\|_{L^{2}} & +\nu \sup _{q \geq 0} \int_{0}^{t} 2^{q(s+2)-\varepsilon_{q}(\tau)}\left\|\Delta_{q} \rho(\tau)\right\|_{L^{2}} d \tau \\
& \leq C_{0}\left(\left\|\rho_{0}\right\|_{B_{2, \infty}^{s}}+\sup _{q \geq-1} \int_{0}^{t} 2^{q s-\varepsilon_{q}(\tau)}\left\|\Delta_{q} f(\tau)\right\|_{L^{2}} d \tau\right)
\end{aligned}
$$

TOME $136-2008-\mathrm{N}^{\mathrm{O}} 2$ 
ò̀ $\varepsilon_{q}(t):=C \sum_{q^{\prime}=-1}^{q} 2^{q^{\prime}\left(1+\frac{N}{2}\right)} \int_{0}^{t}\left\|\check{\Delta}_{q^{\prime}} u\right\|_{L^{2}} d \tau$ avec $\check{\Delta}_{q^{\prime}}=\sum_{|\alpha| \leq N_{0}} \Delta_{q^{\prime}+\alpha}$ vérifie

$$
\varepsilon_{q}(T)-\varepsilon_{q^{\prime}}(T) \leq \frac{1}{2}\left(1+\frac{N}{2}+s\right)\left(q-q^{\prime}\right) \quad \text { pour } \quad q \geq q^{\prime} .
$$

Démonstration. - La démonstration de cette proposition se trouve implicitement dans [13] (voir th. 3.12) mais l'effet régularisant du Laplacien n'y est pas explicité. Étant donné que l'exploitation de cet effet régularisant est la clef de la preuve de l'unicité pour le système de Boussinesq en dimension 2, nous donnons ici la démonstration intégrale de l'estimation a priori.

En appliquant l'opérateur $\Delta_{q}$ à (36) et en procédant comme dans la preuve de la proposition 4.7 , on obtient

$$
\partial_{t} \Delta_{q} \rho+S_{q-1} u \cdot \nabla \Delta_{q} \rho-\nu \Delta_{q} \rho=\Delta_{q} f+F_{q}^{1}+F_{q}^{2}+F_{q}^{3}+F_{q}^{4}
$$

avec

$$
\begin{aligned}
F_{q}^{1} & :=\sum_{\left|q^{\prime}-q\right| \leq 4}\left[S_{q^{\prime}-1} u, \Delta_{q}\right] \cdot \nabla \Delta_{q^{\prime}} \rho \\
F_{q}^{2} & :=\sum_{\left|q^{\prime}-q\right| \leq 1}\left(S_{q-1}-S_{q^{\prime}-1}\right) u \cdot \nabla \Delta_{q} \Delta_{q^{\prime}} \rho \\
F_{q}^{3} & :=-\Delta_{q}\left(\sum_{\left|q^{\prime}-q\right| \leq 4} S_{q^{\prime}-1} \partial_{i} \rho \Delta_{q^{\prime}} u^{i}\right) \\
F_{q}^{4} & :=-\sum_{q^{\prime} \geq q-3} \partial_{i} \Delta_{q}\left(\Delta_{q^{\prime}} \rho\left(\sum_{|\alpha| \leq 1} \Delta_{q^{\prime}+\alpha}\right) u^{i}\right) .
\end{aligned}
$$

Une méthode d'énergie standard combinée avec l'inégalité de Bernstein donne

$$
\frac{1}{2} \frac{d}{d t}\left\|\Delta_{q} \rho\right\|_{L^{2}}^{2}+\nu_{q} 2^{2 q}\left\|\Delta_{q} \rho\right\|_{L^{2}}^{2} \leq\left(\left\|\Delta_{q} f\right\|_{L^{2}}+\sum_{i=1}^{4}\left\|F_{q}^{i}\right\|_{L^{2}}\right)\left\|\Delta_{q} \rho\right\|_{L^{2}}
$$

avec $\nu_{q}:=\kappa \nu$ si $q \geq 0$ et $\nu_{-1}=0$, la constante $\kappa$ dépendant uniquement du support de $\varphi$.

Par des manipulations classiques, on a

$$
\begin{aligned}
& \left\|F_{q}^{1}\right\|_{L^{2}} \leq \sum_{\left|q^{\prime}-q\right| \leq 4}\left\|\nabla S_{q^{\prime}-1} u\right\|_{L^{\infty}}\left\|\Delta_{q^{\prime}} \rho\right\|_{L^{2}}, \\
& \left\|F_{q}^{2}\right\|_{L^{2}} \leq \sum_{\left|q^{\prime}-q\right| \leq 1} 2^{q}\left\|\check{\Delta}_{q} u\right\|_{L^{\infty}}\left\|\Delta_{q} \rho\right\|_{L^{2}}, \\
& \left\|F_{q}^{3}\right\|_{L^{2}} \leq \sum_{q^{\prime} \leq q+2} 2^{q^{\prime}}\left\|\Delta_{q^{\prime}} \rho\right\|_{L^{\infty}}\left\|\check{\Delta}_{q} u\right\|_{L^{2}}, \\
& \left\|F_{q}^{4}\right\|_{L^{2}} \leq \sum_{q^{\prime} \geq q-3} 2^{q\left(1+\frac{N}{2}\right)}\left\|\Delta_{q^{\prime}} \rho\right\|_{L^{2}}\left\|\check{\Delta}_{q^{\prime}} u\right\|_{L^{2}},
\end{aligned}
$$


en notant $\check{\Delta}_{q}=\sum_{|\alpha| \leq N_{0}} \Delta_{q+\alpha}$ avec $N_{0}$ suffisamment grand.

En utilisant le fait que

$$
\begin{gathered}
\left\|\nabla S_{q^{\prime}-1} u\right\|_{L^{\infty}} \lesssim \sum_{q^{\prime \prime}<q^{\prime}-N_{0}} 2^{q^{\prime \prime}\left(1+\frac{N}{2}\right)}\left\|\check{\Delta}_{q^{\prime \prime}} u\right\|_{L^{2}}, \\
\left\|\check{\Delta}_{q} u\right\|_{L^{\infty}} \lesssim 2^{q \frac{N}{2}}\left\|\check{\Delta}_{q} u\right\|_{L^{2}}, \quad\left\|\Delta_{q^{\prime}} \rho\right\|_{L^{\infty}} \lesssim 2^{q^{\prime} \frac{N}{2}}\left\|\Delta_{q^{\prime}} \rho\right\|_{L^{2}},
\end{gathered}
$$

et en injectant les majorations obtenues ci-dessus dans (38), on obtient

$$
\begin{aligned}
& \frac{1}{2} \frac{d}{d t}\left\|\Delta_{q} \rho\right\|_{L^{2}}^{2}+\nu_{q} 2^{2 q}\left\|\Delta_{q} \rho\right\|_{L^{2}}^{2} \leq\left(\left\|\Delta_{q} f\right\|_{L^{2}}\right. \\
& \left.+C \sum_{q^{\prime} \geq q-4} 2^{\left(q-q^{\prime}\right)\left(1+\frac{N}{2}\right)} \varepsilon_{q^{\prime}}^{\prime}\left\|\Delta_{q^{\prime}} \rho\right\|_{L^{2}}+C \sum_{q^{\prime} \leq q} 2^{\left(q^{\prime}-q\right)\left(1+\frac{N}{2}\right)} \varepsilon_{q}^{\prime}\left\|\Delta_{q^{\prime}} \rho\right\|_{L^{2}}\right)\left\|\Delta_{q} \rho\right\|_{L^{2}}
\end{aligned}
$$

$\operatorname{avec} \varepsilon_{p}(t):=\int_{0}^{t} \sum_{p^{\prime} \leq p} 2^{p^{\prime}\left(1+\frac{N}{2}\right)}\left\|\check{\Delta}_{p} u\right\|_{L^{2}} d \tau$.

Pour $\lambda>0$, on pose

$$
\rho_{q}^{\lambda}(t):=2^{q s} e^{-\lambda \varepsilon_{q}(t)}\left\|\Delta_{q} \rho(t)\right\|_{L^{2}} \quad \text { et } \quad f_{q}^{\lambda}(t):=2^{q s} e^{-\lambda \varepsilon_{q}(t)}\left\|\Delta_{q} f(t)\right\|_{L^{2}}
$$

de telle sorte que

$$
\begin{aligned}
& \frac{1}{2} \frac{d}{d t}\left(\rho_{q}^{\lambda}\right)^{2}+\lambda \varepsilon_{q}^{\prime}\left(\rho_{q}^{\lambda}\right)^{2}+\nu_{q} 2^{2 q}\left(\rho_{q}^{\lambda}\right)^{2} \leq \rho_{q}^{\lambda}\left(f_{q}^{\lambda}\right. \\
& \left.+C 2^{q s} e^{-\lambda \varepsilon_{q}}\left(\sum_{q^{\prime} \geq q-4} 2^{\left(q-q^{\prime}\right)\left(1+\frac{N}{2}\right)} \varepsilon_{q^{\prime}}^{\prime}\left\|\Delta_{q^{\prime}} \rho\right\|_{L^{2}}+\sum_{q^{\prime} \leq q} 2^{\left(q^{\prime}-q\right)\left(1+\frac{N}{2}\right)} \varepsilon_{q}^{\prime}\left\|\Delta_{q^{\prime}} \rho\right\|_{L^{2}}\right)\right) .
\end{aligned}
$$

De l'inégalité précédente, on déduit après intégration en temps :

$$
\begin{gathered}
\rho_{q}^{\lambda}(t)+\nu_{q} 2^{2 q} \int_{0}^{t} \rho_{q}^{\lambda}(\tau) d \tau+\lambda \int_{0}^{t} \varepsilon_{q}^{\prime}(\tau) \rho_{q}^{\lambda}(\tau) d \tau \leq \rho_{q}^{\lambda}(0)+\int_{0}^{t} f_{q}^{\lambda}(\tau) d \tau \\
+C \sum_{q^{\prime} \geq q-4} 2^{\left(q-q^{\prime}\right)\left(1+\frac{N}{2}+s\right)} \int_{0}^{t} \varepsilon_{q^{\prime}}^{\prime}(\tau) e^{\lambda\left(\varepsilon_{q^{\prime}}(\tau)-\varepsilon_{q}(\tau)\right)} \rho_{q^{\prime}}^{\lambda}(\tau) d \tau \\
+C \sum_{q^{\prime} \leq q} 2^{\left(q^{\prime}-q\right)\left(1+\frac{N}{2}-s\right)} \int_{0}^{t} \varepsilon_{q}^{\prime}(\tau) e^{\lambda\left(\varepsilon_{q^{\prime}}(\tau)-\varepsilon_{q}(\tau)\right)} \rho_{q^{\prime}}^{\lambda}(\tau) d \tau
\end{gathered}
$$

En écrivant $\varepsilon_{q}^{\prime}(\tau)=\varepsilon_{q^{\prime}}^{\prime}(\tau)+\left(\varepsilon_{q}^{\prime}-\varepsilon_{q^{\prime}}^{\prime}\right)(\tau)$ dans le dernier terme et en remarquant que $\left(\varepsilon_{n}\right)_{n \geq-1}$ est une suite croissante de fonctions croissantes positives, on 
obtient pour tout $q \geq-1$,

$$
\begin{gathered}
\rho_{q}^{\lambda}(t)+\nu_{q} 2^{2 q} \int_{0}^{t} \rho_{q}^{\lambda}(\tau) d \tau+\lambda \int_{0}^{t} \varepsilon_{q}^{\prime}(\tau) \rho_{q}^{\lambda}(\tau) d \tau \leq \rho_{q}^{\lambda}(0)+\int_{0}^{t} f_{q}^{\lambda}(\tau) d \tau \\
+C \sum_{q^{\prime} \geq q} 2^{\left(q-q^{\prime}\right)\left(1+\frac{N}{2}+s\right)} e^{\lambda\left(\varepsilon_{q^{\prime}}(t)-\varepsilon_{q}(t)\right)} \int_{0}^{t} \varepsilon_{q^{\prime}}^{\prime}(\tau) \rho_{q^{\prime}}^{\lambda}(\tau) d \tau \\
+C \sum_{q^{\prime} \leq q} 2^{\left(q^{\prime}-q\right)\left(1+\frac{N}{2}-s\right)} \int_{0}^{t} \varepsilon_{q^{\prime}}^{\prime}(\tau) \rho_{q^{\prime}}^{\lambda}(\tau) d \tau+\frac{C}{\lambda} \sum_{q^{\prime} \leq q} 2^{\left(q^{\prime}-q\right)\left(1+\frac{N}{2}-s\right)} \sup _{\tau \in[0, t]} \rho_{q^{\prime}}^{\lambda}(\tau) .
\end{gathered}
$$

Supposons désormais que

$$
\sup _{q \geq-1} 2^{q\left(1+\frac{N}{2}\right)}\left\|\check{\Delta}_{q} u\right\|_{L_{t}^{1}\left(L^{2}\right)} \leq \varepsilon \log 2 \text { pour un } \varepsilon \text { tel que } \lambda \varepsilon \leq \frac{1}{2}\left(\frac{N}{2}+1+s\right)
$$

de telle sorte que pour tout $q^{\prime} \geq q \geq-1$, on ait

$$
2^{\left(q-q^{\prime}\right)\left(1+\frac{N}{2}+s\right)} e^{\lambda\left(\varepsilon_{q^{\prime}}(t)-\varepsilon_{q}(t)\right)} \leq 2^{\frac{q-q^{\prime}}{2}\left(1+\frac{N}{2}+s\right)} .
$$

Alors en prenant la borne supérieure en $q$ de l'inégalité précédente, on obtient

$$
\begin{aligned}
\sup _{\substack{q \geq-1 \\
\tau \in[0, t]}}\left(\rho_{q}^{\lambda}(\tau)+\right. & \left.\nu_{q} 2^{2 q} \int_{0}^{\tau} \rho_{q}^{\lambda}\left(\tau^{\prime}\right) d \tau^{\prime}+\lambda \int_{0}^{\tau} \varepsilon_{q}^{\prime}\left(\tau^{\prime}\right) \rho_{q}^{\lambda}\left(\tau^{\prime}\right) d \tau^{\prime}\right) \leq \sup _{q \geq-1} \rho_{q}^{\lambda}(0) \\
& +\sup _{q \geq-1} \int_{0}^{t} f_{q}^{\lambda}(\tau) d \tau+C \sup _{q \geq-1} \int_{0}^{t} \varepsilon_{q}^{\prime}(\tau) \rho_{q}^{\lambda}(\tau) d \tau+\frac{C}{\lambda} \sup _{\substack{q \geq-1 \\
\tau \in[0, t]}} \rho_{q}^{\lambda}(\tau) .
\end{aligned}
$$

En conséquence, on a,

$$
\begin{aligned}
& \sup _{\substack{q \geq-1 \\
\tau \in[0, t]}} \rho_{q}^{\lambda}(\tau)+\nu \sup _{q \geq 0} 2^{2 q} \int_{0}^{t} \rho_{q}^{\lambda}(\tau) d \tau+\lambda \sup _{q \geq-1} \int_{0}^{t} \varepsilon_{q}^{\prime}(\tau) \rho_{q}^{\lambda}(\tau) d \tau \leq 3\left\|\rho_{0}\right\|_{B_{2, \infty}^{s}} \\
& +3 \sup _{q \geq-1} \int_{0}^{t} f_{q}^{\lambda}(\tau) d \tau+3 C \sup _{q \geq-1} \int_{0}^{t} \varepsilon_{q^{\prime}}^{\prime}(\tau) \rho_{q^{\prime}}^{\lambda}(\tau) d \tau+\frac{3 C}{\lambda} \sup _{\substack{q \geq-1 \\
\tau \in[0, t]}} \rho_{q}^{\lambda}(\tau) .
\end{aligned}
$$

Il ne reste plus qu'à choisir $\lambda=6 C$ pour conclure à l'inégalité souhaitée :

$$
\sup _{\substack{q \geq-1 \\ \tau \in[0, t]}} \rho_{q}^{\lambda}(\tau)+\nu \sup _{q \geq 0} 2^{2 q} \int_{0}^{t} \rho_{q}^{\lambda}(\tau) d \tau \leq C_{0}\left(\left\|\rho_{0}\right\|_{B_{2, \infty}^{s}}+\sup _{q \geq-1} \int_{0}^{t} f_{q}^{\lambda}(\tau) d \tau\right) .
$$


Remarque 4.10. - L'estimation de la proposition 4.9 s'étend sans aucune difficulté au problème de Stokes non stationnaire avec convection :

$$
\left\{\begin{array}{l}
\partial_{t} \rho+\operatorname{div}(\rho u)-\nu \Delta \rho+\nabla \Pi=f, \\
\operatorname{div} u=0 .
\end{array}\right.
$$

La propriété de divergence nulle assure en effet que le terme $\nabla \Pi$ est «transparent » et que les estimations sur $\left\|\Delta_{q} \rho\right\|_{L^{2}}^{2}$ restent les mêmes que pour l'équation (36).

\section{Solutions fortes}

Cette partie est consacrée à la démonstration de résultats d'existence locale (ou globale à données petites) et d'unicité pour le système de Boussinesq.

5.1. Un résultat local. - Dans un premier temps, nous souhaitons établir le théorème 1.3. Pour cela, nous allons adopter le schéma classique suivant :

1. Construction d'une suite de solutions globales approchées,

2. Estimations uniformes en grande norme sur un (petit) intervalle de temps fixe,

3. Convergence en petite norme sur cet intervalle de temps,

4. Unicité.

Le critère de prolongement sera démontré à la fin de la section.

5.1.1. Construction d'une suite de solutions globales approchées. - Notons $\left(u_{L}, \nabla \Pi_{L}\right)$ la solution libre de l'équation de Stokes

$$
\partial_{t} u_{L}-\nu \Delta u_{L}+\nabla \Pi_{L}=0, \quad u_{L}(0)=u_{0} .
$$

On a bien sûr (cf remarque 3.12) :

$$
u_{L} \in \mathcal{C}_{b}\left(\mathbb{R}_{+} ; \dot{B}_{N, 1}^{0}\right) \cap L^{1}\left(\mathbb{R}_{+} ; \dot{B}_{N, 1}^{2}\right) \quad \text { et } \quad \nabla \Pi_{L} \in L^{1}\left(\mathbb{R}_{+} ; \dot{B}_{N, 1}^{0}\right) .
$$

On définit ensuite $\left(\theta^{0}, \bar{u}^{0}\right) \equiv\left(\theta_{0}, 0\right)$ puis on résout par récurrence les équations linéaires suivantes :

$$
\left\{\begin{array}{l}
\partial_{t} \theta^{n+1}+u^{n} \cdot \nabla \theta^{n+1}=0 \quad \text { avec } \quad u^{n}:=u_{L}+\bar{u}^{n} \\
\partial_{t} \bar{u}^{n+1}-\nu \Delta \bar{u}^{n+1}+\nabla \bar{\Pi}^{n+1}=-\operatorname{div}\left(u^{n} \otimes u^{n}\right)+\theta^{n+1} e_{N}, \\
\theta^{n+1}(0)=\theta_{0}, \quad u^{n+1}(0)=0 .
\end{array}\right.
$$

D'après la proposition 4.7, la remarque 3.12 et le fait que $\dot{B}_{N, 1}^{1}$ soit une algèbre, on obtient ainsi une suite $\left(\theta^{n}, \bar{u}^{n}, \nabla \bar{\Pi}^{n}\right)_{n \in \mathbb{N}}$ de solutions globales approchées vérifiant

$\theta^{n} \in \mathcal{C}\left(\mathbb{R}_{+} ; \dot{B}_{N, 1}^{0}\right), \quad \bar{u}^{n} \in \mathcal{C}\left(\mathbb{R}_{+} ; \dot{B}_{N, 1}^{0}\right) \cap L_{l o c}^{1}\left(\mathbb{R}_{+} ; \dot{B}_{N, 1}^{2}\right) \quad$ et $\quad \nabla \bar{\Pi}^{n} \in L_{l o c}^{1}\left(\mathbb{R}_{+} ; \dot{B}_{N, 1}^{0}\right)$. 
5.1.2. Estimations a priori uniformes en grande norme sur un intervalle de temps fixe.- D'après la proposition 4.8 , on a pour tout $t \geq 0$,

$$
\left\|\theta^{n+1}(t)\right\|_{\dot{B}_{N, 1}^{0}} \leq C\left\|\theta_{0}\right\|_{\dot{B}_{N, 1}^{0}}\left(1+\left\|u^{n}\right\|_{L_{t}^{1}\left(\dot{B}_{N, 1}^{2}\right)}\right) \text {. }
$$

Par ailleurs, d'après la remarque 3.12 et les lois de produit dans les espaces de Besov énoncées après la proposition 3.14, on a

$$
\begin{aligned}
\bar{U}^{n+1}(t):=\left\|\bar{u}^{n+1}\right\|_{L_{t}^{1}\left(\dot{B}_{N, 1}^{0}\right)}+\nu\left\|\bar{u}^{n+1}\right\|_{L_{t}^{1}\left(\dot{B}_{N, 1}^{2}\right)} & +\left\|\nabla \bar{\Pi}^{n+1}\right\|_{L_{t}^{1}\left(\dot{B}_{N, 1}^{0}\right)} \\
& \lesssim\left\|u^{n}\right\|_{L_{t}^{2}\left(\dot{B}_{N, 1}^{1}\right)}^{2}+\left\|\theta^{n+1}\right\|_{L_{t}^{1}\left(\dot{B}_{N, 1}^{0}\right)} .
\end{aligned}
$$

D'où, en insérant l'inégalité (41) dans l'inégalité ci-dessus,

$$
\begin{aligned}
\bar{U}^{n+1}(t) \leq C \nu^{-1} \bar{U}^{n}(t)\left(\bar{U}^{n}(t)\right. & \left.+t\left\|\theta_{0}\right\|_{\dot{B}_{N, 1}^{0}}\right) \\
& +C\left(\left\|u_{L}\right\|_{L_{t}^{2}\left(\dot{B}_{N, 1}^{1}\right)}^{2}+t\left\|\theta_{0}\right\|_{\dot{B}_{N, 1}^{0}}\left(1+\left\|u_{L}\right\|_{L_{t}^{1}\left(\dot{B}_{N, 1}^{2}\right)}\right)\right)
\end{aligned}
$$

avec $C \geq 1$ ne dépendant que de la dimension $N$.

On en déduit que $\bar{U}^{n}(t) \leq \nu /(2 C)$ tant que $t$ vérifie

$$
4 C^{2} \nu^{-1}\left(\left\|u_{L}\right\|_{L_{t}^{2}\left(\dot{B}_{N, 1}^{1}\right)}^{2}+t\left\|\theta_{0}\right\|_{\dot{B}_{N, 1}^{0}}\left(2+\left\|u_{L}\right\|_{L_{t}^{1}\left(\dot{B}_{N, 1}^{2}\right)}\right)\right) \leq 1,
$$

En revenant à (41) et en notant $T$ la borne supérieure des temps $t$ vérifiant (42), on peut finalement conclure que $\left(\theta^{n}, u^{n}, \nabla \Pi^{n}\right)_{n \in \mathbb{N}}$ est uniformément bornée dans l'espace $E_{T}$.

5.1.3. Convergence en petite norme. - Soit $\bar{\theta}^{n}:=\theta^{n}-\theta_{0}$. Nous allons montrer que, quitte à diminuer $T$ si nécessaire, la suite $\left(\bar{\theta}^{n}, \bar{u}^{n}, \nabla \bar{\Pi}^{n}\right)_{n \in \mathbb{N}}$ est de Cauchy dans l'espace

$$
F_{T}:=\mathcal{C}\left([0, T] ; \dot{B}_{N, 1}^{-1}\right) \times\left(\mathcal{C}\left([0, T] ; \dot{B}_{N, 1}^{-1}\right) \times L^{1}\left([0, T] ; \dot{B}_{N, 1}^{1}\right)\right)^{N} \times\left(L^{1}\left([0, T] ; \dot{B}_{N, 1}^{-1}\right)\right)^{N} .
$$

Comme les espaces de Besov utilisés sont homogènes, l'appartenance à $E_{T}$ n'entraîne a priori pas que $\left(\bar{\theta}^{n}, \bar{u}^{n}, \nabla \bar{\Pi}^{n}\right)$ est dans $F_{T}$. En s'aidant de (40) et de la proposition 3.14 , il n'est cependant pas difficile de vérifier que $\partial_{t} \bar{\theta}_{n}$ et $\partial_{t} \bar{u}_{n}$ sont dans $L^{2}\left(0, T ; \dot{B}_{N, 1}^{-1}\right)$. Sachant que $\bar{\theta}_{n}(0)$ et $\bar{u}_{n}(0)$ sont nuls, on peut alors facilement justifier que $\left(\bar{\theta}^{n}, \bar{u}^{n}, \nabla \bar{\Pi}^{n}\right)$ est dans $F_{T}$.

Notons $\delta \theta^{n}:=\theta^{n+1}-\theta^{n}, \delta u^{n}:=u^{n+1}-u^{n}$ et $\delta \Pi^{n}:=\Pi^{n+1}-\Pi^{n}$. Comme $\delta \theta^{n}$ vérifie

$$
\partial_{t} \delta \theta^{n}+u^{n-1} \cdot \nabla \delta \theta^{n}=-\operatorname{div}\left(\delta u^{n-1} \theta^{n}\right),
$$

la proposition 4.7 assure que

$$
\left\|\delta \theta^{n}(t)\right\|_{\dot{B}_{N, 1}^{-1}} \leq C e^{\left\|u^{n}\right\|_{L_{t}^{1}\left(\dot{B}_{N, 1}^{2}\right)}} \int_{0}^{t}\left\|\theta^{n} \delta u^{n-1}\right\|_{\dot{B}_{N, 1}^{0}} d \tau .
$$


Donc, grâce aux bornes uniformes obtenues dans l'étape précédente, on a

$$
\left\|\delta \theta^{n}(t)\right\|_{\dot{B}_{N, 1}^{-1}} \leq C_{T} \int_{0}^{t}\left\|\delta u^{n-1}\right\|_{\dot{B}_{N, 1}^{1},} d \tau
$$

avec $C_{T}$ indépendante de $n$ et de $t \in[0, T]$.

Ensuite, comme

$\partial_{t} \delta u^{n}-\nu \Delta \delta u^{n}+\nabla \delta \Pi^{n}=-\operatorname{div}\left(u^{n-1} \otimes \delta u^{n-1}\right)-\operatorname{div}\left(\delta u^{n-1} \otimes u^{n}\right)+\delta \theta^{n} e_{N}$, on a, d'après la remarque 3.12 ,

$$
\begin{aligned}
\delta U^{n}(t):= & \left\|\delta u^{n}\right\|_{L_{t}^{\infty}\left(\dot{B}_{N, 1}^{-1}\right)}+\nu\left\|\delta u^{n}\right\|_{L_{t}^{1}\left(\dot{B}_{N, 1}^{1}\right)}+\left\|\nabla \delta \Pi^{n}\right\|_{L_{t}^{1}\left(\dot{B}_{N, 1}^{-1}\right)} \\
& \leq C \int_{0}^{t}\left(\left\|u^{n-1} \otimes \delta u^{n-1}\right\|_{\dot{B}_{N, 1}^{0}}+\left\|\delta u^{n-1} \otimes u^{n}\right\|_{\dot{B}_{N, 1}^{0}}+\left\|\delta \theta^{n}\right\|_{\dot{B}_{N, 1}^{-1}}\right) d \tau .
\end{aligned}
$$

Le dernier terme peut être borné grâce à (43). En utilisant les lois de produit dans les espaces de Besov énoncées après la proposition 3.14, et les inégalités d'interpolation de la proposition 3.8 , on obtient

$$
\begin{aligned}
\left\|u^{n-1} \otimes \delta u^{n-1}\right\|_{L_{t}^{1}\left(\dot{B}_{N, 1}^{0}\right)} & +\left\|\delta u^{n-1} \otimes u^{n}\right\|_{L_{t}^{1}\left(\dot{B}_{N, 1}^{0}\right)} \\
& \leq C\left(\left\|u^{n-1}\right\|_{L_{t}^{2}\left(\dot{B}_{N, 1}^{1}\right)}+\left\|u^{n}\right\|_{L_{t}^{2}\left(\dot{B}_{N, 1}^{1}\right)}\right)\left\|\delta u^{n-1}\right\|_{L_{t}^{2}\left(\dot{B}_{N, 1}^{0}\right)} .
\end{aligned}
$$

En combinant avec (43), on a donc finalement

$$
\delta U^{n}(t) \leq \delta U^{n-1}(t) \nu^{-1 / 2}\left(C_{T} t+C\left(\left\|u^{n-1}\right\|_{L_{t}^{2}\left(\dot{B}_{N, 1}^{1}\right)}+\left\|u^{n}\right\|_{L_{t}^{2}\left(\dot{B}_{N, 1}^{1}\right)}\right)\right) .
$$

D'après (42), il existe une constante $c$ (que l'on peut choisir aussi petite que l'on veut quitte à diminuer $T$ ) telle que

$$
\left\|u^{n-1}\right\|_{L_{T}^{2}\left(\dot{B}_{N, 1}^{1}\right)}+\left\|u^{n}\right\|_{L_{T}^{2}\left(\dot{B}_{N, 1}^{1}\right)} \leq c \sqrt{\nu} .
$$

On en conclut que pour $T$ suffisamment petit, on a

$$
\forall t \in[0, T], \forall n \in \mathbb{N}, \delta U^{n}(t) \leq \frac{1}{2} \delta U^{n-1}(t) .
$$

La suite considérée est donc de Cauchy dans $F_{T}$.

5.1.4. Fin de la preuve de l'existence locale. - On a donc d'une part $\left(\bar{\theta}^{n}, \bar{u}^{n}, \nabla \bar{\Pi}^{n}\right)$ qui converge dans l'espace $F_{T}$ vers une fonction $(\bar{\theta}, \bar{u}, \nabla \bar{\Pi})$, et d'autre part les estimations uniformes en grande norme de la première étape. Cela permet d'affirmer que $\left(\theta^{n}, u^{n}, \nabla \Pi^{n}\right)_{n \in \mathbb{N}}$ converge vers une solution

$$
\left(\theta:=\bar{\theta}+\theta_{0}, u:=\bar{u}+u_{L}, \nabla \Pi:=\nabla\left(\Pi_{L}+\bar{\Pi}\right)\right)
$$

du système de Boussinesq appartenant à

$$
L_{T}^{\infty}\left(\dot{B}_{N, 1}^{0}\right) \times\left(L_{T}^{\infty}\left(\dot{B}_{N, 1}^{0}\right) \cap L_{T}^{1}\left(\dot{B}_{N, 1}^{2}\right)\right)^{N} \times\left(L_{T}^{1}\left(\dot{B}_{N, 1}^{0}\right)\right)^{N}
$$


La continuité en temps s'obtient à l'aide de la proposition 4.7 et de la remarque 3.12 en remarquant que (44) entraîne que $\partial_{t} \theta+\operatorname{div}(\theta u)$ et $\partial_{t} u-\nu \Delta u+\nabla \Pi$ sont dans $L_{T}^{1}\left(\dot{B}_{N, 1}^{0}\right)$.

5.1.5. Unicité. - L'unicité s'obtient par les mêmes arguments que la convergence en petite norme. Les détails sont laissés au lecteur.

5.1.6. Critère de prolongement. - Sous les hypothèses du théorèmes, on constate que $\partial_{t} \theta+u \cdot \nabla \theta$ et $\partial_{t} u-\nu \Delta u+\nabla \Pi$ sont dans $L^{1}\left(0, T^{*} ; \dot{B}_{N, 1}^{0}\right)$. D'après la proposition 4.7 et la remarque 3.12 , les fonctions $\theta$ et $u$ sont donc en fait dans $\widetilde{L}_{T}^{\infty}\left(\dot{B}_{N, 1}^{0}\right)$. En partant de n'importe que temps $t \in\left[0, T^{*}\right)$, on peut alors, grâce à (42) obtenir l'existence d'une solution avec données $(\theta(t), u(t))$ sur un intervalle de temps indépendant de $t$. Combiné avec la propriété d'unicité, cela permet de prolonger $(u, \theta)$ au-delà de $T^{*}$.

Cela achève la démonstration du théorème 1.3.

5.2. Résultats globaux à données petites. - Cette section est consacrée à la démonstration du théorème 1.4. Pour alléger la présentation, on convient désormais que $L^{\frac{N}{3}, \infty}$ désigne $L^{1}$ si $N=3$.

5.2.1. Unicité. — La partie unicité du théorème 1.4 découle de la proposition suivante :

Proposition 5.1. - Soit $\left(\theta_{1}, u_{1}, \nabla \Pi_{1}\right)$ et $\left(\theta_{2}, u_{2}, \nabla \Pi_{2}\right)$ deux solutions du système de Boussinesq telles que $\left(\theta_{1}, u_{1}\right)$ et $\left(\theta_{2}, u_{2}\right)$ appartiennent à l'espace

$$
\begin{aligned}
\left(\mathcal{C}\left([0, T] ; \dot{B}_{N, 1}^{0}\right) \cap L^{\infty}\left(0, T ; L^{\frac{N}{3}, \infty}\right)\right) \times & \\
& \left(\mathcal{C}\left([0, T] ; \dot{B}_{\infty, 1}^{-1}\right) \cap L^{1}\left(0, T ; \dot{B}_{\infty, 1}^{1}\right) \cap L^{\infty}\left(0, T ; L^{N, \infty}\right)\right)^{N} .
\end{aligned}
$$

Si les deux solutions coïncident à l'instant initial alors elles coïncident sur $[0, T]$.

Preuve. - Remarquons tout d'abord que du fait des hypothèses, on a

$$
\partial_{t} u_{i}-\mu \Delta u_{i} \in L_{T}^{1}\left(\dot{B}_{\infty, 1}^{-1}\right) \quad \text { pour } \quad i=1,2
$$

La proposition 3.11 assure donc que $u_{i} \in \widetilde{L}_{T}^{\infty}\left(\dot{B}_{\infty, 1}^{-1}\right)$. Ce fait sera utilisé à plusieurs reprises dans les calculs qui suivent.

Soit $(\delta \theta, \delta u, \delta \Pi)=\left(\theta_{1}-\theta_{2}, u_{1}-u_{2}, \Pi_{1}-\Pi_{2}\right)$. Remarquons que

$$
\left\{\begin{array}{l}
\partial_{t} \delta \theta+\operatorname{div}\left(u_{2} \delta \theta\right)=-\operatorname{div}\left(\delta u \theta_{1}\right), \\
\partial_{t} \delta u+\operatorname{div}\left(u_{2} \otimes \delta u\right)+\operatorname{div}\left(\delta u \otimes u_{1}\right)-\nu \Delta \delta u+\nabla \delta \Pi=\delta \theta e_{N} .
\end{array}\right.
$$


Admettons provisoirement que $(\delta \theta, \delta u, \nabla \delta \Pi)$ appartienne à l'espace $G_{T}:=L^{\infty}\left([0, T] ; \dot{B}_{N, \infty}^{-1}\right) \times\left(\mathcal{C}\left([0, T] ; \dot{B}_{N, \infty}^{-1}\right) \cap \widetilde{L}_{T}^{1}\left(\dot{B}_{N, \infty}^{1}\right)\right)^{N} \times\left(\widetilde{L}_{T}^{1}\left(\dot{B}_{N, \infty}^{-1}\right)\right)^{N}$.

On munit $G_{T}$ de la norme $\|(\theta, u, \nabla \Pi)\|_{G_{T}}:=\|\theta\|_{L_{T}^{\infty}\left(\dot{B}_{N, \infty}^{-1}\right)}+\|u\|_{L_{T}^{\infty}\left(\dot{B}_{N, \infty}^{-1}\right)}+\nu\|u\|_{\widetilde{L}_{T}^{1}\left(\dot{B}_{N, \infty}^{1}\right)}+\|\nabla \Pi\|_{\widetilde{L}_{T}^{1}\left(\dot{B}_{N, \infty}^{-1}\right)}$.

Pour montrer l'unicité dans $G_{T}$, nous allons suivre l'approche utilisée dans [14] : établir une inégalité différentielle sur $\|(\delta \theta, \delta u, \nabla \delta \Pi)\|_{G_{T}}$ grâce à un argument d'interpolation logarithmique puis conclure à l'aide du lemme d'Osgood.

Tout d'abord, en appliquant la proposition 4.7 et en utilisant (19), on obtient

$$
\|\delta \theta\|_{L_{t}^{\infty}\left(\dot{B}_{N, \infty}^{-1}\right)} \leq C e^{C\left\|u_{2}\right\|_{L_{t}^{1}\left(\dot{B}_{\infty, 1}^{1}\right)}} \int_{0}^{t}\left\|\delta u \theta_{1}\right\|_{\dot{B}_{N, \infty}^{0}} d \tau .
$$

Sachant que $\dot{B}_{N, 1}^{0} \hookrightarrow L^{N} \hookrightarrow \dot{B}_{N, \infty}^{0}$ et que $\dot{B}_{\infty, 1}^{0} \hookrightarrow L^{\infty}$, on peut écrire :

$$
\begin{aligned}
\left\|\delta u \theta_{1}\right\|_{\dot{B}_{N, \infty}^{0}} & \lesssim\|\delta u\|_{L^{\infty}}\left\|\theta_{1}\right\|_{L^{N}}, \\
& \lesssim\|\delta u\|_{\dot{B}_{\infty, 1}^{0}\left\|\theta_{1}\right\|_{\dot{B}_{N, 1}^{0}} .} .
\end{aligned}
$$

Cela permet de conclure que pour tout $t \leq T$, on a

$$
\|\delta \theta\|_{L_{t}^{\infty}\left(\dot{B}_{N, \infty}^{-1}\right)} \leq C e^{C\left\|u_{2}\right\|_{L_{t}^{1}\left(\dot{B}_{\infty, 1}^{1}\right)}}\|\delta u\|_{L_{t}^{1}\left(\dot{B}_{\infty, 1}^{0}\right)}\left\|\theta_{1}\right\|_{L_{t}^{\infty}\left(\dot{B}_{N, 1}^{0}\right)} .
$$

Pour majorer $\delta u$, on utilise la remarque 3.12 et l'on obtient pour une constante $C$ indépendante de $t$ :

$\delta U(t) \leq C\left(\left\|\operatorname{div}\left(u_{2} \otimes \delta u\right)\right\|_{\widetilde{L}_{t}^{1}\left(\dot{B}_{N, \infty}^{-1}\right)}+\left\|\operatorname{div}\left(\delta u \otimes u_{1}\right)\right\|_{\widetilde{L}_{t}^{1}\left(\dot{B}_{N, \infty}^{-1}\right)}+\|\delta \theta\|_{\widetilde{L}_{t}^{1}\left(\dot{B}_{N, \infty}^{-1}\right)}\right)$ avec $\delta U(t):=\|\delta u\|_{L_{t}^{\infty}\left(\dot{B}_{N, \infty}^{-1}\right)}+\|\delta u\|_{\widetilde{L}_{t}^{1}\left(\dot{B}_{N, \infty}^{1}\right)}+\|\nabla \delta \Pi\|_{\widetilde{L}_{t}^{1}\left(\dot{B}_{N, \infty}^{-1}\right)}$.

Les termes non linéaires se traitent à l'aide des décompositions de Bony suivantes :

$$
\begin{aligned}
& \operatorname{div}\left(u_{2} \otimes \delta u\right)=\dot{T}_{u_{2}^{j}} \partial_{j} \delta u+\partial_{j} \dot{R}\left(u_{2}^{j}, \delta u\right)+\dot{T}_{\partial_{j} \delta u} u_{2}^{j}, \\
& \operatorname{div}\left(\delta u \otimes u_{1}\right)=\dot{T}_{\partial_{j} u_{1}} \delta u^{j}+\partial_{j} \dot{R}\left(u_{1}, \delta u^{j}\right)+\partial_{j} \dot{T}_{\delta u^{j}} u_{1} .
\end{aligned}
$$

En utilisant les résultats de continuité de la proposition 3.14 (qui se généralisent de façon immédiate aux espaces $\widetilde{L}_{T}^{\rho}\left(\dot{B}_{p, r}^{s}\right)$ voir par exemple [12] ou [14]), et (19), on obtient les majorations

$$
\begin{aligned}
\left\|\operatorname{div}\left(u_{2} \otimes \delta u\right)\right\|_{\widetilde{L}_{t}^{1}\left(\dot{B}_{N, \infty}^{-1}\right)} & \lesssim\|\delta u\|_{\widetilde{L}_{t}^{2}\left(\dot{B}_{N, \infty}^{0}\right)}\left\|u_{1}\right\|_{\widetilde{L}_{t}^{2}\left(\dot{B}_{\infty, 1}^{0}\right)}, \\
\left\|\operatorname{div}\left(\delta u \otimes u_{1}\right)\right\|_{\widetilde{L}_{t}^{1}\left(\dot{B}_{N, \infty}^{-1}\right)} & \lesssim \int_{0}^{t}\|\delta u\|_{\dot{B}_{N, \infty}^{-1}}\left\|u_{1}\right\|_{\dot{B}_{\infty, 1}^{1}} d \tau .
\end{aligned}
$$


En conséquence, on a

$$
\delta U(t) \lesssim\left\|u_{2}\right\|_{\widetilde{L}_{t}^{2}\left(\dot{B}_{\infty, 1}^{0}\right)}\|\delta u\|_{\widetilde{L}_{t}^{2}\left(\dot{B}_{N, \infty}^{0}\right)}+\int_{0}^{t}\left(\|\delta \theta\|_{\dot{B}_{N, \infty}^{-1}}+\|\delta u\|_{\dot{B}_{N, \infty}^{-1}}\left\|u_{1}\right\|_{\dot{B}_{\infty, 1}^{1}}\right) d \tau .
$$

Le premier terme du membre de droite peut être absorbé à temps suffisamment petit. En appliquant l'inégalité de Gronwall, on obtient donc pour $t$ assez petit,

$$
\delta U(t) \leq C_{T} \int_{0}^{t}\|\delta \theta\|_{\dot{B}_{N, \infty}^{-1}} d \tau
$$

pour une constante $C_{T}$ ne dépendant que de normes de $u_{1}$ et $u_{2}$ sur $[0, T]$.

Par ailleurs, d'après un résultat d'interpolation logarithmique établi dans la proposition 1.8 de [12], on a

$$
\begin{aligned}
\|\delta u\|_{L_{t}^{1}\left(\dot{B}_{\infty, 1}^{0}\right)} & \lesssim\|\delta u\|_{\widetilde{L}_{t}^{1}\left(\dot{B}_{\infty, \infty}^{0}\right)} \log \left(e+\frac{\|\delta u\|_{L_{t}^{1}\left(\dot{B}_{\infty, 1}^{-1}\right)}+\|\delta u\|_{L_{t}^{1}\left(\dot{B}_{\infty, 1}^{1}\right)}}{\|\delta u\|_{\widetilde{L}_{t}^{1}\left(\dot{B}_{\infty, \infty}^{0}\right)}}\right), \\
& \lesssim\|\delta u\|_{\widetilde{L}_{t}^{1}\left(\dot{B}_{N, \infty}^{1}\right)} \log \left(e+\frac{\|\delta u\|_{L_{t}^{1}\left(\dot{B}_{\infty, 1}^{1}\right)}+t\|\delta u\|_{L_{t}^{\infty}\left(\dot{B}_{\infty, 1}^{-1}\right)}}{\|\delta u\|_{\widetilde{L}_{t}^{1}\left(\dot{B}_{N, \infty}^{1}\right)}}\right) .
\end{aligned}
$$

Par hypothèse, la fonction $V$ définie par

$$
V(t):=\|\delta u\|_{L_{t}^{1}\left(\dot{B}_{\infty, 1}^{1}\right)}+t\|\delta u\|_{L_{t}^{\infty}\left(\dot{B}_{\infty, 1}^{-1}\right)}
$$

est bornée sur $[0, T]$.

En injectant (46) dans (47), et en utilisant l'inégalité d'interpolation logarithmique, on obtient finalement compte tenu de la croissance de la fonction $x \longmapsto x \ln \left(e+\frac{y}{x}\right)$ et quitte à augmenter $C_{T}$,

$$
\delta U(t) \leq C_{T} \int_{0}^{t} \delta U(s) \log \left(e+\frac{V(s)}{\delta U(s)}\right) d s .
$$

Le lemme d'Osgood assure que $\delta U(t)=0$, et donc l'unicité sur un petit intervalle de temps. De l'unicité locale, on passe à l'unicité sur $[0, T]$ par des arguments classiques. $G_{T}$.

Il s'agit maintenant de justifier que $(\delta \theta, \delta u, \nabla \delta \Pi)$ appartient bien à l'espace

Vu les hypothèses, la fonction $\delta \theta$ appartient à $L^{\infty}\left([0, T] ; \dot{B}_{N, \infty}^{-1}\right)$. En effet, sachant que $L^{\frac{N}{3}, \infty} \hookrightarrow \dot{B}_{N, \infty}^{-2}\left(\right.$ cf lemme 3.9) et que $\theta_{i}$ appartient aussi à $L_{T}^{\infty}\left(\dot{B}_{N, 1}^{0}\right)$, on déduit par interpolation que

$$
\theta_{i} \in L_{T}^{\infty}\left(\dot{B}_{N, \infty}^{-1}\right) \quad \text { pour } \quad i=1,2 .
$$

Reste à justifier l'appartenance de $(\delta u, \nabla \delta \Pi)$ à

$$
\left(\mathcal{C}\left([0, T] ; \dot{B}_{N, \infty}^{-1}\right) \cap \widetilde{L}_{T}^{1}\left(\dot{B}_{N, \infty}^{1}\right)\right)^{N} \times\left(\widetilde{L}_{T}^{1}\left(\dot{B}_{N, \infty}^{-1}\right)\right)^{N}
$$


Pour cela, on peut décomposer $u_{i}$ (que l'on note dorénavant $u$ pour simplifier) en solution libre $u_{L}:=e^{\nu t \Delta} u_{0}$ et fluctuation $\bar{u}:=u-u_{L}$. On voit que $\bar{u}$ vérifie

$$
\left\{\begin{array}{l}
\partial_{t} \bar{u}-\nu \Delta \bar{u}=\mathcal{P}\left(\theta e_{N}-\operatorname{div}(u \otimes u)\right), \\
\bar{u}_{\mid t=0}=0 .
\end{array}\right.
$$

Tout d'abord, comme $u \in L_{T}^{2}\left(\dot{B}_{\infty, 1}^{0}\right)$ (par interpolation) et $\dot{B}_{\infty, 1}^{0} \hookrightarrow L^{\infty}$, et comme par ailleurs on a $u \in L_{T}^{\infty}\left(L^{N, \infty}\right)$, on obtient $u \in L_{T}^{2}\left(L^{2 N}\right)$. Cela assure que $u \otimes u \in L_{T}^{1}\left(L^{N}\right)$ puis, par injection et différentiation, que

$$
\operatorname{div}(u \otimes u) \in L_{T}^{1}\left(\dot{B}_{N, \infty}^{-1}\right) .
$$

En combinant (50), (48) avec (19), on constate donc que $\bar{u}$ est solution de l'équation de la chaleur avec donnée initiale nulle et terme de force dans $\widetilde{L}_{T}^{1}\left(\dot{B}_{N, \infty}^{-1}\right)$. La proposition 3.11 assure que

$$
\bar{u} \in L_{T}^{\infty}\left(\dot{B}_{N, \infty}^{-1}\right) \cap \widetilde{L}_{T}^{1}\left(\dot{B}_{N, \infty}^{1}\right) \quad \text { et } \quad \nabla \bar{\Pi} \in \widetilde{L}_{T}^{1}\left(\dot{B}_{N, \infty}^{-1}\right) .
$$

Cela achève la démonstration de la proposition.

La démonstration de l'existence globale dans le théorème 1.4 repose sur des estimations a priori adéquates qui font l'objet de la partie suivante.

5.2.2. Estimations a priori. - Considérons une solution régulière $(\theta, u, \nabla \Pi)$ du système de Boussinesq. Remarquons tout d'abord que la vitesse $u$ vérifie

$$
u(t)=e^{\nu t \Delta} u_{0}+\int_{0}^{t} e^{\nu(t-s) \Delta} \mathcal{P} \operatorname{div}(u \otimes u)(s) d s+\int_{0}^{t} e^{\nu(t-s) \Delta} \mathcal{P}\left(\theta(s) e_{N}\right) d s .
$$

Donc, en vertu des lemmes 4.1 et 4.2 , et sachant que $\|u \otimes u\|_{L^{\frac{N}{2}, \infty}} \leq\|u\|_{L^{N, \infty}}^{2}$, on a

$$
\|u\|_{L_{t}^{\infty}\left(L^{N, \infty}\right)} \leq C\left(\left\|u_{0}\right\|_{L^{N, \infty}}+\nu^{-1}\|u\|_{L_{t}^{\infty}\left(L^{N, \infty}\right)}^{2}+\nu^{-1}\|\theta\|_{L_{t}^{\infty}\left(L^{\frac{N}{3}, \infty}\right)}\right) .
$$

Comme par ailleurs (cf proposition 4.6),

$$
\|\theta\|_{L_{t}^{\infty}\left(L^{\frac{N}{3}, \infty}\right)}=\left\|\theta_{0}\right\|_{L^{\frac{N}{3}, \infty}}
$$

on en conclut que

$$
\|u\|_{L_{t}^{\infty}\left(L^{N, \infty}\right)} \leq c \nu
$$

pourvu que

$$
\nu^{-1}\left\|\theta_{0}\right\|_{L^{\frac{N}{3}, \infty}}+\left\|u_{0}\right\|_{L^{N, \infty}} \leq c^{\prime} \nu
$$

avec $c^{\prime}$ suffisamment petit. 
Il s'agit maintenant de propager la régularité nécessaire pour pouvoir établir l'existence globale et l'unicité. Tout d'abord, les lemmes 4.3 et 4.5 , et le fait que $L^{N, \infty} \hookrightarrow \dot{C}^{-1}$ (voir le lemme 3.9) assurent que

$$
U(t):=\|u\|_{L_{t}^{\infty}\left(\dot{B}_{p, 1}^{-1+\frac{N}{p}}\right)}+\nu\|u\|_{L_{t}^{1}\left(\dot{B}_{p, 1}^{1+\frac{N}{p}}\right)} \leq C\left(\left\|u_{0}\right\|_{\dot{B}_{p, 1}^{-1+\frac{N}{p}}}+\|\theta\|_{L_{t}^{1}\left(\dot{B}_{p, 1}^{-1+\frac{N}{p}}\right)}\right)
$$

pour tout $p \in[N, \infty]$ pourvu que (51) soit satisfaite. Pour cela, il suffit que les données initiales vérifient (52), ce que nous supposerons désormais.

D'autre part, en vertu de la proposition 4.8,

$$
\|\theta(t)\|_{\dot{B}_{N, 1}^{0}} \leq C\left\|\theta_{0}\right\|_{\dot{B}_{N, 1}^{0}}\left(1+\|u\|_{L_{t}^{1}\left(\dot{B}_{N, 1}^{2}\right)}\right) .
$$

Sachant que $\dot{B}_{N, 1}^{0} \hookrightarrow \dot{B}_{p, 1}^{-1+\frac{N}{p}}$, on obtient donc

$$
U(t) \leq C\left\|u_{0}\right\|_{\dot{B}_{p, 1}^{-1+\frac{N}{p}}}+C t\left\|\theta_{0}\right\|_{\dot{B}_{N, 1}^{0}}+C \nu^{-1}\left\|\theta_{0}\right\|_{\dot{B}_{N, 1}^{0}} \int_{0}^{t} U(\tau) d \tau,
$$

puis grâce au lemme de Gronwall,

$$
U(t) \leq C\left\|u_{0}\right\|_{\dot{B}_{p, 1}^{-1+\frac{N}{p}}} e^{C t \nu^{-1}\left\|\theta_{0}\right\|_{\dot{B}_{N, 1}^{0}}}+\nu\left(e^{C t \nu^{-1}\left\|\theta_{0}\right\|_{\dot{B}_{N, 1}^{0}}^{0}}-1\right) .
$$

5.2.3. Démonstration de l'existence globale dans le cas $p=N$. - Soit $T^{*}$ le temps d'existence de la solution $(\theta, u, \nabla \Pi)$ définie dans le théorème 1.3. Sachant que $\theta_{0} \in L^{\frac{N}{3}, \infty}$ et que $\theta$ est transporté par un champ de vecteurs qui est dans $L_{l o c}^{1}\left(0, T^{*} ; \operatorname{Lip}\right)$, on a $\theta \in L^{\infty}\left(0, T^{*} ; L^{\frac{N}{3}, \infty}\right)$ avec norme constante. Comme de plus $u_{0} \in L^{N, \infty}$, les lemmes 4.1 et 4.2 permettent d'affirmer que $u \in L_{l o c}^{\infty}\left(0, T^{*} ; L^{N, \infty}\right)$.

D'autre part, en utilisant le schéma itératif (40), on obtient une estimation uniforme dans $L^{\infty}\left(0, T^{*} ; L^{N, \infty}\right)$. En effet, on a pour tout $t \in\left[0, T^{*}[\right.$,

$$
\left\|u^{n+1}(t)\right\|_{L^{N, \infty}} \leq C\left(\left\|u_{0}\right\|_{L^{N, \infty}}+\nu^{-1}\left\|\theta_{0}\right\|_{L^{\frac{N}{3}, \infty}}+\nu^{-1}\left\|u^{n}\right\|_{L_{t}^{\infty}\left(L^{N, \infty}\right)}^{2}\right)
$$

ce qui implique par récurrence que $\left\|u^{n}\right\|_{L_{t}^{\infty}\left(L^{N, \infty}\right)}<2 C\left(\left\|u_{0}\right\|_{L^{N, \infty}}+\right.$ $\left.\nu^{-1}\left\|\theta_{0}\right\|_{L^{\frac{N}{3}, \infty}}\right)<2 C c \nu$ si (52) est satisfaite. Par passage à la limite on obtient $\|u\|_{L^{\infty}\left(0, T^{*} ; L^{N, \infty}\right)} \leq 2 C c \nu$.

Supposons par l'absurde que $T^{*}$ est fini. Alors les estimations a priori de la section 5.2.2 assurent que

$$
\theta \in L_{T^{*}}^{\infty}\left(\dot{B}_{N, 1}^{0}\right) \text { et } u \in L_{T^{*}}^{\infty}\left(\dot{B}_{N, 1}^{0}\right) \cap L_{T^{*}}^{1}\left(\dot{B}_{N, 1}^{2}\right) .
$$

Le théorème 1.3 permet alors de prolonger la solution au-delà de $T^{*}$, ce qui contredit la maximalité de $T^{*}$. Cela achève la démonstration du théorème 1.4 dans le cas $p=N$. 
5.2.4. Démonstration de l'existence globale dans le cas $p>N$. - La stratégie est des plus simples : régulariser et tronquer la vitesse initiale afin de se ramener au cas $p=N$ que l'on sait traiter, puis passer à la limite.

Pour la régularisation des données, nous ferons appel au lemme suivant :

Lemme 5.2. - Soit $\psi \in \mathcal{C}_{c}^{\infty}\left(\mathbb{R}^{N}\right)$ et $(s, p)$ tels que $1 \leq p \leq \infty$ et $-N / p^{\prime}<s \leq$ $N / p$. Pour $R>0$, notons $\psi_{R}=\psi(\dot{\bar{R}})$. Il existe une constante $C$ indépendante de $R$ telle que pour toute fonction $f$ de $\dot{B}_{p, 1}^{s}$, on ait

$$
\left\|\psi_{R} f\right\|_{\dot{B}_{p, 1}^{s}} \leq C\|f\|_{\dot{B}_{p, 1}^{s}} .
$$

Preuve. - Vu les hypothèses sur $s$ et $p$, la proposition 3.14 donne

$$
\left\|\psi_{R} f\right\|_{\dot{B}_{p, 1}^{s}} \leq C\left(\left\|\psi_{R}\right\|_{\dot{B}_{p, 1}^{\frac{N}{p}}}+\left\|\psi_{R}\right\|_{\dot{B}_{p^{\prime}, 1}^{\frac{N}{p^{\prime}}}}\right)\|f\|_{\dot{B}_{p, 1}^{s}} .
$$

Les normes de $\psi_{R}$ considérées sont bien finies car $\mathcal{C}_{c}^{\infty}$ est un sous-espace de $\dot{B}_{r, 1}^{\frac{N}{r}}$ pour tout $r \in[1,+\infty]$. Par ailleurs, les normes dans ces espaces sont invariantes par changement d'échelle, et ne dépendent donc pas de $R$.

5.2.5. Régularisation des données et résolution. - On pose

$$
u_{0}^{n}=\psi\left(n^{-1} \cdot\right) \sum_{|q| \leq n} \dot{\Delta}_{q} u_{0}
$$

où $\psi$ est une fonction de $\mathcal{C}_{c}^{\infty}(B(0,2))$ à valeurs dans $[0,1]$ et égale à 1 sur $B(0,1)$. Il est clair que $u_{0}^{n}$ converge vers $u_{0}$ au sens des distributions. De plus, d'après le lemme ci-dessus, il existe une constante $C$ telle que

$$
\forall n \in \mathbb{N},\left\|u_{0}^{n}\right\|_{\dot{B}_{p, 1}^{\frac{N}{p}-1}} \leq C\left\|u_{0}\right\|_{\dot{B}_{p, 1}^{\frac{N}{p}-1}}
$$

et l'on vérifie facilement en utilisant des inégalités de convolution que

$$
\forall n \in \mathbb{N},\left\|u_{0}^{n}\right\|_{L^{N, \infty}} \leq C\left\|u_{0}\right\|_{L^{N, \infty}} .
$$

En appliquant le théorème 1.4 avec $p=N$, on constate que si $\nu^{-2}\left\|\theta_{0}\right\|_{L^{\frac{N}{3}, \infty}}+$ $\nu^{-1}\left\|u_{0}\right\|_{L^{N, \infty}}$ est suffisamment petit alors on peut alors résoudre globalement le système de Boussinesq avec données $\left(\theta_{0}^{n}, u_{0}^{n}\right)$. La solution $\left(\theta^{n}, u^{n}, \nabla \Pi^{n}\right)$ ainsi obtenue appartient à

$$
\mathcal{C}\left(\mathbb{R}_{+} ; \dot{B}_{N, 1}^{0}\right) \times\left(\mathcal{C}\left(\mathbb{R}_{+} ; \dot{B}_{N, 1}^{0}\right) \cap L_{l o c}^{1}\left(\mathbb{R}_{+} ; \dot{B}_{N, 1}^{2}\right)^{N} \times\left(L_{l o c}^{1}\left(\mathbb{R}_{+} ; \dot{B}_{N, 1}^{0}\right)^{N}\right.\right.
$$

5.2.6. Estimations a priori globales. - Par injection dans les espaces de Besov, $\left(\theta^{n}, u^{n}, \nabla \Pi^{n}\right)$ appartient aussi à l'espace $E^{p}:=\mathcal{C}\left(\mathbb{R}_{+} ; \dot{B}_{N, 1}^{0}\right) \times\left(\mathcal{C}\left(\mathbb{R}_{+} ; \dot{B}_{p, 1}^{\frac{N}{p}-1}\right) \cap L_{l o c}^{1}\left(\mathbb{R}_{+} ; \dot{B}_{p, 1}^{\frac{N}{p}+1}\right)^{N} \times\left(L_{l o c}^{1}\left(\mathbb{R}_{+} ; \dot{B}_{p, 1}^{\frac{N}{p}-1}\right)^{N}\right.\right.$ Ainsi les estimations a priori de la partie 5.2.2 s'appliquent telles quelles. On en déduit des bornes uniformes sur $\left(\theta^{n}, u^{n}, \nabla \Pi^{n}\right)$ dans l'espace $E^{p}$. 
5.2.7. Convergence. - La convergence de $\left(\theta^{n}, u^{n}, \nabla \Pi^{n}\right)_{n \in \mathbb{N}}$ repose sur des arguments de compacité qui peuvent être déduits des propriétés des dérivées temporelles.

Fixons un temps $T>0$. Par construction, on a

$$
\partial_{t} \theta^{n}=-\operatorname{div}\left(u^{n} \theta^{n}\right) .
$$

Sachant que la suite $\left(u^{n}\right)_{n \in \mathbb{N}}$ est bornée dans $L_{T}^{2}\left(\dot{B}_{p, 1}^{\frac{N}{p}}\right) \hookrightarrow L_{T}^{2}\left(L^{\infty}\right)$ et que la suite $\left(\theta^{n}\right)_{n \in \mathbb{N}}$ est bornée dans $L_{T}^{\infty}\left(\dot{B}_{N, 1}^{0}\right) \hookrightarrow L_{T}^{\infty}\left(L^{N}\right)$, on en déduit que $\left(\partial_{t} \theta^{n}\right)_{n \in \mathbb{N}}$ est bornée dans $L_{T}^{2}\left(W^{-1, N}\right)$. Cela permet d'affirmer que $\left(\theta^{n}\right)_{n \in \mathbb{N}}$ est bornée dans $\mathcal{C}\left([0, T] ; L^{N}\right)$ et équicontinue de $[0, T]$ dans $W^{-1, N}$. Sachant que l'injection de $L^{N}$ dans $W^{-1, N}$ est localement compacte, on peut en déduire en combinant théorème d'Ascoli et procédé d'extraction diagonal la convergence d'une sous-suite de $\left(\theta^{n}\right)_{n \in \mathbb{N}}$ vers une distribution $\theta$ qui appartient localement à $\mathcal{C}\left([0, T] ; W^{-1, N}\right)$.

De même, un examen attentif de l'expression de $\left(\partial_{t} u^{n}\right)_{n \in \mathbb{N}}$ permet d'affirmer que $\left(u^{n}\right)_{n \in \mathbb{N}}$ est équicontinue de $[0, T]$ dans $B_{N, \infty}^{-2}$ puis de conclure à la convergence à extraction près. Les détails sont laissés au lecteur.

5.2.8. Conclusion. - Tout d'abord, les bornes uniformes sur $\left(\theta^{n}, u^{n}\right)$ permettent d'affirmer que $(\theta, u)$ appartient en fait à

$$
L_{l o c}^{\infty}\left(\mathbb{R}_{+} ; \dot{B}_{N, 1}^{0} \cap L^{\frac{N}{3}, \infty}\right) \times\left(L_{l o c}^{\infty}\left(\mathbb{R}_{+} ; \dot{B}_{p, 1}^{\frac{N}{p}-1} \cap L^{N, \infty}\right) \cap L_{l o c}^{1}\left(\mathbb{R}_{+} ; \dot{B}_{p, 1}^{\frac{N}{p}+1}\right)\right)^{N}
$$

Notons que, même dans le cas $N=3$ où il faut remplacer $L^{\frac{N}{3}, \infty}$ par $L^{1}$, les bornes uniformes dans l'espace de Besov $\dot{B}_{N, 1}^{0}$ (qui s'injecte continûment dans $L^{N}$ ) empêchent d'éventuelles concentrations pour $\theta$.

Par interpolation entre les bornes uniformes et les résultats de convergence, on peut montrer que la suite $\left(\theta^{n}, u^{n}\right)_{n \in \mathbb{N}}$ converge en un sens suffisamment fort pour pouvoir passer à la limite dans tous les termes de l'équation.

Enfin, sachant que $(\theta, u)$ est solution, il est facile de montrer que $\partial_{t} \theta+$ $u \cdot \nabla \theta \in L_{l o c}^{1}\left(\mathbb{R}_{+} ; \dot{B}_{N, 1}^{0}\right)$ et que $\partial_{t} u-\nu \Delta u \in L_{l o c}^{1}\left(\mathbb{R}_{+} ; \dot{B}_{p, 1}^{\frac{N}{p}-1}\right)$, ce qui assure l'appartenance de $(\theta, u)$ à $\mathcal{C}\left(\mathbb{R}_{+} ; \dot{B}_{N, 1}^{0}\right) \times \mathcal{C}\left(\mathbb{R}_{+} ; \dot{B}_{p, 1}^{\frac{N}{p}-1}\right)$.

\section{Unicité des solutions d'énergie finie en dimension deux}

Cette section est consacrée à la démonstration du théorème 1.2. Rappelons que pour n'importe quelle donnée $\left(\theta_{0}, u_{0}\right)$ dans $L^{2}\left(\mathbb{R}^{2}\right)$ (avec div $u_{0}=0$ ), le théorème 1.1 assure l'existence globale d'au moins une solutions faible d'énergie finie pour le système de Boussinesq. Nous nous proposons de démontrer l'unicité d'une telle solution. 
Dans un premier temps, on va établir que le champ de vitesse appartient nécessairement à l'espace $\widetilde{L}_{l o c}^{1}\left(\mathbb{R}_{+} ; H^{2}\left(\mathbb{R}^{2}\right)\right)$. Cette propriété remarquable (qui nous permettra d'utiliser les estimations avec perte de régularité énoncées dans la proposition 4.9 afin d'établir l'unicité) a été établie par J.-Y. Chemin et N. Lerner dans [10] pour le système de Navier-Stokes standard. L'objet du lemme ci-dessous est de démontrer que ce résultat demeure pour le système de Boussinesq en dimension deux.

LEMme 6.1. - Soit $(\theta, u, \nabla \Pi)$ une solution de (1) sur $\mathbb{R}_{+} \times \mathbb{R}^{2}$ appartenant à l'espace d'énergie :

$$
\theta \in L^{\infty}\left(\mathbb{R}_{+} ; L^{2}\left(\mathbb{R}^{2}\right)\right) \quad \text { et } \quad u \in L_{\text {loc }}^{\infty}\left(\mathbb{R}_{+} ; L^{2}\left(\mathbb{R}^{2}\right)\right) \cap L^{2}\left(\mathbb{R}_{+} ; H^{1}\left(\mathbb{R}^{2}\right)\right),
$$

Alors on a $u \in \widetilde{L}_{\text {loc }}^{1}\left(\mathbb{R}_{+} ; H^{2}\left(\mathbb{R}^{2}\right)\right) \cap \widetilde{\mathcal{C}}_{\text {loc }}\left(\mathbb{R}_{+} ; L^{2}\right)$.

Démonstration. - En appliquant l'opérateur de projection $\mathcal{P}$ à l'équation de la vitesse, on constate que $u$ vérifie l'équation de la chaleur :

$$
\left\{\begin{array}{l}
\partial_{t} u-\nu \Delta u=f_{1}+f_{2} \\
\left.u\right|_{t=0}=u_{0} \in L^{2}\left(\mathbb{R}^{2}\right)
\end{array} \quad \text { avec } \quad f_{1}=-\mathcal{P} \operatorname{div}(u \otimes u) \quad \text { et } \quad f_{2}=\mathcal{P}\left(\theta e_{2}\right)\right.
$$

Cela nous amène à décomposer $u$ en $u_{1}+u_{2}$ avec

$$
u_{1}=\int_{0}^{t} e^{\nu(t-\tau) \Delta} f_{1}(\tau) d \tau \quad \text { et } \quad u_{2}=e^{t \nu \Delta} u_{0}+\int_{0}^{t} e^{\nu(t-\tau) \Delta} f_{2}(\tau) d \tau .
$$

Il est clair que $f_{2} \in L_{l o c}^{1}\left(\mathbb{R}_{+} ; L^{2}\right)$. De plus, par interpolation, on a visiblement $u \in L_{l o c}^{\frac{8}{3}}\left(\mathbb{R}_{+} ; H^{\frac{3}{4}}\right)$. En utilisant les lois de produit dans les espaces de Besov non homogènes (qui sont identiques à celles que nous avons énoncées sous la proposition 3.14 pour les espaces homogènes), on en déduit que $u \otimes u$ appartient à $L_{\text {loc }}^{\frac{4}{3}}\left(\mathbb{R}_{+} ; B_{2,1}^{\frac{1}{2}}\right)$ puis que $f_{1} \in L_{\text {loc }}^{\frac{4}{3}}\left(\mathbb{R}_{+} ; B_{2,1}^{-\frac{1}{2}}\right)$.

Finalement, la proposition 3.11 et la relation (19) garantissent donc que

$$
u_{1} \in L_{l o c}^{\frac{4}{3}}\left(\mathbb{R}_{+} ; B_{2,1}^{\frac{3}{2}}\right) \cap \mathcal{C}\left(\mathbb{R}_{+} ; B_{2,1}^{0}\right) \quad \text { et } \quad u_{2} \in \widetilde{L}_{l o c}^{1}\left(\mathbb{R}_{+} ; H^{2}\right) \cap \widetilde{\mathcal{C}}_{l o c}\left(\mathbb{R}_{+} ; L^{2}\right)
$$

On découpe alors $f_{1}$ en trois termes :

$$
f_{1}=\mathcal{P} \operatorname{div}\left(u_{1} \otimes u_{1}\right)+\mathcal{P}\left(u_{1} \cdot \nabla u_{2}\right)+\mathcal{P}\left(u_{2} \cdot \nabla u\right) .
$$

D'après (55) et par interpolation, on a $u_{1} \in L_{\text {loc }}^{2}\left(\mathbb{R}_{+} ; B_{2,1}^{1}\right)$. Sachant que $B_{2,1}^{1}\left(\mathbb{R}^{2}\right)$ est une algèbre, on en déduit que

$$
\operatorname{div}\left(u_{1} \otimes u_{1}\right) \in L_{l o c}^{1}\left(\mathbb{R}_{+} ; B_{2,1}^{0}\right) \hookrightarrow L_{l o c}^{1}\left(\mathbb{R}_{+} ; L^{2}\right) .
$$

Par ailleurs, en utilisant le fait que $B_{2,1}^{1}\left(\mathbb{R}^{2}\right) \hookrightarrow L^{\infty}\left(\mathbb{R}^{2}\right)$ et que $\nabla u_{2} \in$ $L_{\text {loc }}^{2}\left(\mathbb{R}_{+} ; L^{2}\right)$, on établit aisément que

$$
u_{1} \cdot \nabla u_{2} \in L_{l o c}^{1}\left(\mathbb{R}_{+} ; L^{2}\right) .
$$

TOME $136-2008-\mathrm{N}^{\circ} 2$ 
Enfin, en combinant le fait que $u_{2} \in \widetilde{L}_{\text {loc }}^{4}\left(\mathbb{R}_{+} ; H^{\frac{1}{2}}\right)$ et que $\nabla u \in \widetilde{L}_{l o c}^{\frac{4}{3}}\left(\mathbb{R}_{+} ; H^{\frac{1}{2}}\right)$, on trouve que

$$
u_{2} \cdot \nabla u \in L_{l o c}^{1}\left(\mathbb{R}_{+} ; L^{2}\right) .
$$

Comme l'opérateur $\mathcal{P}$ est continu sur $L^{2}$, on peut conclure que $f_{1} \in$ $L_{l o c}^{1}\left(\mathbb{R}_{+} ; L^{2}\right)$ puis, grâce à la proposition 3.11 , que $u_{1} \in \widetilde{L}_{\text {loc }}^{1}\left(\mathbb{R}_{+} ; H^{2}\right) \cap$ $\widetilde{\mathcal{C}}_{\text {loc }}\left(\mathbb{R}_{+} ; L^{2}\right)$.

REMARque 6.2. - En étant plus soigneux, on peut établir que $f_{1} \in$ $L_{\text {loc }}^{1}\left(\mathbb{R}_{+} ; B_{2,1}^{0}\right)$, ce qui entraîne que

$$
u_{1} \in \widetilde{\mathcal{C}}_{l o c}\left(\mathbb{R}_{+} ; \dot{B}_{2,1}^{0}\right) \cap L_{l o c}^{1}\left(\mathbb{R}_{+} ; \dot{B}_{2,1}^{2}\right) .
$$

On retrouve ainsi le fait (bien connu pour les équations de Navier-Stokes) que $l a$ fluctuation est plus régulière que la solution libre du système de Stokes. Cependant cette petite amélioration ne sera pas nécessaire pour démontrer l'unicité dans le théorème 1.2.

Nous sommes maintenant armés pour démontrer l'unicité. Considérons donc deux solutions $\left(\theta_{1}, u_{1}, \nabla \Pi_{1}\right)$ et $\left(\theta_{2}, u_{2}, \nabla \Pi_{2}\right)$ de (1) avec même donnée initiale $\left(\theta_{0}, u_{0}\right)$ dans $L^{2}\left(\mathbb{R}^{2}\right)$. Le système vérifié par la différence $(\delta \theta, \delta u, \nabla \delta \Pi)$ entre ces solutions est

$$
\left\{\begin{array}{l}
\partial_{t} \delta \theta+\operatorname{div}\left(u_{1} \delta \theta\right)=-\operatorname{div}\left(\theta_{2} \delta u\right) \\
\partial_{t} \delta u+\operatorname{div}\left(u_{1} \otimes \delta u\right)-\nu \Delta \delta u+\nabla \delta \Pi=-\operatorname{div}\left(\delta u \otimes u_{2}\right)+\delta \theta e_{2} .
\end{array}\right.
$$

Le caractère hyperbolique de la première équation induit une perte d'une dérivée dans les estimations de stabilité. Il va donc falloir démontrer des estimations au niveau $H^{-1}$ au lieu de $L^{2}$. Pour une raison purement technique, et comme l'on dispose d'une grande marge de manœuvre, il est plus commode d'opérer au niveau $H^{-3 / 2}$ (mais n'importe quel exposant strictement compris entre -2 et -1 conviendrait aussi).

Pour estimer $\delta \theta$ et $\delta u$, nous allons donc appliquer la proposition 4.9 (ou plutôt la remarque 4.10 pour la deuxième équation) avec $s=-3 / 2$ aux équations du système. Pour que cela soit possible, fixons un temps $T>0$ tel que la condition (37) de la proposition 4.9 soit satisfaite par le champ de vitesses $u_{1}$. Le lemme 6.1 garantit que $u_{1} \in \widetilde{L}_{T}^{1}\left(H^{2}\right)$ et donc l'existence d'un tel $T$. En notant

$$
\begin{aligned}
& \varepsilon_{q}(t)=C \sum_{q^{\prime} \leq q} 2^{2 q^{\prime}}\left\|\check{\Delta}_{q^{\prime}} u_{1}\right\|_{L_{t}^{1}\left(L^{2}\right)} \quad \delta \Theta(t):=\sup _{\substack{\tau \in[0, t] \\
q \geq-1}} 2^{-\frac{3}{2} q-\varepsilon_{q}(\tau)}\left\|\Delta_{q} \delta \theta(\tau)\right\|_{L^{2}}, \\
& \delta U(t):=\sup _{\substack{\tau \in[0, t] \\
q \geq-1}} 2^{-\frac{3}{2} q-\varepsilon_{q}(\tau)}\left\|\Delta_{q} \delta u\right\|_{L^{2}}+\nu \sup _{q \geq-1} \int_{0}^{t} 2^{\frac{q}{2}-\varepsilon_{q}(\tau)}\left\|\Delta_{q} \delta u\right\|_{L^{2}} d \tau,
\end{aligned}
$$


on obtient alors

$$
\begin{aligned}
& \delta \Theta(t) \leq C \sup _{q \geq-1} \int_{0}^{t} 2^{-\frac{3}{2} q-\varepsilon_{q}(\tau)}\left\|\Delta_{q} \operatorname{div}\left(\theta_{2} \delta u\right)\right\|_{L^{2}} d \tau \\
& \delta U(t) \leq C(1+\nu t) \sup _{q \geq-1} \int_{0}^{t} 2^{-\frac{3}{2} q-\varepsilon_{q}(\tau)}\left(\left\|\Delta_{q} \operatorname{div}\left(\delta u \otimes u_{2}\right)\right\|_{L^{2}}+\left\|\Delta_{q} \delta \theta\right\|_{L^{2}}\right) d \tau .
\end{aligned}
$$

L'inoffensif facteur $(1+\nu t)$ provient du fait que l'on a pris le sup pour $q \geq-1$ et non pas seulement pour $q \geq 0$ dans le second terme de $\delta U(t)$.

Admettons que les termes de convection se majorent comme suit (voir la démonstration en appendice) :

$$
\begin{aligned}
& \sup _{q \geq-1} \int_{0}^{t} 2^{-\frac{3}{2} q-\varepsilon_{q}(\tau)}\left\|\Delta_{q} \operatorname{div}\left(\theta_{2} \delta u\right)\right\|_{L^{2}} d \tau \\
& \leq C\left\|\theta_{2}\right\|_{L_{t}^{\infty}\left(B_{2, \infty}^{0}\right)} \sup _{q} \int_{0}^{t} 2^{\frac{q}{2}-\varepsilon_{q}(\tau)}\left\|\Delta_{q} \delta u\right\|_{L^{2}} d \tau \\
& \sup _{q \geq-1} \int_{0}^{t} 2^{-\frac{3}{2} q-\varepsilon_{q}(\tau)}\left\|\Delta_{q} \operatorname{div}\left(\delta u \otimes u_{2}\right)\right\|_{L^{2}} d \tau \\
& \leq C\left\|u_{2}\right\|_{\widetilde{L}_{t}^{2}\left(B_{2, \infty}^{1}\right)} \sup _{q \geq-1}\left\|2^{-\frac{q}{2}-\varepsilon_{q}(\tau)}\right\| \Delta_{q} \delta u\left\|_{L^{2}}\right\|_{L_{t}^{2}} .
\end{aligned}
$$

En remarquant que pour tout $q \geq-1$, on a

$$
\left\|2^{-\frac{q}{2}-\varepsilon_{q}(\tau)}\right\| \Delta_{q} \delta u\left\|_{L^{2}}\right\|_{L_{t}^{2}}^{2} \leq\left\|2^{\frac{q}{2}-\varepsilon_{q}(\tau)}\right\| \Delta_{q} \delta u\left\|_{L^{2}}\right\|_{L_{t}^{1}}\left\|2^{-\frac{3}{2} q-\varepsilon_{q}(\tau)}\right\| \Delta_{q} \delta u\left\|_{L^{2}}\right\|_{L_{t}^{\infty}},
$$

on obtient donc

$$
\begin{aligned}
& \delta \Theta(t) \leq C \nu^{-1}\left\|\theta_{2}\right\|_{L_{t}^{\infty}\left(B_{2, \infty}^{0}\right)} \delta U(t), \\
& \delta U(t) \leq C(1+\nu t)\left(\nu^{-\frac{1}{2}}\left\|u_{2}\right\|_{\widetilde{L}_{t}^{2}\left(B_{2, \infty}^{1}\right)} \delta U(t)+\int_{0}^{t} \delta \Theta(\tau) d \tau\right) .
\end{aligned}
$$

Sachant que $\left\|u_{2}\right\|_{\widetilde{L}_{t}^{2}\left(B_{2, \infty}^{1}\right)} \leq C\left\|u_{2}\right\|_{L_{t}^{2}\left(H^{1}\right)}$ et que $\left\|\theta_{2}\right\|_{L_{t}^{\infty}\left(B_{2, \infty}^{0}\right)} \leq C\left\|\theta_{2}\right\|_{L_{t}^{\infty}\left(L^{2}\right)} \leq$ $C\left\|\theta_{0}\right\|_{L^{2}}$, on en déduit que pour $t$ assez petit, on a

$$
\delta U(t) \leq C(1+\nu t) \int_{0}^{t} \delta \Theta(\tau) d \tau \quad \text { et } \quad \delta \Theta(t) \leq C \nu^{-1}\left\|\theta_{0}\right\|_{L^{2}} \delta U(t) .
$$

Le lemme de Gronwall permet alors de conclure à l'unicité sur un petit intervalle de temps.

L'unicité globale s'obtient par un argument de continuité en temps et de connexité des plus classiques. 
Fin de la démonstration du théorème 1.2. - Pour montrer que l'égalité d'énergie (3) est satisfaite, il suffit de remarquer que $u$ vérifie le système de Navier-Stokes incompressible en dimension deux avec terme de force dans $L^{\infty}\left(\mathbb{R}^{+} ; L^{2}\right)$. Enfin, la théorie classique des équations de transport assure que la norme $L^{2}$ de $\theta$ est conservée au cours de l'évolution. Un argument standard d'analyse fonctionnelle permet par ailleurs de montrer que $\theta$ est faiblement continue en temps à valeurs $L^{2}$. Comme la norme $L^{2}$ est conservée, on en déduit que la continuité est forte. Cela achève la démonstration du théorème 1.2.

\section{Quelques résultats supplémentaires}

7.1. Un critère de prolongement. — On peut démontrer un critère de prolongement bien plus précis que celui qui est énoncé à la fin du théorème 1.3. En fait, si

$$
\int_{0}^{T^{*}}\|\nabla u(\tau)\|_{L^{\infty}} d \tau<\infty
$$

alors la solution peut être prolongée au-delà du temps $T^{*}$.

Cela résulte du fait que l'intégrand apparaissant dans l'inégalité de la proposition 4.7 peut être remplacé par $\|\nabla u\|_{L^{\infty}}$ lorsque $|s|<1$. Il en est donc de même dans la proposition 4.8. En utilisant le théorème 1 de [15], on peut alors contrôler la norme $L^{\infty}\left(0, T^{*} ; \dot{B}_{N, 1}^{0}\right)$ de la solution par $\int_{0}^{T^{*}}\|\nabla u(\tau)\|_{L^{\infty}} d \tau$. Les détails sont laissés au lecteur.

7.2. Conditions aux limites périodiques. - Rappelons qu'il existe une version périodique de la décomposition de Littlewood-Paley. De ce fait, tous nos résultats sont encore valables mutatis mutandis dans le tore $\mathbb{T}^{N}$. En fait, sachant que toute solution périodique à moyenne nulle du système de Stokes décroît exponentiellement en temps, le cas périodique est même plus facile à traiter.

7.3. Termes de forces. - Notons tout d'abord que nos méthodes n'utilisent jamais la verticalité du terme $\theta e_{N}$. Nos résultats peuvent donc s'adapter au cas plus général où $\theta$ est à valeurs dans $\mathbb{R}^{N}$.

Par ailleurs, pour simplifier la présentation, nous avons omis les termes de forces extérieures dans le système de Boussinesq. Indiquons rapidement les hypothèses raisonnables à imposer sur ces termes.

Tout d'abord, si l'on note $\Theta$ le terme de source pour l'équation sur la température, et $f$ le terme de forces extérieures pour l'équation sur la vitesse, on peut obtenir un analogue du théorème 1.1 si l'on suppose en sus que

$$
\Theta \in L_{l o c}^{1}\left(\mathbb{R}^{+} ; L^{p}\right) \quad \text { et } \quad f \in L_{l o c}^{1}\left(\mathbb{R}^{+} ; L^{2}\right) \cup L_{l o c}^{2}\left(\mathbb{R}^{+} ; \dot{H}^{-1}\right) .
$$


Pour démontrer l'unicité en dimension deux, il est cependant impératif que $\Theta$ et $f$ soient dans $L_{l o c}^{1}\left(\mathbb{R}^{+} ; L^{2}\right)$ afin que le champ de vitesses appartienne à $\widetilde{L}_{\text {loc }}^{1}\left(\mathbb{R}^{+} ; H^{2}\right)$.

En ce qui concerne les solutions fortes globales, nos résultats globaux demeurent si l'on suppose que :

- $\Theta$ appartient à $L_{l o c}^{1}\left(\mathbb{R}^{+} ; \dot{B}_{N, 1}^{0}\right)$ et est petite dans $L^{1}\left(\mathbb{R}^{+} ; L^{\frac{N}{3}, \infty}\right)$ (resp. $L^{1}\left(\mathbb{R}^{+} ; L^{1}\right)$ si $\left.N=3\right)$,

- $f$ est dans $L_{\text {loc }}^{1}\left(\mathbb{R}^{+} ; \dot{B}_{p, 1}^{-1+\frac{N}{p}}\right)$ et est petite dans $L^{p}\left(\mathbb{R}^{+} ; L^{\frac{N(p+2)}{3 p}, \infty}\right)$ pour un $p \in[1, \infty]\left(\right.$ resp. $L^{1}\left(\mathbb{R}^{+} ; L^{1}\right)$ si $N=3$ et $\left.p=1\right)$.

7.4. Modèle complètement visqueux. - Tous nos résultats demeurent valables sans aucune hypothèse supplémentaire pour le système de Boussinesq complètement visqueux :

$$
\left\{\begin{array}{l}
\partial_{t} \theta+u \cdot \nabla \theta-\kappa \Delta \theta=0, \\
\partial_{t} u+u \cdot \nabla u-\nu \Delta u+\nabla \Pi=\theta e_{N}, \\
\operatorname{div} u=0,
\end{array}\right.
$$

avec $\kappa$ paramètre positif (représentant la conductivité thermique si $\theta$ est une température).

Notre approche permet d'obtenir des estimations indépendantes du paramètre $\kappa$ sur les solutions construites et de démontrer ainsi des résultats de convergence pour $\kappa$ tendant vers 0 .

7.5. Non existence des singularités de type jet en dimension trois. - Pour le système de Navier-Stokes classique, il est bien connu que les solutions faibles d'énergie finie en dimension trois appartiennent à $L_{l o c}^{1}\left(\mathbb{R}_{+} ; L^{\infty}\right)$.

Pour le système de Boussinesq cette propriété est également connue pour des données dans $L^{2}\left(\mathbb{R}^{3}\right) \times H^{1}\left(\mathbb{R}^{3}\right)^{3}$, ce qui entraîne que les singularités de type jet (ou squirt en anglais) ne peuvent pas se produire (voir [11]).

Pour les équations de Navier-Stokes classiques avec données $L^{2}$, on peut établir une propriété bien plus forte, à savoir que le champ de vitesses est dans $L_{l o c}^{1}\left(\mathbb{R}_{+} ; \dot{B}_{2,1}^{\frac{3}{2}}\right)$ ce qui assure notamment l'existence d'un flot continu en temps et en espace (voir [9]). Cette propriété persiste pour le système de Boussinesq si $3 / 2<p \leq 2$ :

Proposition 7.1. - En dimension $N=3$, le champ de vitesses de toute solution faible d'énergie finie du système de Boussinesq avec données $\left(\theta_{0}, u_{0}\right) \in$ $L^{p}\left(\mathbb{R}^{3}\right) \times\left(L^{2}\left(\mathbb{R}^{3}\right)\right)^{3}($ pour $3 / 2<p \leq 2)$ appartient à $L_{\text {loc }}^{1}\left(\mathbb{R}_{+} ; B_{2,1}^{\frac{3}{2}}\right)$. 
Démonstration. - Nous présentons une démonstration différente de celle de [9]. Tout d'abord, comme, par construction, le champ de vitesses $u$ appartient à $L_{l o c}^{2}\left(\mathbb{R}_{+} ; H^{1}\right)$, les inclusions de Sobolev dans les espaces de Lorentz assurent que $u \in L_{\text {loc }}^{2}\left(\mathbb{R}_{+} ; L^{6,2}\right)$. En utilisant le fait que $\nabla u \in L_{\text {loc }}^{2}\left(\mathbb{R}_{+} ; L^{2}\right)$ et que $L^{2}$ coïncide avec l'espace de Lorentz $L^{2,2}$, on a donc $u \cdot \nabla u \in L_{l o c}^{1}\left(\mathbb{R}_{+} ; L^{3 / 2,1}\right)$. Mais par ailleurs, on a

$$
L^{\frac{3}{2}, 1}=\left(L^{1}, L^{2}\right)_{\frac{2}{3}, 1} \hookrightarrow\left(\dot{B}_{2, \infty}^{-\frac{3}{2}}, \dot{B}_{2, \infty}^{0}\right)_{\frac{2}{3}, 1}=\dot{B}_{2,1}^{-\frac{1}{2}},
$$

donc $\mathcal{P}(u \cdot \nabla u) \in L_{\text {loc }}^{1}\left(\mathbb{R}_{+} ; \dot{B}_{2,1}^{-1 / 2}\right)$. En observant que pour $3 / 2<p \leq 2$, on a $L^{p} \hookrightarrow B_{2,1}^{-\frac{1}{2}}$, on constate que $\mathcal{P}\left(\theta e_{3}\right) \in L_{\text {loc }}^{1}\left(\mathbb{R}_{+} ; B_{2,1}^{-1 / 2}\right)$. En vertu de la remarque 3.13 , on conclut alors que

$$
\int_{0}^{t} e^{\nu(t-s) \Delta} \mathcal{P}\left(\theta e_{3}-u \cdot \nabla u\right) d s \in L_{l o c}^{1}\left(\mathbb{R}_{+} ; B_{2,1}^{\frac{3}{2}}\right) .
$$

Enfin, sachant que $u_{0} \in L^{2}$, on a $e^{t \Delta} u_{0} \in L_{l o c}^{1}\left(\mathbb{R}_{+} ; H^{2-\varepsilon}\right)$ pour tout $\varepsilon>0$. Cela achève la démonstration du résultat.

\section{Appendice}

Nous démontrons ici le lemme qui nous a permis d'estimer les termes de convection dans la preuve de l'unicité du théorème 1.2.

Lemme 8.1. - Soit $\left(\alpha_{q}\right)_{q \geq-1}$ une suite de fonctions positives croissantes sur $[0, T]$, et $\varepsilon \in] 0,1\left[\right.$. On suppose que pour tout $q^{\prime} \geq q \geq-1$ et $t \in[0, T]$, on a

$$
0 \leq \alpha_{q^{\prime}}(t)-\alpha_{q}(t) \leq\left(\frac{1-\varepsilon}{2}\right)\left(q^{\prime}-q\right) .
$$

Alors pour tout $r \in] 1,+\infty]$, il existe une constante $C$ ne dépendant que de $r$ et de $\varepsilon$ telle que l'on ait l'estimation a priori suivante pour toute fonction a et champ de vecteurs $b$ à divergence nulle sur $\mathbb{R}^{2}$ :

$$
\begin{aligned}
& \sup _{q \geq-1} \int_{0}^{t} 2^{-q(1+\varepsilon)-\alpha_{q}(\tau)}\left\|\Delta_{q} \operatorname{div}(a b)\right\|_{L^{2}} d \tau \\
& \leq C\|b\|_{\widetilde{L}_{t}^{r}\left(B_{2, \infty}^{\frac{2}{r}}\right)} \sup _{q \geq-1}\left\|2^{q\left(1-\frac{2}{r}-\varepsilon\right)-\alpha_{q}}\right\| \Delta_{q} a\left\|_{L^{2}}\right\|_{L_{t}^{r^{\prime}}} .
\end{aligned}
$$

Démonstration. - La démonstration repose sur la décomposition de Bony, version non homogène. En tenant compte de $\operatorname{div} b=0$, on a (avec la convention habituelle de sommation sur les indices répétés) :

$$
\Delta_{q} \operatorname{div}(a b)=\Delta_{q}\left(T_{\partial_{j} b} a^{j}\right)+\Delta_{q}\left(T_{a^{j}} \partial_{j} b\right)+\Delta_{q} \partial_{j} R\left(a^{j}, b\right) .
$$


En vertu des propriétés de localisation de la décomposition de Littlewood-Paley, on a

$$
\Delta_{q}\left(T_{\partial_{j} b} a^{j}\right)=\sum_{\left|q^{\prime}-q\right| \leq 4} \Delta_{q}\left(S_{q^{\prime}-1} \partial_{j} b \Delta_{q^{\prime}} a^{j}\right) .
$$

Ci dessus les opérateurs $T$ et $R$ de paraproduit et de reste non homogènes se définissent comme $\dot{T}$ et $\dot{R}$ en remplaçant chaque bloc homogène $\dot{\Delta}_{q}$ par son homologue non homogène $\Delta_{q}$.

Pour simplifier les calculs, procédons comme si l'on avait $\Delta_{q}\left(T_{\partial_{j} b} a^{j}\right)=$ $S_{q-1} \partial_{j} b \Delta_{q} a^{j}$ (le fait d'avoir (56) permet de justifier cette approximation). En utilisant la définition de $S_{q-1}$, on obtient

$$
\left\|S_{q-1} \partial_{j} b \Delta_{q} a^{j}\right\|_{L^{2}} \leq 2^{q\left(2-\frac{2}{r}\right)}\left\|\Delta_{q} a\right\|_{L^{2}} \sum_{q^{\prime} \leq q-2} 2^{q^{\prime}\left(\frac{2}{r}-2\right)}\left\|\Delta_{q^{\prime}} \nabla b\right\|_{L^{\infty}} 2^{\left(q-q^{\prime}\right)\left(\frac{2}{r}-2\right)} .
$$

En conséquence, pour tout $0 \leq t \leq T$, on a

$$
\begin{aligned}
& \int_{0}^{t} 2^{-q(1+\varepsilon)-\alpha_{q}(\tau)}\left\|S_{q-1} \partial_{j} b \Delta_{q} a^{j}\right\|_{L^{2}} d \tau \\
& \quad \leq\left\|2^{q\left(1-\frac{2}{r}-\varepsilon\right)-\alpha_{q}}\right\| \Delta_{q} a\left\|_{L^{2}}\right\|_{L_{t}^{r^{\prime}}} \sum_{q^{\prime} \leq q-2} 2^{q^{\prime}\left(\frac{2}{r}-2\right)}\left\|\Delta_{q^{\prime}} \nabla b\right\|_{L_{t}^{r}\left(L^{\infty}\right)} 2^{\left(q-q^{\prime}\right)\left(\frac{2}{r}-2\right)} .
\end{aligned}
$$

Sachant que $\frac{2}{r}-2<0$, on en déduit alors que

$$
\begin{aligned}
\sup _{q \geq-1} \int_{0}^{t} 2^{-q(1+\varepsilon)-\alpha_{q}(\tau)} & \left\|\Delta_{q} T_{\partial_{j} b} a^{j}\right\|_{L^{2}} d \tau \\
& \lesssim\|\nabla b\|_{\widetilde{L}_{t}^{r}\left(B_{\infty, \infty}^{\frac{2}{r}-2}\right)} \sup _{q \geq-1}\left\|2^{q\left(1-\frac{2}{r}-\varepsilon\right)-\alpha_{q}}\right\| \Delta_{q} a\left\|_{L^{2}}\right\|_{L_{t}^{r^{\prime}}} .
\end{aligned}
$$

De même, pour estimer le deuxième terme de (57), on peut procéder comme si l'on avait $\Delta_{q}\left(T_{a^{j}} \partial_{j} b\right)=S_{q-1} a^{j} \Delta_{q} \partial_{j} b$. On a pour tout $0 \leq t \leq T$,

$$
\begin{aligned}
& 2^{-q(1+\varepsilon)-\alpha_{q}(\tau)}\left\|S_{q-1} a^{j} \Delta_{q} \partial_{j} b\right\|_{L^{2}} \\
& \quad \leq 2^{q\left(\frac{2}{r}-1\right)}\left\|\Delta_{q} \nabla b\right\|_{L^{2}} \sum_{q^{\prime} \leq q-2} 2^{-q^{\prime}\left(\frac{2}{r}+\varepsilon\right)-\alpha_{q}(\tau)}\left\|\Delta_{q^{\prime}} a\right\|_{L^{\infty}} 2^{\left(q^{\prime}-q\right)\left(\varepsilon+\frac{2}{r}\right)} .
\end{aligned}
$$

Sachant que $\alpha_{q} \geq \alpha_{q^{\prime}}$ pour $q \geq q^{\prime}$, on en déduit que

$$
\begin{aligned}
& \int_{0}^{t} 2^{-q(1+\varepsilon)-\alpha_{q}(\tau)}\left\|S_{q-1} a^{j} \Delta_{q} \partial_{j} b\right\|_{L^{2}} d \tau \\
& \quad \leq 2^{q\left(\frac{2}{r}-1\right)}\left\|\Delta_{q} \nabla b\right\|_{L_{t}^{r}\left(L^{2}\right)} \sum_{q^{\prime} \leq q-2}\left\|2^{-q^{\prime}\left(\frac{2}{r}+\varepsilon\right)-\alpha_{q^{\prime}}}\right\| \Delta_{q^{\prime}} a\left\|_{L^{\infty}}\right\|_{L_{t}^{r^{\prime}}} 2^{\left(q^{\prime}-q\right)\left(\varepsilon+\frac{2}{r}\right)} .
\end{aligned}
$$

TOME $136-2008-\mathrm{N}^{\mathrm{O}} 2$ 
Grâce à l'inégalité de Bernstein, on peut donc conclure que

$$
\begin{aligned}
\sup _{q \geq-1} \int_{0}^{t} 2^{-q(1+\varepsilon)-\alpha_{q}(\tau)}\left\|S_{q-1} a^{j} \Delta_{q} \partial_{j} b\right\|_{L^{2}} d \tau & \\
& \lesssim\|\nabla b\|_{\widetilde{L}_{t}^{r}\left(B_{2, \infty}^{\frac{2}{r}-1}\right)} \sup _{q \geq-1}\left\|2^{q\left(1-\frac{2}{r}-\varepsilon\right)-\alpha_{q}}\right\| \Delta_{q} a\left\|_{L^{2}}\right\|_{L_{t}^{r^{\prime}}} .
\end{aligned}
$$

Enfin, sachant que

$$
\Delta_{q} \partial_{j} R\left(a^{j}, b\right)=\sum_{q^{\prime} \geq q-3} \partial_{j} \Delta_{q}\left(\Delta_{q^{\prime}} a^{j} \widetilde{\Delta}_{q^{\prime}} b\right) \quad \text { avec } \quad \widetilde{\Delta}_{q^{\prime}}=\Delta_{q^{\prime}-1}+\Delta_{q^{\prime}}+\Delta_{q^{\prime}+1},
$$

et que $\mathcal{F}\left(\partial_{j} \Delta_{q}\left(\Delta_{q^{\prime}} a^{j} \widetilde{\Delta}_{q^{\prime}} b\right)\right)$ est supporté dans une boule de taille $2^{q}$, on a en vertu de l'inégalité de Bernstein,

$$
2^{-q(1+\varepsilon)-\alpha_{q}(\tau)}\left\|\Delta_{q} \partial_{j} R\left(a^{j}, b\right)\right\|_{L^{2}} \lesssim \sum_{q^{\prime} \geq q-3} 2^{q(1-\varepsilon)-\alpha_{q}(\tau)}\left\|\Delta_{q^{\prime}} a\right\|_{L^{2}}\left\|\widetilde{\Delta}_{q^{\prime}} b\right\|_{L^{2}},
$$

d'où,

$$
\begin{aligned}
& \int_{0}^{t} 2^{-q(1+\varepsilon)-\alpha_{q}(\tau)}\left\|\Delta_{q} \partial_{j} R\left(a^{j}, b\right)\right\|_{L^{2}} d \tau \\
& \lesssim \sum_{q^{\prime} \geq q-3} \int_{0}^{t} 2^{q^{\prime}\left(1-\frac{2}{r}-\varepsilon\right)-\alpha_{q^{\prime}}(\tau)}\left\|\Delta_{q^{\prime}} a\right\|_{L^{2}}\left(2^{\frac{2}{r} q^{\prime}}\left\|\widetilde{\Delta}_{q^{\prime}} b\right\|_{L^{2}}\right) 2^{\left(\alpha_{q^{\prime}}-\alpha_{q}\right)(\tau)} 2^{\left(q-q^{\prime}\right)(1-\varepsilon)} .
\end{aligned}
$$

Pour conclure, l'hypothèse (56) est cruciale. On obtient alors pour tout $q \geq-1$,

$$
\begin{aligned}
\int_{0}^{t} 2^{-q(1+\varepsilon)-\alpha_{q}(\tau)} \| \Delta_{q} \partial_{j} R & \left(a^{j}, b\right) \|_{L^{2}} d \tau \\
& \lesssim\|b\|_{\widetilde{L}_{t}^{r}\left(B_{2, \infty}^{\frac{2}{r}}\right)} \sup _{q \geq-1}\left\|2^{q\left(1-\frac{2}{r}-\varepsilon\right)-\alpha_{q}}\right\| \Delta_{q} a\left\|_{L^{2}}\right\|_{L_{t}^{r^{\prime}}}
\end{aligned}
$$

ce qui, joint à (57), (58) et (59), achève la démonstration.

\section{BIBLIOGRAPHIE}

[1] H. ABIDi \& T. HMidi - «On the global well-posedness for Boussinesq system », J. Differential Equations 233 (2007), p. 199-220.

[2] H. BAhouri \& J.-Y. CHEMIN - «Équations de transport relatives à des champs de vecteurs non-lipschitziens et mécanique des fluides », Arch. Rational Mech. Anal. 127 (1994), p. 159-181.

[3] J.-M. BonY - «Calcul symbolique et propagation des singularités pour les équations aux dérivées partielles non linéaires », Ann. Sci. École Norm. Sup. (4) 14 (1981), p. 209-246. 
[4] M. Cannone, Y. Meyer \& F. Planchon - «Solutions auto-similaires des équations de Navier-Stokes », in Séminaire sur les Équations aux Dérivées Partielles, 1993-1994, exp. no VIII, 12, École Polytech., 1994.

[5] D. CHAE - «Global regularity for the 2D Boussinesq equations with partial viscosity terms », Adv. Math. 203 (2006), p. 497-513.

[6] J.-Y. CHEmin - «Fluides parfaits incompressibles », Astérisque 230.

[7] _ _ « Remarques sur l'existence globale pour le système de NavierStokes incompressible », SIAM J. Math. Anal. 23 (1992), p. 20-28.

[8] _ _ " Théorèmes d'unicité pour le système de Navier-Stokes tridimensionnel », J. Anal. Math. 77 (1999), p. 27-50.

[9] _ _ «e système de Navier-Stokes incompressible soixante dix ans après Jean Leray », in Actes des Journées Mathématiques à la Mémoire de Jean Leray, Sémin. Congr., vol. 9, Soc. Math. France, 2004, p. 99-123.

[10] J.-Y. CHEmin \& N. LERNER - «Flot de champs de vecteurs non lipschitziens et équations de Navier-Stokes », J. Differential Equations 121 (1995), p. 314-328.

[11] D. Córdoba, C. Fefferman \& R. de la Llave - « On squirt singularities in hydrodynamics », SIAM J. Math. Anal. 36 (2004), p. 204-213.

[12] R. DANCHIN - «Density-dependent incompressible viscous fluids in critical spaces », Proc. Roy. Soc. Edinburgh Sect. A 133 (2003), p. 1311-1334.

[13] _ « Estimates in Besov spaces for transport and transport-diffusion equations with almost Lipschitz coefficients », Rev. Mat. Iberoamericana 21 (2005), p. 863-888.

[14] _ _ «On the uniqueness in critical spaces for compressible NavierStokes equations », NoDEA Nonlinear Differential Equations Appl. 12 (2005), p. 111-128.

[15] _ « «niform estimates for transport-diffusion equations », J. Hyperbolic Differ. Equ. 4 (2007), p. 1-17.

[16] W. E \& C.-W. SHU - «Small-scale structures in Boussinesq convection », Phys. Fluids 6 (1994), p. 49-58.

[17] H. Fujita \& T. Kato - «On the Navier-Stokes initial value problem. I », Arch. Rational Mech. Anal. 16 (1964), p. 269-315.

[18] T. HMidi \& S. KeRAANI - « Incompressible viscous flows in borderline Besov spaces », à paraître dans Archive for Rational Mechanics and Analysis.

[19] _ « On the global well-posedness of the two-dimensional Boussinesq system with a zero diffusivity », Adv. Differential Equations 12 (2007), p. $461-480$.

TOME $136-2008-\mathrm{N}^{\mathrm{O}} 2$ 
[20] T. Y. Hou \& C. LI - « Global well-posedness of the viscous Boussinesq equations », Discrete Contin. Dyn. Syst. 12 (2005), p. 1-12.

[21] T. KATO - «Strong $L^{p}$-solutions of the Navier-Stokes equation in $\mathbb{R}^{m}$, with applications to weak solutions », Math. Z. 187 (1984), p. 471-480.

[22] P. G. LemariÉ-Rieusset - Recent developments in the Navier-Stokes problem, Chapman \& Hall/CRC Research Notes in Mathematics, vol. 431, Chapman \& Hall/CRC, 2002.

[23] J. LerAY - «Essai sur le mouvement d'un liquide visqueux emplissant l'espace », Journal de Mathématiques Pures et Appliquées 13 (1934), p. 331418.

[24] _ « Sur le mouvement d'un liquide visqueux emplissant l'espace », Acta Math. 63 (1934), p. 193-248.

[25] J.-L. Lions \& G. Prodi - « Un théorème d'existence et unicité dans les équations de Navier-Stokes en dimension $2 », C$. R. Acad. Sci. Paris 248 (1959), p. 3519-3521.

[26] Y. MeYER - «Wavelets, paraproducts, and Navier-Stokes equations », in Current developments in mathematics, 1996 (Cambridge, MA), Int. Press, Boston, MA, 1997, p. 105-212.

[27] J. Pedlosky - Geophysical fluid dynamics, Springer, 1987.

[28] T. Runst \& W. SickeL - Sobolev spaces of fractional order, Nemytskij operators, and nonlinear partial differential equations, de Gruyter Series in Nonlinear Analysis and Applications, vol. 3, Walter de Gruyter \& Co., 1996.

[29] R. SALmon - Lectures on geophysical fluid dynamics, Oxford University Press, 1998.

[30] O. Sawada \& Y. TANiUchi - « On the Boussinesq flow with nondecaying initial data », Funkcial. Ekvac. 47 (2004), p. 225-250.

[31] M. Vishik - « Hydrodynamics in Besov spaces », Arch. Ration. Mech. Anal. 145 (1998), p. 197-214. 